\title{
Source Localization using a Directional Gamma Ray Spectrometer
}

\author{
by
}

\section{Muhammad Shoaib Khan}

\author{
A Thesis submitted to \\ the Faculty of Graduate and Postdoctoral Affairs \\ in partial fulfillment of \\ the requirements for the degree of \\ Master of Science \\ in
}

Physics

Ottawa Carleton Institute for Physics

Department of Physics

Carleton University

Ottawa, Ontario, Canada

Nov 2012

Copyright @2012 - Muhammad Shoaib Khan 
Library and Archives

Canada

Published Heritage

Branch

395 Wellington Street

Ottawa ON K1A ON4

Canada
Bibliothèque et

Archives Canada

Direction du

Patrimoine de l'édition

395 , rue Wellington

Ottawa ON K1A ON4

Canada
Your file Votre référence

ISBN: 978-0-494-94315-1

Our file Notre référence

ISBN: 978-0-494-94315-1
NOTICE:

The author has granted a nonexclusive license allowing Library and Archives Canada to reproduce, publish, archive, preserve, conserve, communicate to the public by telecommunication or on the Internet, loan, distrbute and sell theses worldwide, for commercial or noncommercial purposes, in microform, paper, electronic and/or any other formats.

The author retains copyright ownership and moral rights in this thesis. Neither the thesis nor substantial extracts from it may be printed or otherwise reproduced without the author's permission.
AVIS:

L'auteur a accordé une licence non exclusive permettant à la Bibliothèque et Archives Canada de reproduire, publier, archiver, sauvegarder, conserver, transmettre au public par télécommunication ou par l'Internet, prêter, distribuer et vendre des thèses partout dans le monde, à des fins commerciales ou autres, sur support microforme, papier, électronique et/ou autres formats.

L'auteur conserve la propriété du droit d'auteur et des droits moraux qui protege cette thèse. $\mathrm{Ni}$ la thèse ni des extraits substantiels de celle-ci ne doivent être imprimés ou autrement reproduits sans son autorisation.
In compliance with the Canadian Privacy Act some supporting forms may have been removed from this thesis.

While these forms may be included in the document page count, their removal does not represent any loss of content from the thesis.
Conformément à la loi canadienne sur la protection de la vie privée, quelques formulaires secondaires ont été enlevés de cette thèse.

Bien que ces formulaires aient inclus dans la pagination, il n'y aura aucun contenu manquant. 


\section{Abstract}

Orphan radioactive sources pose a threat to safety and security and are a concern for various government institutions and the security agencies. It is becoming important to develop robust techniques to find and localise such sources. In the present work, two complementary methods to localize a source have been developed using a directional gamma survey spectrometer. The instrument used consists of four $\mathrm{NaI}(\mathrm{Tl})$ detectors oriented vertically in such a way that the crystals on one side shield the crystals on the other side of this arrangement.

In the gross count method, the total counts from all four detectors were recorded and a fit was performed to reconstruct the source positions based on total counts versus position. For near sources (less than $15 \mathrm{~m}$ ), the accuracy of this method is up to $1 \mathrm{~m}$ in the position along the road and in the distance from the road. For farther sources (from $22 \mathrm{~m}$ to $32 \mathrm{~m}$ ), it provides accuracy up to $10 \mathrm{~m}$ on both.

In the directional method, the relative counts in each crystal as a function of position can be used to measure the angle to the source by forming directional vectors. The survey then returns a field of these vectors, which may be fit to reconstruct the coordinates of the source position. For near sources (less than $15 \mathrm{~m}$ ), this method gives an accuracy of up to $6 \mathrm{~m}$ in position along the road and $4 \mathrm{~m}$ in the distance from the road. For farther sources (from $22 \mathrm{~m}$ to $32 \mathrm{~m}$ ), the accuracy in the position along the road is up to $5 \mathrm{~m}$ and in the distance from the road reduces up to $25 \mathrm{~m}$.

The gross count method provides more accurate and reliable source localization, 
but it does not provide directional information in real time. For this reason. the directional method is used to provide a direction to the source. Multiple truck-borne surveys were conducted using this instrument driving past Na-22 and Cs-137 sources at speeds of $20 \mathrm{~km} / \mathrm{h}$ and $40 \mathrm{~km} / \mathrm{h}$. The surveys were repeated with the sources placed at different distances from the road. Here, we present the results of these measurements. 


\section{Acknowledgments}

I am really thankful to my supervisor, Laurel Sinclair. This work would not have been possible without her kind collaboration and help at every stage of the project. She bore with my mistakes patiently and was always eager to help me make them right, for which I am grateful. Working with her was definitely an inspirational experience for me. This experience will help immensely in my future endeavors. I would also like to thank Henry Seyward, John Carson (NRCan team) and Patrick Saull (NRC) who have contributed to the experimental setup and for their kind gestures from time to time. The funding for this project was provided by Canada's Centre for Security Science.

I am thankful to my family as well, specially my brother Uzair Khan, who has given me unconditional support throughout my educational career. 


\section{Table of Contents}

Abstract $\quad$ ii

Acknowledgments $\quad$ iv

Table of Contents $\quad$ v

List of Tables $\quad$ viii

List of Figures $\quad$ ix

List of Symbols and Acronyms xii

1 Introduction 1

1.1 Aim of present work ....................... 2

1.2 Overview of thesis structure . . . . . . . . . . . . . 3

2 Theory 4

2.1 Radioactivity ........................ 4

2.2 Interaction of radiation . . . . . . . . . . . . . . 6

2.3 Isotopes used in this study . . . . . . . . . . . . . . 8

$2.3 .1 \mathrm{Cs}-137 \ldots \ldots \ldots \ldots$

$2.3 .2 \mathrm{Na}-22 \ldots \ldots \ldots \ldots \ldots$

2.4 Natural radioactivity . . . . . . . . . . . . . . . . 9 
2.5 Count rate........................... 10

2.5 .1 Attenuation ..................... 10

2.5 .2 Inverse square law ................. 11

2.5.3 General expression for count rate . . . . . . . . . . . 12

2.6 Geographic Coordinates . . . . . . . . . . . . . . . 12

2.6.1 Earth-Centered Earth-Fixed coordinates . . . . . . . . . 13

2.6 .2 Universal Transverse Mercator . . . . . . . . . . . . 13

2.7 GeographicLib Package . . . . . . . . . . . . . . . 15

3 Instrumentation $\quad 16$

3.1 Scintillator detector . . . . . . . . . . . . . . 16

3.2 Sodium Iodide $(\mathrm{NaI}(\mathrm{Tl})) \ldots \ldots \ldots \ldots$

3.3 Efficiency . . . . . . . . . . . . . . . 19

3.4 Our Instrument . . . . . . . . . . . . . 20

4 Methods $\quad 22$

4.1 Previous work ....................... 22

4.1.1 Source detection ................... 22

4.1 .2 Source localization . . . . . . . . . . . . 23

4.2 Experimental setup . . . . . . . . . . . . . . 25

4.3 Gross Count method $(\mathrm{GC}) \ldots \ldots \ldots . \ldots 26$

4.4 Directional Method $(\mathrm{DM}) \ldots \ldots \ldots \ldots$

4.4.1 Derivation of directional vectors for limiting cases . . . . . . 31

4.4 .2 Directional calibration . . . . . . . . . . . 33

4.4 .3 Probable source location . . . . . . . . . . . . . 38

5 Results and Analysis $\quad 42$

5.1 Energy spectra for source identification . . . . . . . . . . . 42 
5.1.1 Background energy spectrum .............. 42

5.1 .2 Na-22 energy spectrum . . . . . . . . . . . . . 43

5.1.3 Cs-137 energy spectrum . . . . . . . . . . . . . 45

5.2 DGPS correction ....................... 45

5.3 Lag correction . . . . . . . . . . . . . . . . . 48

5.4 Gross count method $(\mathrm{GC})$. . . . . . . . . . . . . 49

5.4.1 Gross count plots for Na-22 . . . . . . . . . . 49

5.4 .2 Gross count plots for $\mathrm{Cs}-137 \ldots \ldots . . \ldots 56$

5.5 Directional method $(\mathrm{DM}) \ldots \ldots \ldots \ldots$

5.5 .1 Directional method plots for $\mathrm{Na}-22 \ldots \ldots . . \ldots 59$

5.5.2 Directional method plots for Cs-137 . . . . . . . . . . 70

5.6 Angular resolution measure . . . . . . . . . . . 71

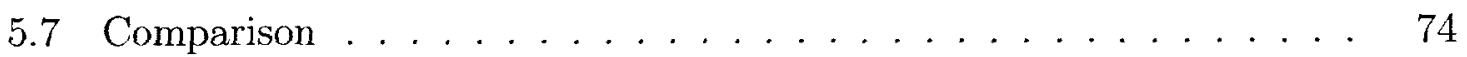

$\begin{array}{lll}6 & \text { Discussion and conclusions } & 75\end{array}$

6.1 Gross count method ................. 75

6.2 Directional Method . . . . . . . . . . . . . . . 77

6.3 Conclusion . . . . . . . . . . . . . . . . . 78

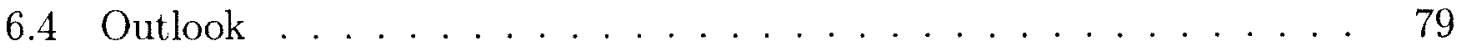

$\begin{array}{ll}\text { Appendix A Gross Count Method } & 81\end{array}$

$\begin{array}{ll}\text { Appendix B Directional Method } & 86\end{array}$

$\begin{array}{ll}\text { References } & 90\end{array}$ 


\section{List of Tables}

2 For Na-22: at $20 \mathrm{~km} / \mathrm{h}$, the mean of the fit extracted results over sixteen passes for $\mathrm{N}, \mathrm{x}_{0}$ and $D_{0}$ with RMS deviations. . . . . . 55

3 For Na-22: at $40 \mathrm{~km} / \mathrm{h}$, the mean of the fit extracted results over sixteen passes for $\mathrm{N}, \mathrm{x}_{0}$ and $D_{0}$ with RMS deviations. . . . . .

4 For Cs-137: at $20 \mathrm{~km} / \mathrm{h}$, the mean of the fit extracted results over 16

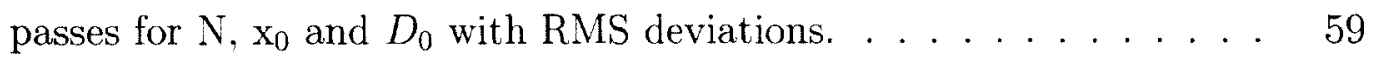

5 For $\mathrm{Na}-22$, at $20 \mathrm{~km} / \mathrm{h}$, the means of the measured values of $\mathrm{x}_{0}, D_{0}$ with RMS deviations. . . . . . . . . . . . . . . . . 67 


\section{List of Figures}

1 Decay scheme of Cs-137 with branching ratio. . . . . . . . . 8

2 Decay mechanisms of $\mathrm{Na}-22$ with branching ratio. . . . . . . . . 9

3 Gamma rays are attenuated after passing through detector. . . . . . . 11

4 Radiation intensity is reduced four times when distance is doubled. . 12

5 Universal Transverse Mercator zones for locating a position on earth . 14

6 Scintillating photons are converted to photoelectrons in PMT. . . . . 17

7 Activator induced additional excited energy levels in forbidden band . 18

8 The one of four detectors used and additional components. . . . . . . 21

9 Satellite image of the experiment's location with schematics of setup. 26

10 Schematics for gross count method. . . . . . . . . . . . 27

11 The count rate dependence on distance from the road is demonstrated. 28

12 The detectors with assigned letters representing respective counts in each detector and the directional vector pointing towards the source is shown . . . . . . . . . . . . . . . . . . 30

13 The angle $\Psi$; the sum of the angles $\Phi$ and $\theta^{\prime} \ldots \ldots \ldots 31$

14 Derivation of directional vector; case $1 \ldots \ldots \ldots . \ldots 32$

15 Derivation of directional vector; case $2 \ldots \ldots \ldots \ldots$

16 The correlation between the true angle and measured angle in directional calibration of the detector . . . . . . . . . 34

17 Angular resolution plots for four angles . . . . . . . . . 35 
18 Directional calibration of detector system plot . . . . . . . . . 36

19 Directional calibration of detector system plot . . . . . . . . 37

20 Angular resolution plots for four angles . . . . . . . . . . . . 38

21 Northing versus the position along the road (Easting) is shown. The optimum source location is pointed by arrows. . . . . . . . . . . . 39

22 Selecting a point $\mathrm{P}$ in probable source region and drawing lines . . . . 40

23 The distance function developed as a function of position as a surface 41

24 Measured energy spectrum and averaged total counts for background (in energy window $450-550 \mathrm{keV}$ ) due to natural radionuclides. . . . . 43

25 Energy spectrum for identification of $\mathrm{Na}-22 \ldots \ldots . \ldots 44$

26 Energy spectrum for identification of Cs-137 . . . . . . . . . . 45

27 DGPS correction of the measured data. . . . . . . . . . . . . . 47

28 Schematics of typical spectra demonstrating the lag effect. The measured spectrum should actually be attributed to position 'a' marked in the figure. . . . . . . . . . . . . . . 48

29 A demonstration of lag correction applied. . . . . . . . . . . 49

30 The counts profile with a fit for $\mathrm{Na}-22$, for all positions, at $20 \mathrm{~km} / \mathrm{h}$, in gross count method. . . . . . . . . . . . . . . 51

31 GC for Na-22: reconstructed source positions for 16 passes at 20 and $40 \mathrm{~km} / \mathrm{h} \ldots \ldots \ldots \ldots \ldots \ldots$

32 GC for $\mathrm{Na}-22$ : the mean of reconstructed source positions at $20 \mathrm{~km} / \mathrm{h}$. 53

$33 \mathrm{GC}$ for $\mathrm{Na}-22$ : the mean of reconstructed source positions at $40 \mathrm{~km} / \mathrm{h}$. 54

34 Gross counts in energy window $550 \mathrm{keV}$ to $710 \mathrm{keV}$ for Cs-137 for all positions A-C. . . . . . . . . . . . . . . . . 56

35 GC for Cs-137: the mean of reconstructed source positions, A-C. . . . 58

36 The measure of significance for all source positions up to D . . . . . 61 
37 DM for Na-22: at $20 \mathrm{~km} / \mathrm{h}$. the direction arrows for one pass versus position along the road . . . . . . . . . . . . . . 62

$38 \mathrm{DM}$ for $\mathrm{Na}-22$ : at $20 \mathrm{~km} / \mathrm{h}$, the distance function $\mathrm{D}(\mathrm{x}, \mathrm{y})$ versus Easting and Northing. . . . . . . . . . . . . 63

39 DM for Na-22: measured reconstructed source positions for 16 passes. 64

40 DM for Na-22: the mean of reconstructed source positions. . . . . . . 65

41 A demonstration of the error on position along the road and perpendicular from the road. . . . . . . . . . . . . . 66

$42 \mathrm{DM}$ for $\mathrm{Na}-22$ : at $40 \mathrm{~km} / \mathrm{h}$, the direction arrows for one pass versus position along the road . . . . . . . . . . . . . . 68

$43 \mathrm{DM}$ for $\mathrm{Na}-22$ : at $40 \mathrm{~km} / \mathrm{h}$, the distance function $\mathrm{D}(\mathrm{x}, \mathrm{y})$ versus Easting and Northing. . . . . . . . . . . . . . 69

44 The significance plot for the $\mathrm{Cs}-137$ source, at positions $\mathrm{A}$ and B . . 70

45 DM for Cs-137: reconstructed source positions for 16 passes. . . . . . 71

46 The schematic of angular distribution and difference of measured angle with the true source position. . . . . . . . . . . . . . 72

47 The spread of measured arrows and angles to the true source fitted with Gaussian. . . . . . . . . . . . . . . . . 


\section{List of Symbols and Acronyms}

\begin{tabular}{ll} 
Acronyms and Symbols & Definition \\
\hline $\mathrm{BGO}$ & bismuth germanate \\
$\mathrm{Bq}$ & Becquerel \\
$\mathrm{CsI}$ & cesium iodide \\
$\mathrm{Tl}$ & thallium \\
$\mathrm{NaI}$ & sodium iodide \\
$\mathrm{NaI}(\mathrm{Tl})$ & sodium iodide - thallium doped \\
$\mathrm{K}$ & potassium \\
$R$ & variable distance from truck's position to the source \\
$\mathrm{DM}$ & directional method \\
$\mathrm{FWH}$ & count rate \\
& full-width at half-maximum count method \\
&
\end{tabular}




$\begin{array}{ll}I & \text { intensity } \\ S & \text { source strength } \\ \text { detector area } & \text { position along the road } \\ \mathrm{x}_{0} & \text { shortest distance from the road } \\ D_{0} & \text { root mean square } \\ \text { RMS } & \text { RMS - for position along the road (Easting) } \\ \text { RMS } & \text { RMS - for distance from the road (Northing) } \\ \text { RMS } & \text { linear attenuation coefficient } \\ \mu & \text { linear attenuation coefficient of air } \\ D_{0} & \text { significance } \\ \mu_{\text {air }} & \end{array}$




\section{Chapter 1}

\section{Introduction}

The importance of research in radiological surveys is highlighted by nuclear disasters such as that in Fukushima, Japan (2011) or Chernobyl, Ukraine (1986). Such calamities remind us of the need to develop new and robust techniques to localize sources of hazardous radiation and radioactive materials.

Within the past few decades, radioactive sources have been lost, dumped irresponsibly or transferred illegally. A commonly used name for this type of source is orphaned source. Orphaned sources are those that pose a significant enough radiological hazard to warrant regulatory control but which are not under any regulatory control. Orphan sources pose a serious problem [1].

Useful techniques using mobile gamma surveys have been developed by researchers to deal with radiological hazards. In mobile gamma surveys there are two common survey types that are widely used: air-borne gamma spectrometry and car-borne gamma spectrometry ( [2], [3], [4]). Air-borne surveys have many

advantages, such as covering a large area in a short amount of time. Car-borne surveys, however, do not require elaborate instrumentation and planning and are more economical than air-borne, as no aircraft or airports are required.

These techniques are useful for both urban and rural settings. Under normal circumstances, the urban areas in particular are characterized by a wide variety 
of gamma radiation spectra and very fast changes in the ever-present background radiation. Consideration to the background must also be included in any analysis work. In this work, the car-borne survey technique was used.

The experimental set up was done by Laurel Sinclair, John Carson, Henry Seyward (NRCan), and Patrick Saull (NRC) who provided sources. The tracks of the truck and positions of the well placed sources were surveyed using a conventional total station (theodolyte and laser range finder). Once it was set up, the NRCan team performed the experiment. Before starting the experiment with sources, background runs were performed and recorded data were saved on the instrument's laptop. The truck moved back and forth on a given track of $\sim 200 \mathrm{~m}$. It was made to pass by well placed gamma emitters 16 times and at each pass, the counts (signals) from gamma radiation were recorded, along with the GPS position of the truck.

\subsection{Aim of present work}

The purpose of this work is to develop and evaluate methods to localize radioactive point sources. The detector instrument $(\mathrm{NaI}(\mathrm{Tl}))$ was placed in a truck and driven past gamma emitters at different distances from the road. The survey data were post-processed using the methods described in this work. The methods are to be applied where the strength and identity of the source are not a priori known.

The sources were placed at four different distances (up to $32 \mathrm{~m}$ ) from the road and the truck was driven past these sources at speeds of $20 \mathrm{~km} / \mathrm{h}$ and $40 \mathrm{~km} / \mathrm{h}$. The source positions were reconstructed during post-processing of the recorded data. Two offline methods (the gross count method and directional method) were developed and used to reconstruct source positions.

The gross count method is a widely used technique in the literature $([5],[6],[7])$ for source localization. In the gross count method, the total photopeak counts were 
fitted to a known expression to recontruct the source positions at different distances from the road. The results of the fit provided us with the source location. This method does not determine the side of the road where the source is located.

In order to determine the side of the road where the source is located, the directional method has to be employed. In the directional method, the relative counts recorded by the gamma spectrometer are used to form directional vectors for a selected energy window to reconstruct the direction to the source as a function of the position of the truck along the road. The direction to the source was given by arrows. The probable source location was given by the minimum of the surface which represents the sum of the squares of the distances of the arrows from each point in space. The minima of the surface plots, providing the optimum source location, were extracted using Minuit [5], in a data analysis package. This method is advantgeous by also providing the direction to the source location in real time.

A detailed analysis was performed and reconstructed source positions were compared to the true source positions for both methods.

This work was accepted and presented at SORMA WEST 2012 (IEEE Symposium on Radiation Measurements and Applications), in Oakland, California, USA [8].

\subsection{Overview of thesis structure}

The present work consists of six chapters in total. Chapter 2 deals with the theory and basic concepts and Chapter 3 gives a brief description of the scintillator detector of the type $\mathrm{NaI}(\mathrm{Tl})$ and the instrument used. The methods developed in the present work are given a detailed description in Chapter 4 . Chapter 5 gives the detailed analysis of the results. Finally, Chapter 6 provides a discussion and concludes the present work. The appendices (A and B) after Chapter 6 contain the most important functions and plotting routines only. The list of references is provided at the end. 


\section{Chapter 2}

\section{Theory}

When it comes to source localization, numerous factors must be considered including radioactive decay processes, interaction of radiation with matter, the detector's efficiency, and the geographic coordinate system used for positions. A brief description of the radiation mechanisms and coordinate systems used for localization of point sources, is given in this chapter.

\section{$2.1 \quad$ Radioactivity}

The spontaneous disintegration of nuclei is referred to as radioactivity. It is a phenomenon in which nuclear particles are emitted from unstable atomic nuclei. The strength of radiation sources is generally described by their activity. The activity of a radioactive source is defined by its rate of decay,

$$
\frac{\mathrm{dN}}{\mathrm{dt}}=-\lambda \mathrm{N}
$$

where $\mathrm{N}$ is the number of radioactive nuclei and $\lambda$ is the decay constant.

This equation represents the decay-rate only. The net value of the decay is given by the decay rate multiplied by the fraction of such decays that led to the decay 
product [9]. The fraction of decays leading to the decay product is called the branching ratio.

The System International (SI) unit of activity is the Becquerel $(\mathrm{Bq})$. One $\mathrm{Bq}$ is the quantity in which one nucleus decays per second (i.e., 1 disintegration/second). An older non-SI unit of activity is the Curie $(\mathrm{Ci})$ which is defined as $3.7 \times 10^{10} \mathrm{~Bq}$. Generally, smaller units like mCi (millicurie) are used for practical radioactive sources.

The proportion of neutrons to protons determines whether a nucleus is stable or not. There are many reference charts available to find out about stable and unstable nuclei [10]. Most nuclear decay processes result in gamma emission. In many cases, before the emission of gamma radiation, the nucleus decays to a daughter nucleus with the emission of a particle and then decays to the ground state after emitting gamma radiation. The sources used in the present work undergo beta decay.

\section{Beta minus decay}

In beta minus $\left(\beta^{-}\right)$decay a negative electron is emitted along with an anti-neutrino thus leaving behind a daughter nucleus having one smaller atomic number (Z) and the same mass number (A). It converts a neutron into a proton and an electron and anti-neutrino are emitted. Many elements decay through the beta minus process. It is generalised by,

$$
{ }_{Z}^{A} \mathrm{X} \longrightarrow{ }_{Z+1}^{A} \mathrm{Y}+\beta^{-}+\bar{\nu}
$$

For example, Cs-137 undergoes beta minus decay given by,

$$
{ }_{55}^{137} \mathrm{Cs} \longrightarrow{ }_{56}^{137} \mathrm{Ba}+\beta^{-}+\bar{\nu}
$$




\section{Beta plus decay}

Beta plus $\left(\beta^{+}\right)$decay occurs when a parent nucleus emits a positron along with a neutrino, leaving behind a daughter nucleus having one lower atomic number but the same mass number. This happens when the binding energy of the parent nucleus is less than the daughter nucleus. It is this energy difference that causes a proton to change into a neutron, positron, neutrino and into the kinetic energy of these particles. It is generalised by.

$$
{ }_{Z}^{A} \mathrm{X} \longrightarrow{ }_{Z-1}^{A} \mathrm{Y}+\beta^{+}+\nu .
$$

For example, Na-22 decays through a positron $90 \%$ of the time, as given by,

$$
{ }_{11}^{22} \mathrm{Na} \longrightarrow{ }_{10}^{22} \mathrm{Ne}+\beta^{+}+\nu .
$$

\subsection{Interaction of radiation}

Gamma rays are detected when they interact with the material of a detector. As the gamma radiation passes through material, it interacts mainly in three ways: photoelectric absorption, Compton scattering and pair production.

In photoelectric absorption, a gamma photon interacts with a bound electron of the absorber or detector material and loses all of its energy and ceases to exist. Photoelectric absorption is important in gamma ray spectrometry because the gamma gives up all of its energy, thus giving a full energy peak. In $\mathrm{NaI}(\mathrm{Tl})$, the chance of photoelectric absorption is greater than $50 \%$ of the cross section at $200 \mathrm{keV}$ and becomes much less important at higher energies ( $6 \%$ at $1 \mathrm{MeV})$.

In Compton scattering, the incident gamma radiation loses enough energy to an atomic (recoil) electron to cause its ejection and the scattered gamma leaves with a 
lower energy and at an angle to its original direction. In $\mathrm{NaI}(\mathrm{Tl})$. Compton scattering is the principal absorption mode (greater than $50 \%$ of the cross section) for gamma rays in the energy range from $260 \mathrm{keV}$ to just below $6.8 \mathrm{MeV}$ [11]. When Compton scattering takes place in a detector, the detector medium usually stops the electron and thus an output pulse is produced which is proportional to the energy lost by the incident gamma ray. The probability of interaction depends on electron density and that is proportional to $\mathrm{Z} / \mathrm{A}$ which is nearly constant for all materials [12].

When the energies of the gamma rays exceed $1.02 \mathrm{MeV}$, the pair production phenomenon becomes possible. It takes an important place as an absorption mechanism particularly at energies exceeding $5 \mathrm{MeV}$. In this process, in the Coulomb field of a nucleus, the energy of the incident gamma is converted into the mass of the positron-electron pair. Any incident energy exceeding the mass of the two particles (1.02 MeV) appears as the kinetic energy of the pair and also as the (nearly negligible) recoil energy of the nucleus. The positron can annihilate during flight or when it reaches the end of its range, combine with a free electron and annhiliate. The entire mass of these two particles is then converted into two gammas of $511 \mathrm{keV}$ each, released in the opposite direction to each other.

For the gamma sources used, Cs-137 (662 keV) and Na-22 (using the $511 \mathrm{keV}$ peak), the full energy peak is predominantly formed by those interactions where the gamma loses its energy through a series of Compton scatterings followed by a photoelectric absorption. Those events that fall below the full peak in the spectrum are those where the gamma ray loses only part of its energy inside the detector, and then escapes.

In any of these three processes, the secondary electrons or positron would frequently have enough energy to produce ionization themselves. Knowledge of these effects is necessary in order to interpret a measured gamma spectrum and identify isotopes in it. 


\subsection{Isotopes used in this study}

\subsubsection{Cs-137}

The Cs-137 energy level schematic, presented in Fig. 1, shows two ways of beta minus decay that it can go through. One way is a highly energetic one ( $\beta^{-}$decay $-5.6 \%$ of the time), going directly to the ground state. The other way is through beta minus decay $(94.4 \%[13]$ or $94.6 \%$ of the time [10]) to the excited state of the daughter element Ba-137, then emitting a gamma of $662 \mathrm{keV}$.

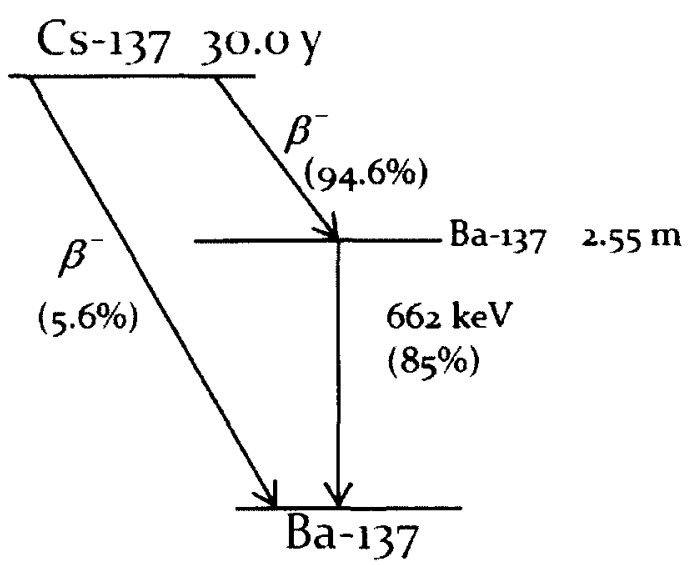

Figure 1: Cs-137 decay scheme, showing daughter energy levels.

The excited state of Ba-137 from the $\beta^{-}$decay, is almost stable and decays with a half-life of $2.55 \mathrm{~min}$. When the half-life of an excited state is long, that state is known as an isomeric state. In this case, the excited state's energy $(0.662 \mathrm{MeV})$ is mostly emitted as a gamma-ray, as shown in Fig. 1.

\subsubsection{Na-22}

Fig. 2 shows the decay scheme of the Na-22 with the daughter energy levels. Na-22 is a well known positron emitter and serves as a high-energy gamma source. The $\mathrm{Na}-22$ source decays $90 \%$ of the time through $\beta^{+}$decay to an excited state of $\mathrm{Ne}-22$ 
[10], giving a positron which in turn gets annihilated with an electron giving off two gammas each with energy equal to the mass of an electron $511 \mathrm{keV}$. These photons are always emitted in opposite direction or 180 degrees to each other to conserve momentum.

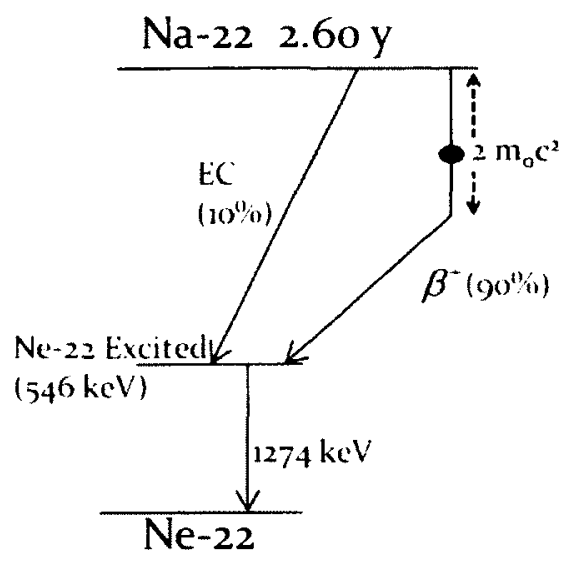

Figure 2: $\mathrm{Na}-22$ decay scheme, showing daughter energy levels.

The resulting Ne-22 daughter de-excites, producing a $1274.5 \mathrm{keV}$ gamma. The gamma-decay energy of $1274.5 \mathrm{keV}$ indicates that the pair production is possible. The Na-22 spectrum exhibits a peak at $1274.5 \mathrm{keV}$, in addition to a sharp $511 \mathrm{keV}$ peak.

\subsection{Natural radioactivity}

Natural radioactivity is abundant and present all the time, caused by naturally occuring radionuclides. The sources of natural radioactivity are mainly the heavier elements and seldom the lighter ones. Some of these sources are found in the earth, mainly in the lithosphere. Others are found in the atmosphere. The ones that are found in the earth are in the form of ores of uranium (U), thorium (Th) (uranium and thorium decay series) and their decay products along with potassium (K) salts. 
Their concentrations in granite are about $4 \mathrm{mg} / \mathrm{kg}$ (for $\mathrm{U}$ ) and $13 \mathrm{mg} / \mathrm{kg}$ (for $\mathrm{Th}$ ). In sea water. the concentration of uranium mineral (pitchblende) is $3 \mu \mathrm{g}$ per litre [14].

\subsection{Count rate}

The activity of a source is the total number of emissions per second. The measured number of counts per second from an isotropic source is less than the actual activity of the source, due to the fact that the detectors usually do not encompass the source and hence only detect gamma photons emitted into a small fraction of the total solid angle surrounding the source.

\subsubsection{Attenuation}

A gamma-ray must interact within the sensitive volume of the detector in order to be seen. In the year 1900, gamma-rays were identified by Becquerel and Villard as a component of the radiation from uranium and radium having much higher penetrability than alpha and beta particles. Then in 1909, Soddy and Russell figured out that gamma ray attenuation followed an exponential law and that the ratio of the attenuation coefficient to the density of the material through which it is attenuated was nearly constant for all materials [15].

Although the radionuclides of $\mathrm{Na}-22$ and Cs-137 emit gamma rays at fixed energies and rates, the gamma-ray intensity measured outside a sample is always attenuated, as shown in Fig. 3, according to,

$$
I=I_{0} \times e^{-\mu L},
$$

where $I_{0}$ is the intensity (count rate per unit area) of the gamma radiation at the location where it is incident on the absorber's surface of thickness $\mathrm{L}, \mu$ is the linear 


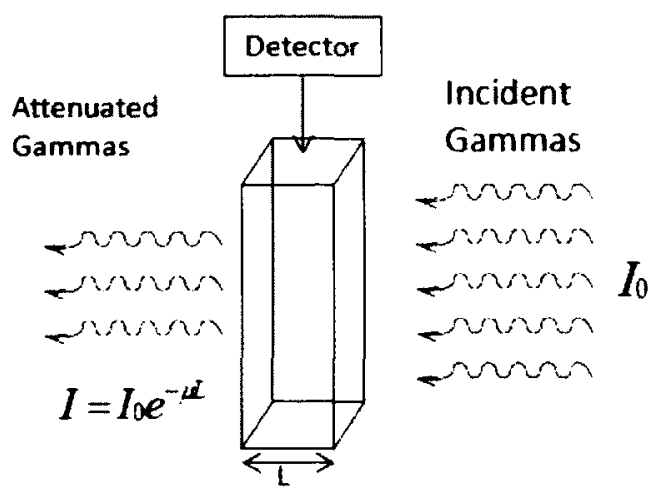

Figure 3: The incident gamma rays on a detector are attenuated after passing through it.

attenuation coefficient and $\mathrm{I}$ is the intensity after it has emerged from the absorber. This is known as the exponential law of gamma attenuation.

\subsubsection{Inverse square law}

An isotropic source radiates uniformly in all directions into a sphere centred at the source. Therefore, the relationship between the intensity $(I)$ of an isotropic source and distance can be characterised by,

$$
I=\frac{S A}{4 \pi R^{2}}
$$

where $S$ is the source strength (number of disintegrations per second), $A$ is the area, and $I$ is the intensity at a distance $R$, ignoring attenuation for now.

The decrease with the inverse square of the distance of the intensity of gamma rays coming from an isotropic source has been experimentally observed [16] and is illustrated in Fig. 4. 


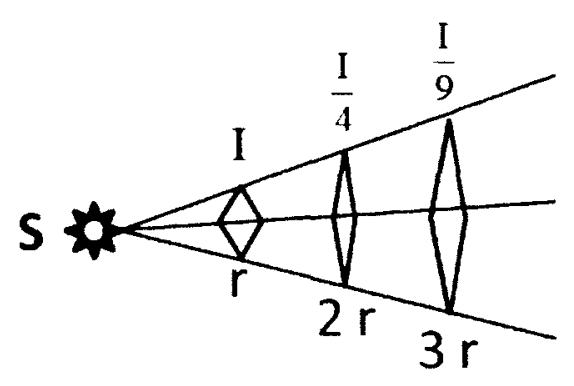

Figure 4: This shows the radiation intensity I decreases as the distance increases. At distance $2 \mathrm{r}$ and $3 \mathrm{r}$, the intensity is $1 / 4$ and $1 / 9$ of the intensity at $\mathrm{r}$, respectively. The figure is not scaled and ignores attenuation.

\subsubsection{General expression for count rate}

The decrease in the count rate in comparison to the actual activity is also affected by the attenuation in the air and inverse square law. When attenuation in the air along with inverse square law is considered, the gross counts received by the detector are given by,

$$
\mathrm{C}=\frac{1}{4 \pi R^{2}} S A \mathrm{e}^{-\mu_{\alpha i r} R},
$$

where $S$ is the emission rate of the source, assuming an efficiency of $100 \%$ for the detector and a branching ratio of $100 \%$ for the isotope, $\mu_{\text {air }}$ (at $662 \mathrm{keV}$ is $\sim 0.00093 \mathrm{~m}^{-1}$ and at $500 \mathrm{keV}$ is $\sim 0.000011 \mathrm{~m}^{-1}$ ) is the linear attenuation coefficient for air, $A$ is the effective detector area and $R$ is the distance from the source to the detector. The gross count method in the present work is based on this principle.

\subsection{Geographic Coordinates}

Geographic coordinates provide a system in which any location on earth can be specified by a set of numbers or letters. These coordinates represent eastward and 
northward positions along with the altitude. The most commonly used geographical coordinates are latitude. longitude and elevation. which is the height above a fixed reference point on the surface of the earth.

\subsubsection{Earth-Centered Earth-Fixed coordinates}

Earth-Centered Earth-Fixed (ECEF) is a Cartesian coordinate system in which the position of a point is described by $x-, y$ - and $z$-axes with the origin located at the center of the mass of the earth. The positive z-axis is the direction through the north pole and the $x y$-plane is the equatorial plane. The $\mathrm{x}$-axis intersects the prime meridian which is known as the zero point of longitude.

These coordinates are used by most satellites to localize a point on earth easily, as only the center of the earth and orientation of the axes is required. The coordinates are generally converted to latitude, longitude and height using an ellipsoid model within a given reference frame, such as the World Geodetic System 84 (WGS84) [17].

\subsubsection{Universal Transverse Mercator}

The Mercator projection is a cylindrical map projection developed by Flemish geographer and cartographer Gerardus Mercator in 1569. The Universal Transverse Mercator (UTM) is a coordinate system that uses two-dimensional Cartesian coordinates to provide a location on the surface of the earth. This system divides the earth into 60 numbered (1 to 60 ) zones with each zone in a six degree band of longitude and uses transverse Mercator projection for each zone. These numbered zones begin counting at 1 from the International Date Line, at 180 west longitude (between Alaska and Russia), to 30 at the Prime Meridian (Greenwich, England), and up to 60 back to the International Date Line. The UTM-coordinates system is valid for areas on earth from $84^{0} \mathrm{~N}$ to $80^{\circ} \mathrm{S}$ latitude [18]. 
In order to locate a point. one must find the UTM zone in which the point is located. Fig. 5 shows different zones over Europe.

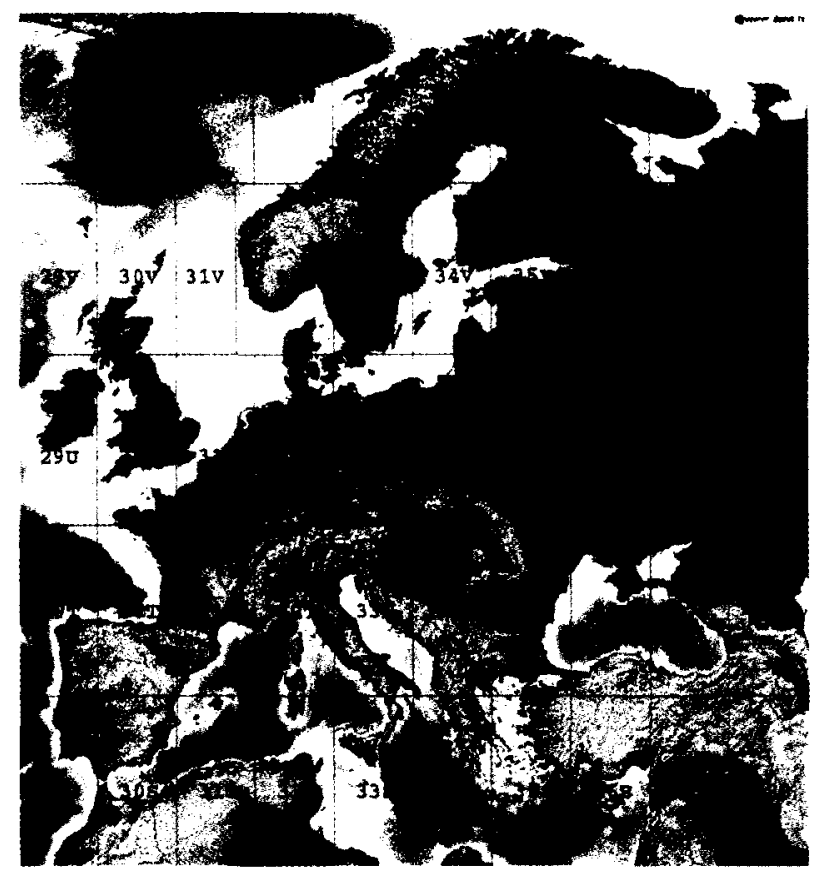

Figure 5: The numbered zones in Universal Transverse Mercator system are shown from $29 \mathrm{~S}$ to $38 \mathrm{~W}$ [19](used under permissions to copy or modify and distribute).

In this system, the eastward distance within each zone is called Easting and ranges from 167,000 meters to 833,000 meters at the equator and the range narrows down towards the poles. In order to avoid negative numbers, each zone's central meridian is set at 500,000 meters East [20]. In any arbitrary zone, if a point has Easting 300,000 meters, it is $200 \mathrm{~km}$ west of the central meridian measured on the transverse Mercator projection.

The northward distance is called northing and is independent of the zone. It ranges from 0 at the equator with the maximum northing at about 9,300,000 meters at the latitude 84 degrees. It forms the north end of the UTM. The northward latitude band is eight degrees wide and assigned a letter from $\mathrm{C}$ to $\mathrm{X}$. 
A location is described by the zone number, for example, $33 \mathrm{~V}$ (see Fig. 5) and associated Easting and Northing pair. The ECEF coordinates in a given data are usually converted to UTM, as it provides a two dimensional or flat system of coordinates.

In order to make the Easting and Northing numbers easier to display, an arbitrary Easting Offset and an arbitrary Northing offset have been subtracted from all Easting and Northing measurements presented in this thesis. This has the effect of bringing the Eastings and Northings into a range between 0 and $300 \mathrm{~m}$. It has the additional desired effect of suppressing the exact location of the experiments.

\subsection{GeographicLib Package}

An analysis can also be affected by how the shape of the earth is approximated. The GPS instrument used in our experiment reported positions in ECEF coordinates. These coordinates were converted to the UTM coordinate system, using the geographiclib package [21].

Geographiclib is based on different algorithms developed by various mathematicians. The geographiclib provides a set of test data as well to check the implementation. These algorithms, presented in the paper published by Charles F. F. Karney, allow the transverse Mercator projection to be computed with high accuracy [21]. 


\section{Chapter 3}

\section{Instrumentation}

\subsection{Scintillator detector}

A substance that produces scintillation photons when subjected to ionizing radiation is known as a scintillator. A scintillator detector or counter is a device to record the counts of incident radiation, particularly gamma radiation. Ideally, these detectors show high counting efficiency (i.e., the ability to convert excitation energy to fluorescent radiation) with high absorption as they can be produced in a large volume [22]. Typical counting rates can be up to $10^{8}$ per second. The light output is proportional to the energy absorbed from radiation. The area of the signal pulse in the end is proportional to the total energy of the scintillating photons, thus an energy spectrum can be measured [23]. 


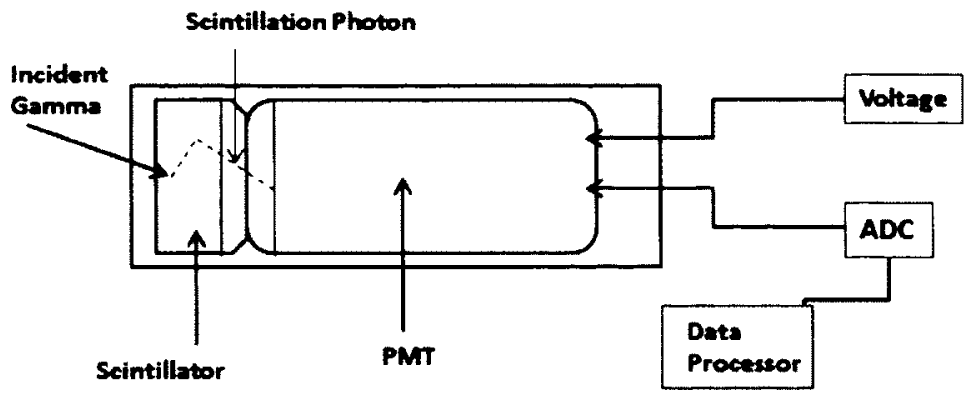

Figure 6: A schematic of a scintillator detector as used in most present day systems.

A scintillator detector is usually comprised of a scintillating crystal, a photomultiplier tube (PMT) and a signal processing unit, as shown in Fig. 6. Incident radiation triggers scintillating photons which are converted into photoelectrons that are multiplied and amplified before their signal is measured.

\section{Types of Scintillators}

There are two types of scintillators: organic and inorganic. The organic scintillators can be in a pure crystalline state, liquid organic solution or plastic form. The commonly used organic crystals are stilbene $\left(\mathrm{C}_{14} \mathrm{H}_{12}\right)$, anthracene $\left(\mathrm{C}_{14} \mathrm{H}_{12}\right)$ and naphthalene $\left(\mathrm{C}_{10} \mathrm{H}_{8}\right)$. The organic scintillators have poor energy resolution, due to a low number of photons produced per keV of energy deposited [9].

The inorganic scintillators are in crystalline (solid) form and the most commonly used are sodium iodide ( $\mathrm{NaI}$ ), CsI (cesium iodide) and $\mathrm{LaBr}_{3}$ (lanthanum bromide).

\section{Activator}

Primary electrons produced by gamma ray interactions lose their energy by creating secondary electron-hole pairs. In materials known as insulators or semiconductors, electrons only have available discrete bands of energy. The valence band is where electrons are bound to the lattice sites. The conduction band is an unfilled band 
where the range of electron energy is higher than that of valence band, allowing electrons to move freely within a crystal. The forbidden band is an intermediate band where the electrons can never be found in a pure crystal [24].

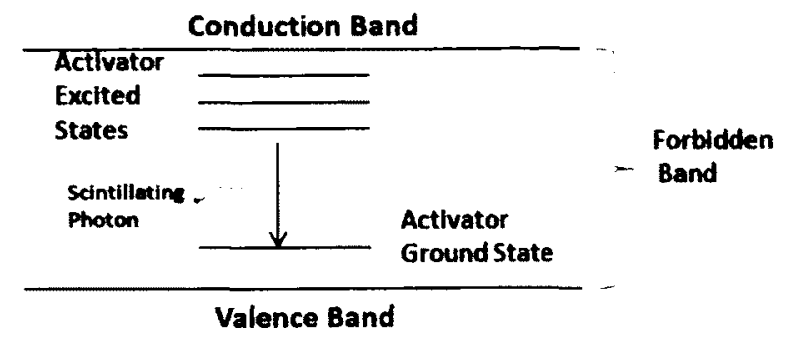

Figure 7: Activator induced additional excited energy levels in the forbidden band in a scintillator crystal.

The mechanism of scintillation in an inorganic scintillator is determined by the crystal lattice of the material used. In these materials, the processes of energy transfer have been described in detail by Murray [25] and Kaufman et al [26].

In some of the inorganic scintillators, the scintillation is inefficient, such as in the case of $\mathrm{NaI}$ and CsI. This is one of the reasons to use a technique called doping. In this technique a small trace impurity of a suitable material is added and energy states are created as a result within the forbidden band, as shown in Fig. 7. This increases the likelihood of an emitted photon lying within the visible range.

\subsection{Sodium Iodide $(\mathrm{NaI}(\mathrm{Tl}))$}

In 1948, Robert Hafstadter showed that sodium iodide (NaI) doped with $\mathrm{Tl}$ produces a large light output when radiation is absorbed [9]. Since this discovery, NaI(Tl) still remains prominent in scintillation spectrometry of gamma radiation, despite research into other scintillators.

Due to its relatively high atomic number $(Z=53)$, the iodine constituent assures 
photoelectric absorption. The high intrinsic efficiency and large photofraction have also contributed to its use in spectrometry. Other inorganic scintillators, such as bismuth germanate $\left(\mathrm{Bi}_{4} \mathrm{Ge}_{3} \mathrm{O}_{12}\right)$ or cesium iodide (CsI), have an even higher density or effective atomic number and therefore the response function gives even greater detection efficiency and photofraction [9].

$\mathrm{NaI}$ is an alkali halide (inorganic) crystalline scintillator to which a trace impurity of thallium iodide is added. $\mathrm{Tl}$ works perfectly for doping NaI crystals. It gives rise to extra levels called activator levels and leads to the emission of a photon serving as the basis for the scintillation process. It is the best activator for both NaI and CsI. For a variety of inorganic materials, it takes three times the band gap energy to create one electron-hole pair. In NaI, this means $20 \mathrm{eV}$ of a charged particle's energy must be deposited in the crystal to create one electron-hole pair. To create $5 \times 10^{4}$ electron-hole pairs, $1 \mathrm{MeV}$ of the particle'e energy deposition is required. Experimental findings have shown that Nal activated with $\mathrm{Tl}$ has a scintillation efficiency of about $12 \%$. Thus, $1.2 \times 10^{5} \mathrm{eV}$ in total energy (or $4 \times 10^{4}$ photons) is produced by absorption of $1 \mathrm{MeV}$ energy. Each photon would have $3 \mathrm{eV}$ of energy on average [9].

$\mathrm{NaI}(\mathrm{Tl})$ can be produced in large crystal sizes and shapes by simple pressing techniques. It deteriorates if exposed to the atmosphere. Therefore, the crystal must be contained in air-tight casing for use. It is fragile and can be damaged easily by mechanical or thermal shock. Nevertheless, due to the large size with high light yield leading to good energy resolution, and shorter decay time, $\mathrm{NaI}(\mathrm{Tl})$ is the dominant scintillator in mobile spectrometry.

\subsection{Efficiency}

The efficiency of the detector system depends on many material and geometrical factors which in turn determine how well it provides a signal from the incident 
radiation. The efficiency is of both geometric and intrinsic types. Geometric efficiency is directly proportional to the radiation-sensitive detector volume and. for a point source, inversely proportional to the square of distance of the source to the detector. Intrinsic efficiency is the fraction of those radiations intersecting the detector that interact within the detector $[27]$.

\subsection{Our Instrument}

In this experiment, four RSX-1 [28] gamma detectors of $\mathrm{NaI}(\mathrm{Tl})$ were used with each crystal sized at $10.16 \times 10.16 \times 40.64 \mathrm{~cm}^{3}$. Each of the four $\mathrm{NaI}(\mathrm{Tl})$ crystal detectors has its own high speed $(60 \mathrm{Mz})$ Analog to Digital Converter (ADC) and processor assembly. The detector system also includes a console and laptop. In addition there is a GPS antenna, a 100-240 VAC power adapter, and cables, as shown in Fig. 8.

This system was developed by Radiation Solutions Inc. [28]. It works as a common multichannel analyzer in which each spectrum is given a geographical coordinate by the associated GPS.

The sampling time used depends on the sensitivity of the crystals used in the detector system. This system has long enough sampling time to get proper statistics but at the same time, the sampling time is short enough to give good spatial resolution. In practice, sampling times from $1 \mathrm{~s}$ to $10 \mathrm{~s}$ are commonly used. In this case, the sampling time is $1 \mathrm{~s}$.

The variation in any measured spectrum is huge as the gain changes while the system warms up. The system uses built in algorithms which stabilize the signal by adjusting the gain so that the natural radionuclide peaks always occur at the same channel in the spectrum. It does not require test sources for system stablization as this detector system uses natural radionuclides in the background.

The dead time is the minimum time separation between two signal pulses during 


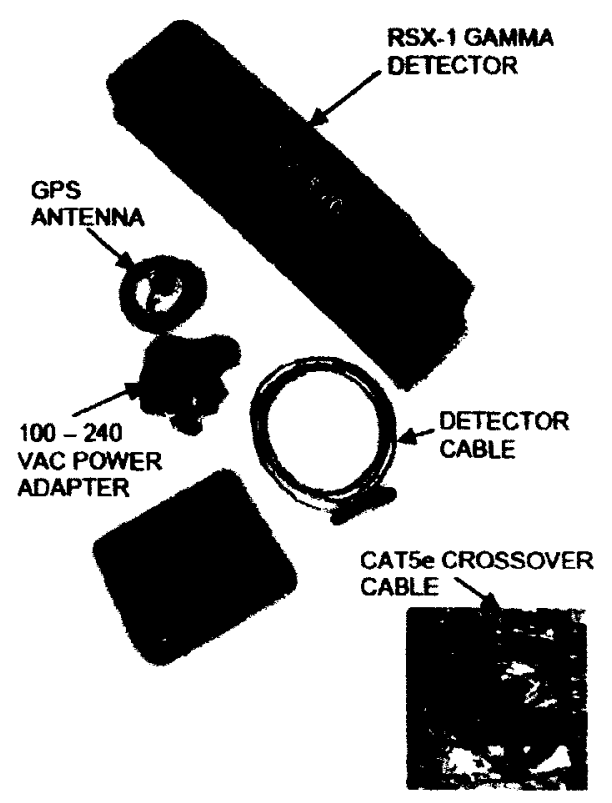

Figure 8: Different detector system's components are shown, namely GPS antenna, one of four detectors and cables [28](used under permissions).

which no information is recorded. Manufacturers report the dead time specific to their detector system. Thus, the dead time loss refers to the absence of signal during this period. This detector has, effectively, no dead time loss as fast pulse digitization enables pulse pile-up rejection and the only dead time comes from the intrinsic recovery time of the $\mathrm{NaI}(\mathrm{Tl})$ crystals. Detection rates for the source strengths and distances considered in this experiment were so low that the dead time was not a consideration.

There are 1024 channels in this detector system for fast data aquisition. The detector system can convert the analog signal from the detector to the digital spectrum with good energy resolution [28]. 


\section{Chapter 4}

\section{Methods}

There are different ways to perform source localization, depending on whether a detector is segmented or not. The first method used in this study involves off-line reconstruction of the source location using gross counts summed over all detector components. The second method involves extracting the direction to the source from count-rate ratios among the detector components. Background subtraction is also required as the most significant distortion in the data comes from the background due to naturally occurring radionuclides.

\subsection{Previous work}

\subsubsection{Source detection}

To distinguish natural from artificial radioactivity, one approach is to survey the ratio between the count rates at low spectral energies relative to those at higher energies [29]. Another approach is to find the ratio between the count-rate for different low-energy windows [30].

In one approach [7], fixed and moving background methods were devised for two point sources, Cs-137 and I-131. Data were obtained using $\mathrm{NaI}(\mathrm{Tl})$ detectors. In the 
methods adopted, the average gross counts in a spectral window from a test group were compared with pre-estimated fixed background. The $6 \mathrm{GBq}$ Cs- 137 source was detected at critical distances as far as $180 \mathrm{~m}$ and $4.5 \mathrm{GBq} \mathrm{I}-131$ at $170 \mathrm{~m}$. The critical distance is the maximum distance from the road possible in order for a source to be detected. The speeds were $32 \mathrm{~km} / \mathrm{h}$ and $54 \mathrm{~km} / \mathrm{h}$ with $3 \mathrm{~s}$ sampling time. The higher speed covers a larger area. This research was extended and compared [6] with three new moving-average algorithm-based methods involving different natural background subtraction (stripping) techniques to estimate critical distances for source location. These works cover the longer distances (up to $180 \mathrm{~m}$ ) and in the present thesis work, the shorter distances of $2 \mathrm{~m}, 12 \mathrm{~m}$ and up to $32 \mathrm{~m}$, are covered.

The method adopted by Creswell in 2009 [31] is based on a difference spectrum and is determined by the rolling average background and subtracting it from the measured spectrum. After the application of filters, the total count rate is calculated for the difference spectrum and compared with the statistical uncertainty of the measurement. This filtered background is then subtracted from the measurement and the process is repeated for all measurements. This technique is used in mapping and different case studies are included in the work.

In the present work, we looked for the characteristic photopeak of the source to stand out significantly above the natural background.

\subsubsection{Source localization}

A car-borne source localization method based on a $\mathrm{NaI}(\mathrm{Tl})$ detector was devised [2]. In this method, a Cs-137 source was placed at different distances from the road and the net count-rate profiles for each position were plotted. The speed of the car was $40 \mathrm{~km} / \mathrm{h}$ and sampling time $2 \mathrm{~s}$. The relationship between the full width at half maximum (FWHM) of the $662 \mathrm{keV}$ peak and the distance from the road was found based on those count rates. 
In the method above. the source was not shielded. A method devised by Hjerpe and Samuelsson in 2006 [32] used a semi-shielded Cs-137 source. A curve fitting technique was applied using the given regression model. The mathematical situation was described by Coll [33]. It turns out the curve fitting technique they used underestimates the distance, especially for longer distances, and the regression model needs to be improved upon.

Some work has been done where simulated data were suprerimposed on measured data to localize point sources [34]. These works are based on the gross count rates received by the detectors and most of the methods are applied during post processing of the data. These approaches, based on NaI(Tl) detectors, give source to road distance but lack directional information. One reason for this study is to test the directional method and so the gross count method was repeated under identical experimental circumstances to that of our directional measurements. Another reason is that the earlier studies were not performed for Na-22 and Cs-137 at the distances in the present work.

The literature also includes research on directional sensitivity [35] and the direction to the source by an angle using a portable directional radiation detector [36]. These studies offer only the direction to the source while directional source localization means finding a source position through triangulation using directional vectors. In the present work, directional source localization was performed.

In 2007, Uher et. al. [37] devised a method in which the count rate in a series of semiconductor detectors arranged in a column is measured and compared the count rate in each of the other detectors. This information is then used to determine the direction of the radiation. The simulated relative count profile was fit with a function that gives the count rate for $\mathrm{i}$-th detector. The angle of irradiation was determined within an accuracy of five degrees.

Different kinds of gamma imagers are under development for survey work, for 
example, pinhole or coded aperture imagers and Compton gamma imagers ( [38], [39]. [40]). A Compton imager is an electronically collimated radiation detector in which an incident gammma ray scatters in a primary detector while the interaction of scattered photon in a secondary detector is detected in coincidence and the cone, defined by Compton kinematics, gives the source location. Localization is achieved by finding the intersection of many Compton scatter cones [41].

The present study looks at a simple directional detector and not an imager. The imagers are not yet commercially available and are mostly not mature enough to take to the field. This study is to exploit the capabilities of an instrument which is commercial, off-the-shelf and field ready.

The gross count method is fairly mature while the directional methods are quite new and there is not yet a standard approach. Hence, there was a need to develop a method that would provide us with directional ability as well. Therefore, two methods were developed, called the gross count method and the directional method.

\subsection{Experimental setup}

A non-residential location was chosen to perform the experiment for source localization. The detector system was mounted carefully inside a truck. Radioactive sources (Na-22 and Cs-137) were placed at positions A, B, C, D, E, F (2 m, $12 \mathrm{~m}$, $22 \mathrm{~m}, 32 \mathrm{~m}, 42 \mathrm{~m}$ and $52 \mathrm{~m}$, respectively), as indicated on Fig. 9a, with each position separated by $10 \mathrm{~m}$ distance. Each measurement was taken with one source at one position at a time. That is to say, the source was first placed at $2 \mathrm{~m}$ (position A) distance and measurements were taken. Once the measurements were completed, the source was shifted to the next position. and so on. The strength of the Na-22 source used was $\sim 0.037 \mathrm{GBq}(1 \mathrm{mCi})$. The strength of $\mathrm{Cs}-137$ was less than $\sim 1 \mathrm{mCi}$. 
a)

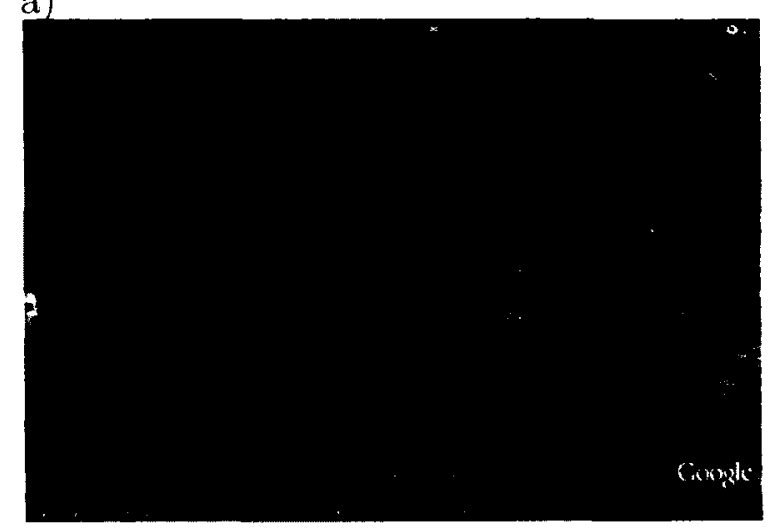

b)

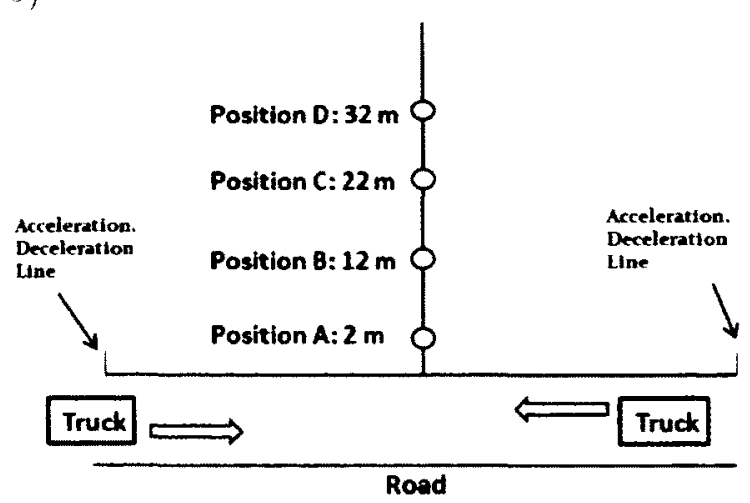

Figure 9: a) The Google map of the place where experiment was conducted. The pink lines are the tracks of the truck and A, B, C, D, E and F give the positions of gamma emitting sources [42] (used under Google's attribution and fair use policy). b) Schematics of the setup with sources at positions up to D.

Fig. 9 shows the location of the experiment with the track of the truck indicated by pink lines. The acceleration and deceleration lines were marked on the track. At the acceleration line, the truck was made to run at the required velocity and this velocity was maintained throughout the duration of the pass till the decelaration line was reached. The truck was made to pass by these sources at different velocities, i.e., $20 \mathrm{~km} / \mathrm{h}$ and $40 \mathrm{~km} / \mathrm{h}$, sixteen times. The data were collected on August 24 and 25 , 2011. Background runs ( 8 passes) were also performed when the sources were not present.

\subsection{Gross Count method (GC)}

When it is necessary to identify or find a source location by analysing the data from $\mathrm{NaI}(\mathrm{Tl})$-based measurements, the most common method is to evaluate the gross counts in the detectors (in the present case, the four detectors) used. Here, gross counts refer to summing the photopeak counts of all of the detectors, as opposed to looking at each detector separately. 


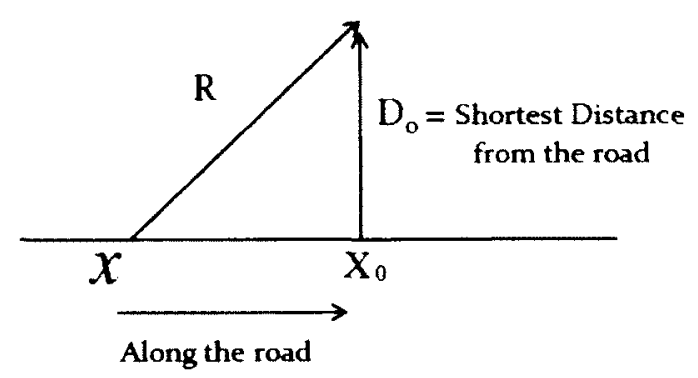

Figure 10: A truck moves by the source placed at a perpendicular distance $D_{0}$ from the road.

Fig. 10 shows a schematic of the gross count method, where $D_{0}$ is the shortest distance of the source from the road, $x$ gives the position of the truck as it moves on the road, $\mathrm{x}_{0}$ is the truck's position where the distance of the source from the road is shortest and $R$ is the distance from the truck to the source position. As the truck moves closer to the source, this distance will vary as well. We are interested in determining the distance $D_{0}$ from the measured background-subtracted photopeak counts received by the $\mathrm{NaI}(\mathrm{Tl})$ detector as a function of the position along the road, $x$.

The count rate follows the inverse square law, that is, it is inversely proportional to the square of the distance of the source from the detector, taking into consideration the air attenuation. The expected count rate as a function of $R$ is thus,

$$
C(R)=\frac{1}{4 \pi R^{2}} N A e^{-\mu_{a i r} R}
$$

where ,

$$
R^{2}=\left(x-\mathrm{x}_{0}\right)^{2}+D_{0}^{2}
$$

In Eq. $9, N=S \epsilon B$, where $S$ is the source strength (in $\mathrm{Bq}$ ), $\epsilon$ is the detector's efficiency and $B$ is the branching ratio for the radionuclide used. Also in Eq. $9, A$ is 
effective area of the detector and $\mu_{\text {air }}$ is the linear attenuation coefficient of air $\left(\mathrm{m}^{-1}\right)$.

Fig. 11 shows the dependence of the count rate on distance of the source from the road for four different positions calculated using Eq. 9 which predicts a wider profile if the source is farther away. From Fig. 11, it is clear that a source farther from the road (say at $30 \mathrm{~m}$ ) gives a broader profile, while a near source at $5 \mathrm{~m}$ gives a narrower profile, as is evident from the width of the curves.

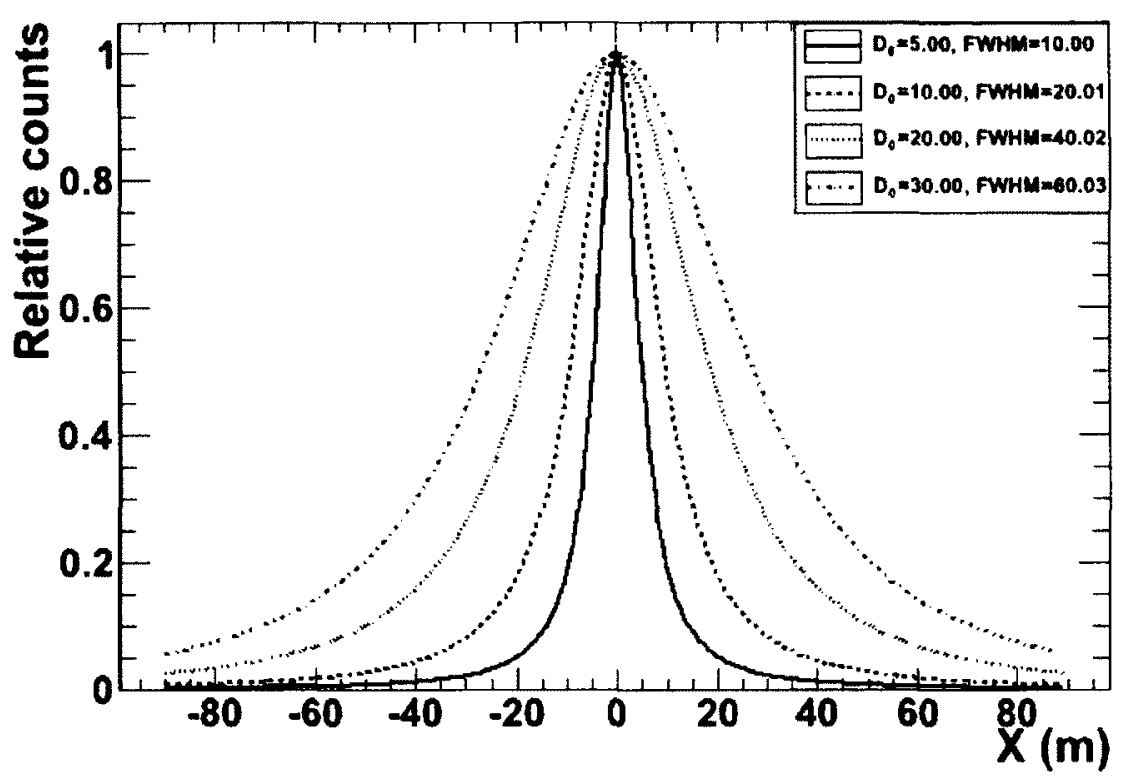

Figure 11: The theoretical dependence of the count-rate on distance along the road (X) for four different values of $D_{0}$, the distance from the road.

In the gross count method, Eq. 9 was fit to the total counts received by all detectors to find the position along the road $\left(x_{0}\right)$ and the position of the source from the road $\left(D_{0}\right)$. Based on these calculations, the reconstructed source positions were determined. 


\subsection{Directional Method (DM)}

In the directional method, the direction to the source was determined by forming a directional vector based on the photopeak counts in each of the four detectors. Fig. 12a shows four $\mathrm{NaI}(\mathrm{Tl})$ detectors (arranged vertically) with letters $C_{i}$ representing photopeak counts from each detector. The directional vector $\vec{V}(\mathrm{u}, \mathrm{v})$, based on the counts received by the detectors, is formed by the components $u$ and $v$ given by,

$$
\begin{aligned}
& \mathbf{v}=C_{0}-C_{2}, \\
& \mathbf{u}=C_{1}-C_{3} .
\end{aligned}
$$

The directional vector, $\vec{V}$ is given by,

$$
\vec{V}=(\mathrm{u}, \mathrm{v})
$$

where $u$ and $v$ are scalar quantities. These are the lengths of the vector $\vec{V}$ projected along the basis vectors $\hat{\mathrm{u}}$ and $\hat{\mathrm{v}}$.

Fig. $12 \mathrm{~b}$, shows the directional vector $\vec{V}$ formed by the components $v$ and $u$, which in turn are determined by the difference in the photopeak counts given by Eq. 11 and Eq. 12 , respectively. The angle between the vector $\vec{V}$ and the basis vector $\hat{u}$, is given by,

$$
\theta=\operatorname{atan} 2(\mathrm{u}, \mathrm{v})
$$

The double length inverse tangent (or arctangent), represented by math routine atan2 (a specific implementation of more general formula atan), is used in this case, to cover all the angles in all four quadrants, that is, even for the negative values. The 
a)

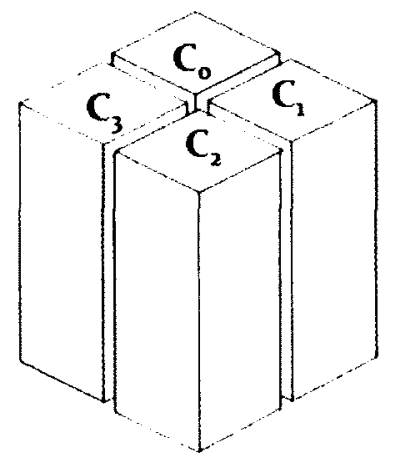

b)
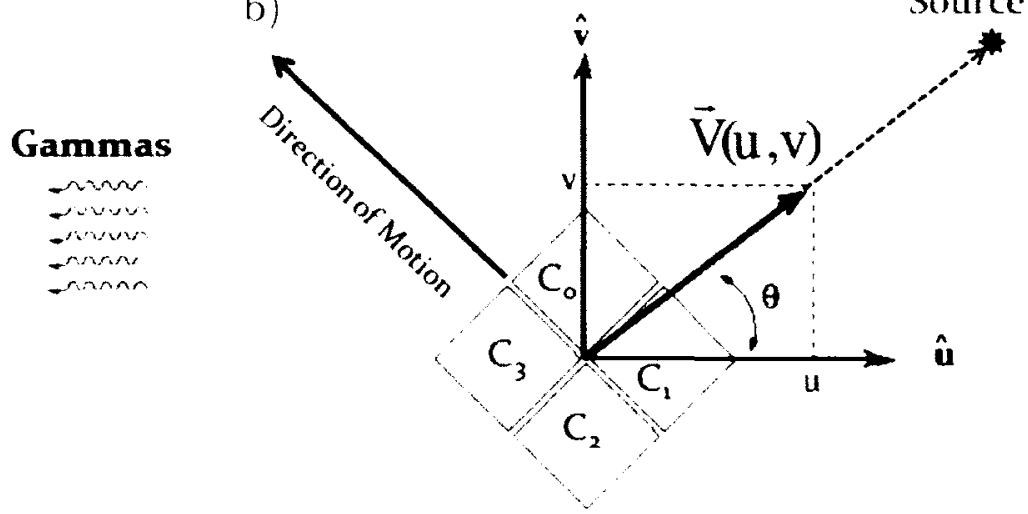

Source

Figure 12: a) The detectors' orientation with assigned letters representing respective counts in each detector, and b) the directional vector pointing towards the source along with the direction of motion of the detectors.

range of $\operatorname{atan} 2$ is $[-\pi, \pi]$.

The angle between $\vec{V}$ and the vector defining the forward motion of the truck is given by,

$$
\theta^{\prime}=\frac{3 \pi}{4}-\theta
$$

The $\frac{3 \pi}{4}$ accounts for the fact that the orientation is such that the detector is rotated by some multiple of $45^{0}\left(\frac{\pi}{4}\right)$ from the forward orientation of the truck.

The detectors are self-shielded, which means that only the detectors in front, facing the source, would detect the gamma counts and would shield the detectors behind them.

The angle between the direction of the truck's movement and the East (E) direction, is given by,

$$
\Phi=\operatorname{atan} 2\left(x_{i}-x_{(i-1)}, y_{i}-y_{(i-1)}\right)
$$

where $x_{i}$ and $y_{i}$ are the Easting and Northing coordinate pair giving the truck's position at any given time $i$ and $x_{(i-1)}$ and $y_{(i-1)}$ give the truck's position at the 
previous time $(i-1)$.

$\theta^{\prime}$ and $\Phi$ were then used to find the angle $\Psi$; the angle between the vector $\left.\vec{V}(u, v)\right)$ and the East (E) direction, as shown in Fig. 13.

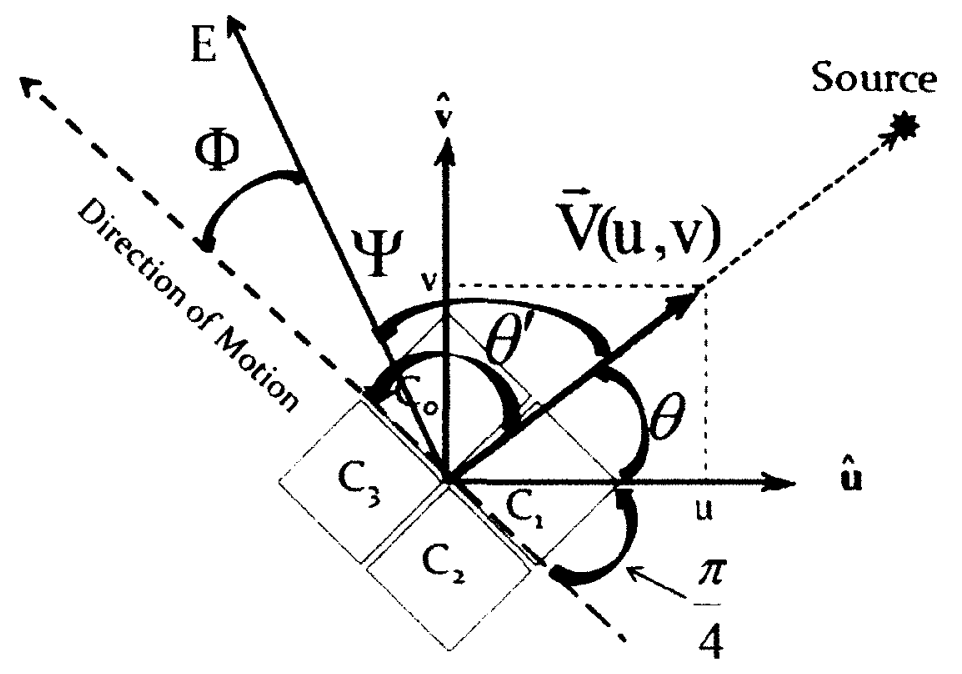

Figure 13: The angle $\Psi$ between the vector $\vec{V}(\mathrm{u}, \mathrm{v}))$ and the East direction.

The desired angle $(\Psi)$, is given by,

$$
\Psi=\theta^{\prime}-\Phi
$$

Based on position coordinates, the movement of the truck was tracked, back and forth on the road as it was detecting the gamma counts. These angle-equations helped in giving direction to the probable source location in the form of arrows (directional vectors).

\subsubsection{Derivation of directional vectors for limiting cases}

The gamma rays are attenuated as per the attenuation law given by Eq. 6. Two limiting cases for the derivation of the directional vector are discussed, assuming that the detectors are extremely thick and material is extremely dense, that is, $\lambda \Delta L$ is 
very large.

Case 1: Source on side of the four detectors array at $\theta=45^{\circ}$

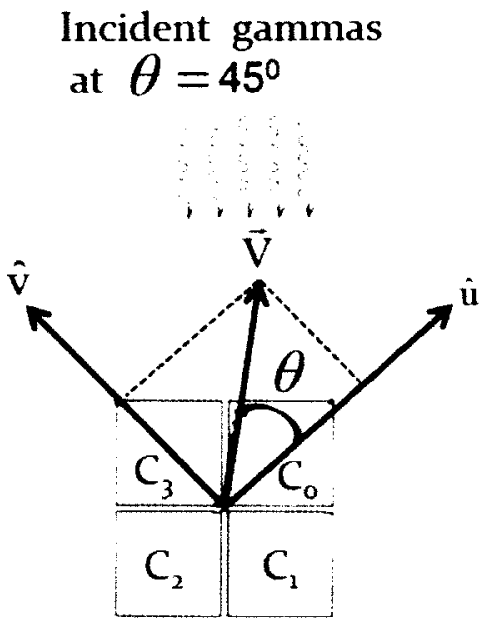

Figure 14: The orientation of two of the detectors facing radiation source at $\theta=45^{\circ}$ and the directional vector $\vec{V}$.

The source is at the side of the array of the four detectors at $45^{\circ}$ angle, as shown in Fig. 14. In the limiting case, $\lambda \Delta L \rightarrow \infty ; C_{3}=C_{2}=0$. The counts received in detectors facing the source are the same and as the detectors are self-shielded, the counts received at the detectors on the opposite side are zero. The components $\mathrm{v}$ and $\mathrm{u}$ are given by,

$$
\begin{aligned}
& \mathrm{v}=C_{0}-C_{2}=C, \\
& \mathrm{u}=C_{1}-C_{3}=C .
\end{aligned}
$$

The directional vector based on counts, in this case, is given by,

$$
\overrightarrow{\mathrm{V}}=(C, C)
$$

The reconstructed angle $\theta$ is thus $45^{\circ}$, as expected. 
Case 2: Source at $\theta=0^{0}$ to the four detectors array

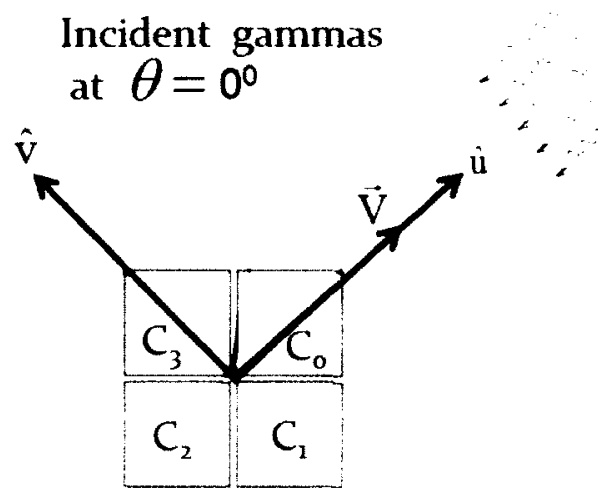

Figure 15: The orientation of the detectors with the radiation source at $\theta=0^{0}$ and the directional vector $\vec{V}$.

If the source is at $0^{0}$ to the four detectors array then, from Fig. 15, it is clear that the counts would be received by all but one of the detectors, that is, $C_{3}=0, C_{1}=C$ and $C_{0}=C_{2}=C^{\prime}$.

The directional vector, based on counts, in this case, is given by,

$$
\overrightarrow{\mathrm{V}}=(0, C)
$$

The reconstructed angle $\theta$, in this case, is thus $0^{\circ}$, as expected.

\subsubsection{Directional calibration}

A directional calibration experiment was performed at the National Research Council (NRC). The detector system was transferred and set up in one of the labs at NRC. On top of the detector, true angles $\left(0^{0}\right.$ to $90^{\circ}$ and $0^{0}$ to $\left.-90^{\circ}\right)$ were marked. After setting up the detector system, directional data were taken with a $1 \mathrm{mCi}$ Cs-137 source placed at an angle $\left(\theta_{\text {true }}\right)$. The process was repeated for true angles in $10^{\circ}$ intervals from $-90^{\circ}$ to $90^{\circ}$. The measured angles $(\theta)$, were then plotted against the 
true angles $\left(\theta_{\text {true }}\right)$, as shown in Fig. 16.

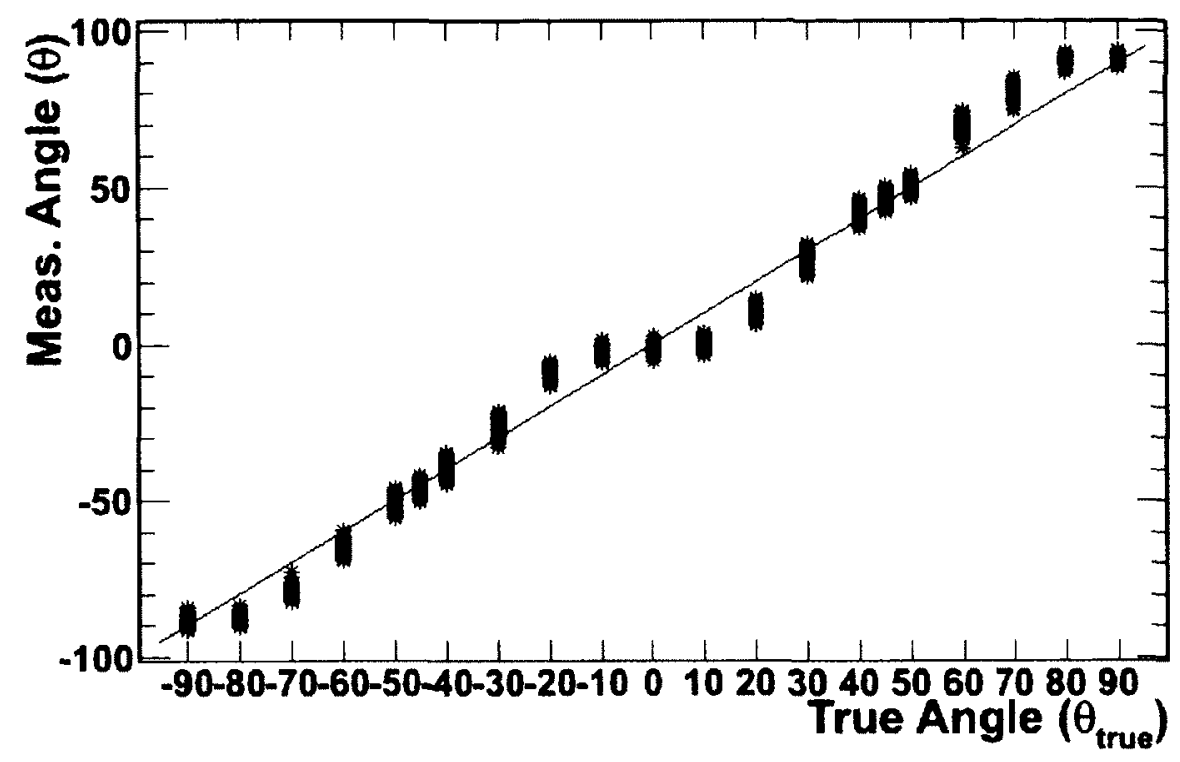

Figure 16: Directional calibration: the correlation between the true angle and the measured angle in degrees.

From Fig. 16, it can be seen that there is a positive correlation between measured angles $(\theta)$ and true angles $\left(\theta_{\text {true }}\right)$, so it is clear that the method to reconstruct directional vector $\vec{V}(\mathrm{v}, \mathrm{u})$ from the count differences is valid. There is, however, some deviation from the 45 degree line, which will be discussed further in the following.

The angular resolution plots for angles, $-45^{\circ},-10^{0}, 10^{\circ}$ and $45^{\circ}$ are shown in Fig. 17. 
a)

c)
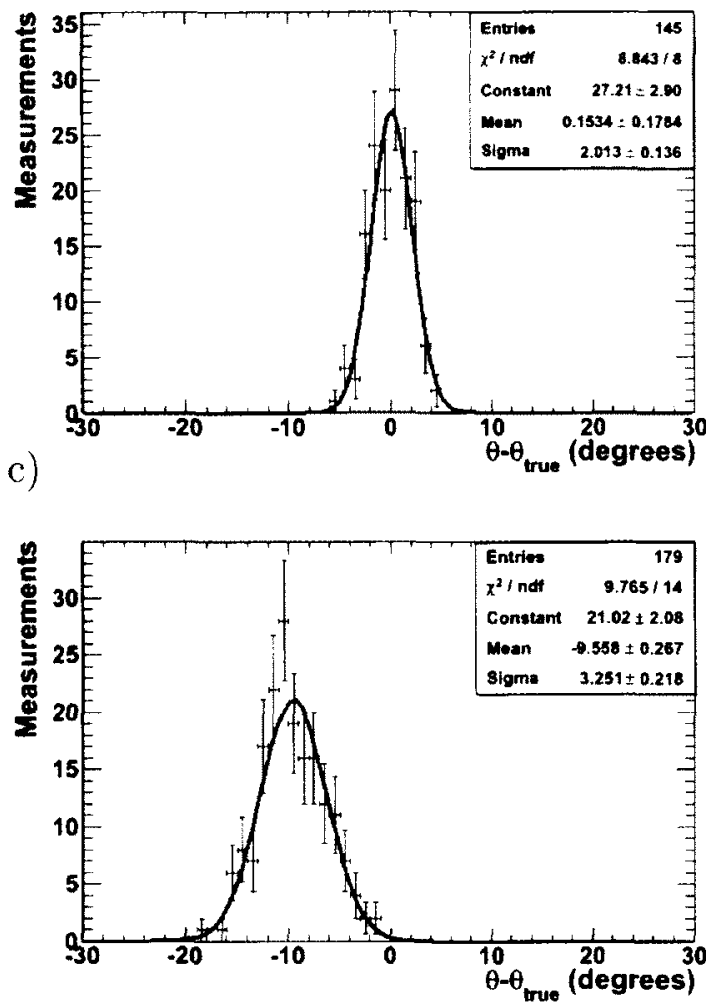

b)
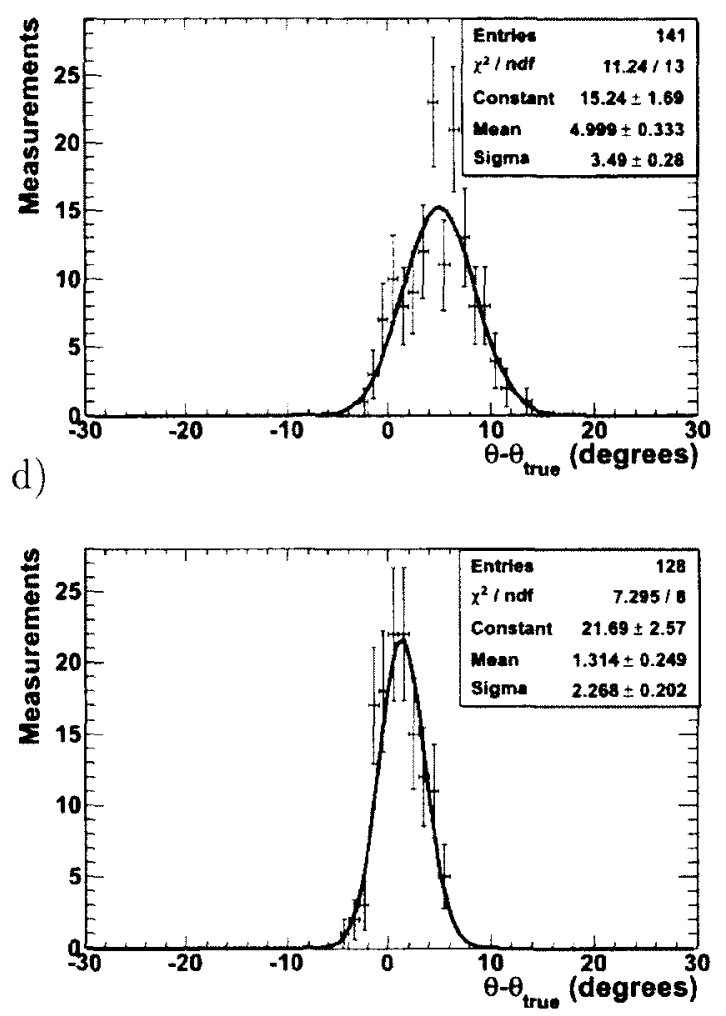

Figure 17: The distribution of the difference between the measured angle and true angle $\left(\theta-\theta_{\text {true }}\right)$, for 2-3 minutes-worth of one-second measurements of $\theta$ at a) $-45^{\circ}$, b) $-10^{0}$, c) $10^{\circ}$ and d) $45^{\circ}$. 
These angle-difference plots $\left(\theta-\theta_{\text {true }}\right)$ for angular resolution are centered near zero for $-45^{\circ}$ and $45^{\circ}$, as would be expected from symmetry. But there is a systematic offset for the in-between angles (say $-10^{0}$ and $\left.10^{\circ}\right)$, as shown in Fig. 17. The angular resolution, as taken from the width of the individual Gaussian fits, is two to three degrees, neglecting the systematic offset affecting some angles.

The experiment was difficult to set up due to the presence of different equipment and space. The true angle measurements were taken manually using a laser-pointer. The small offsets which are seen in the $-45^{\circ}$ and $45^{\circ}$ measurements, are likely due to the mis-measurement of the true angle $\left(\theta_{\text {true }}\right)$ rather than a real offset in the determination of measured angle $(\theta)$.

The angular resolution averaged over all true angles is shown in Fig. 18.

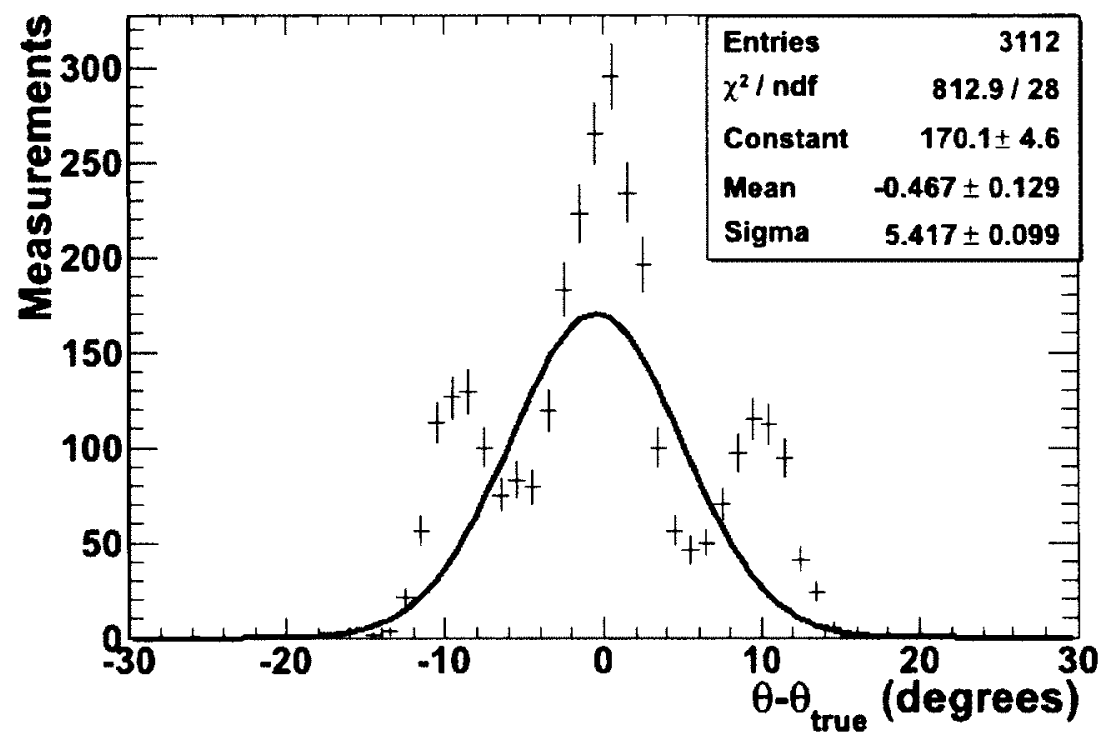

Figure 18: The angular resolution averaged over all true angles when the offset is not corrected for.

A Gaussian fit to this distribution is bad because the data clearly exhibit large tails outside of a central distribution. Believing the Gaussian fit, the angular resolution would be $\sim 5.4^{0}$. 
The means of the angle-difference $\left(\theta-\theta_{\text {true }}\right)$ for all angles were then plotted against the measured angles $(\theta)$, and fitted with the sum of two sine functions each with independent offset and amplitude, as shown in Fig. 19.

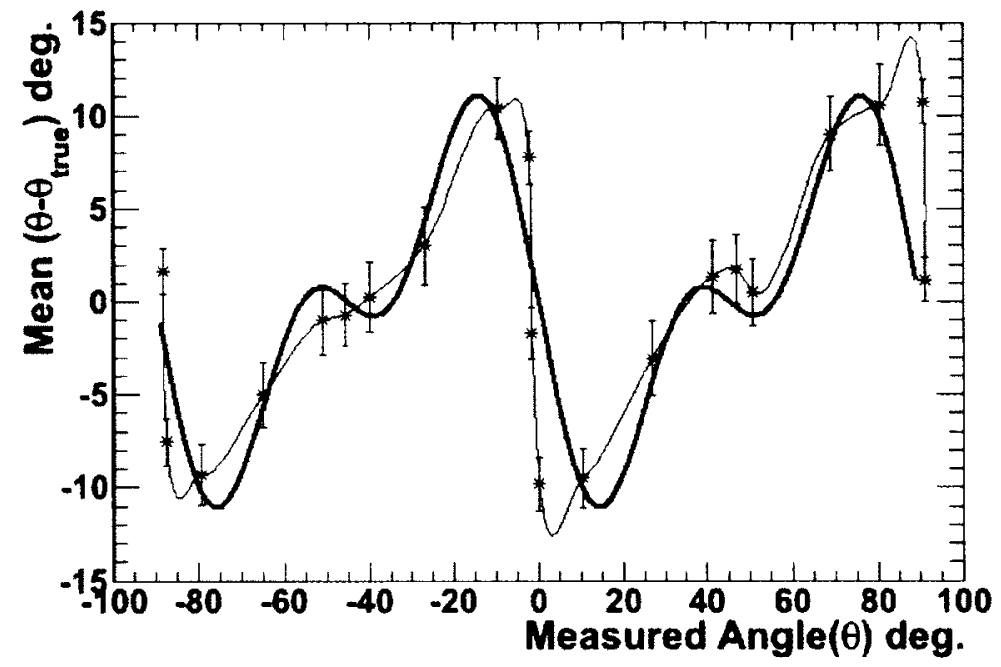

Figure 19: Means of the angle difference $\left(\theta-\theta_{\text {true }}\right)$ against the measured angle $(\theta)$. The stars show the data, with the error bar representing the width of the distribution of measured angles. The curves show the result of a fit to the sum of two sine functions.

From Fig. 19, it can be seen that there is a large offset of up to $10^{\circ}$ for the intermediate angles between $0^{\circ}$ and $45^{\circ}$. The offset appears to be low at $-90^{\circ},-45^{\circ}$, $45^{\circ}$ and $90^{\circ}$.

The fit shown in Fig. 19 represents the function $F$ which can be used to correct the measured $\theta$ values,

$$
\mathrm{F}=(-7.5) \sin 4 \theta+(-5.2) \sin 8 \theta
$$

Correcting for the offset using Eq. 22, the angular resolution, averaged over all true angles was determined again, as shown in Fig. 20. By correcting for the offset, the angular resolution was improved from $\sim 5.42^{0}$ to $\sim 3^{0}$. The tails outside of the central Gaussian distribution are much smaller after the correction for the reconstructed angle off-sets. 


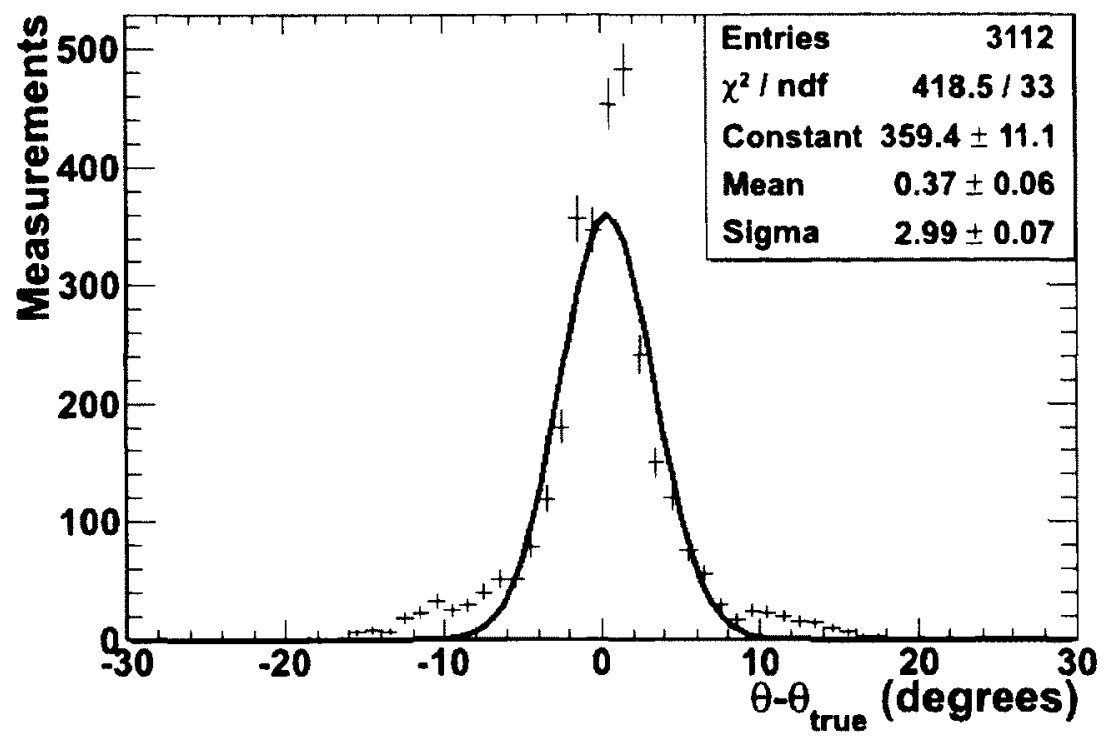

Figure 20: The angular resolution averaged over all angles when the offset is corrected for.

In the following, the correction for the offset has been applied to the reconstruction of the source position in the directional method.

\subsubsection{Probable source location}

The directionality in this method is represented by arrows based on counts, pointing towards the source location as the truck passed by the source. The closest approach to the source gave maximum counts. The length of the arrow is,

$$
\vec{V}=\sqrt{u^{2}+v^{2}}
$$

and the position of the origin of the arrow is the position of the truck (corrected for lag, to be discussed in Chapter 5, section 5.3). Fig. 21 shows an example of the arrows reconstructed from one pass past the Na-22 source, as a function of the position of the truck. Far from the source, natural background dominates, and the 


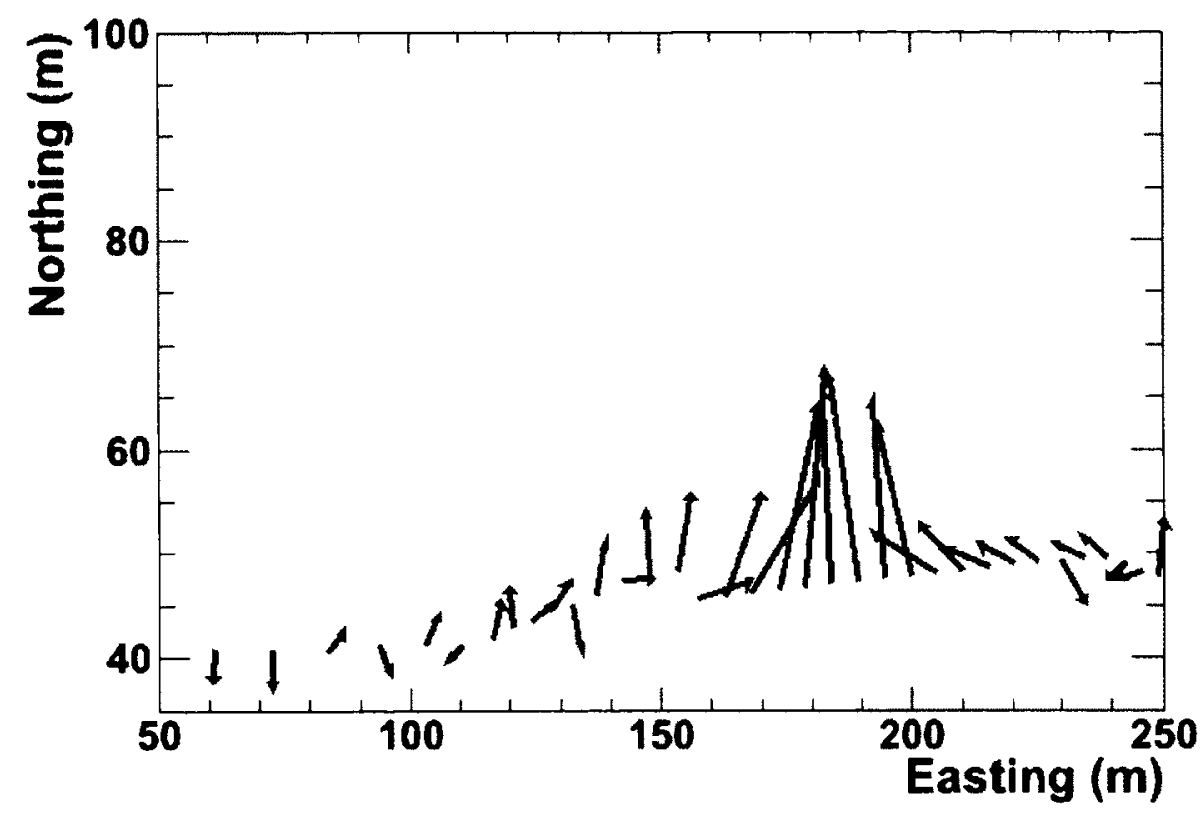

Figure 21: Northing versus Easting is shown, for $\mathrm{Na}-22$ at $20 \mathrm{~km} / \mathrm{h}$ at position C. The direction arrows $(\vec{V})$ are plotted with origin at the truck's (corrected) position, with length of the arrows given by Eq. 23 .

arrow direction is random. Near the closest approach to the source, the arrows point to a common probable source location.

A point $\mathrm{P}$ is selected in the probable source region. From that point, the intersecting lines of lengths $d_{1}, d_{2}, d_{3}, \ldots d_{N_{a r r o w s}}$, were drawn to all of the arrows as illustrated in Fig. 22. The distances $d_{i}$ are found from the point $\mathrm{P}\left(x_{1}, y_{1}\right)$ to the arrow, which has origin $\left(x_{2}, y_{2}\right)$, according to,

$$
d_{i}=\left|\sqrt{\left(x_{1}-x_{2}\right)^{2}+\left(y_{1}-y_{2}\right)^{2}} \times \sin (\alpha)\right|
$$

where,

$$
\alpha=\operatorname{atan} 2\left(y_{1}-y_{2}, x_{1}-x_{2}\right)-\beta
$$




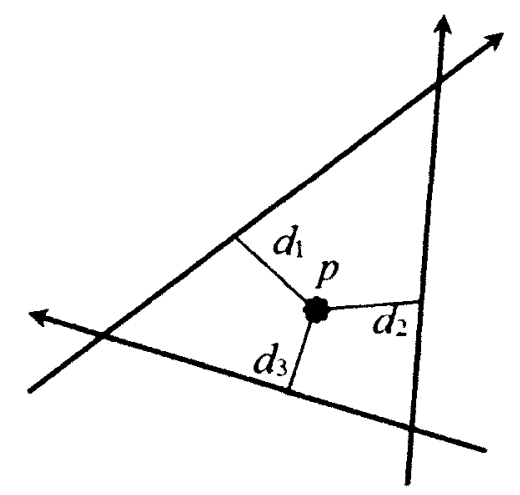

Figure 22: A point $\mathrm{P}$ was selected in the probable region of the source and lines were drawn to all the arrows.

and where,

$$
\beta=\text { Angle of arrow with respect to East }=\theta^{\prime}-\Phi .
$$

After calculating all the distances from the point $\mathrm{P}(x, y)$ to the arrows, a distance function was developed. This distance function is the sum of the squares of all the distances, given as,

$$
D(x, y)=\sum_{i=1}^{N_{\text {arrows }}} d_{i}^{2}(x, y),
$$

where $x=\operatorname{Easting}(\mathrm{m})$ and $y=\operatorname{Northing}(\mathrm{m})$ and $d_{i}$ is the shortest distance between $i^{\text {th }}$ measured vector and the point $\mathrm{P}$. The resulting distance function is a surface plot, as shown in Fig. 23.

This distance function was minimized using Minuit [5] to localize the source. In other words, a minimization algorithm was used to find the minimum of $\mathrm{D}(\mathrm{x}, \mathrm{y})$, to reconstruct the source position. 


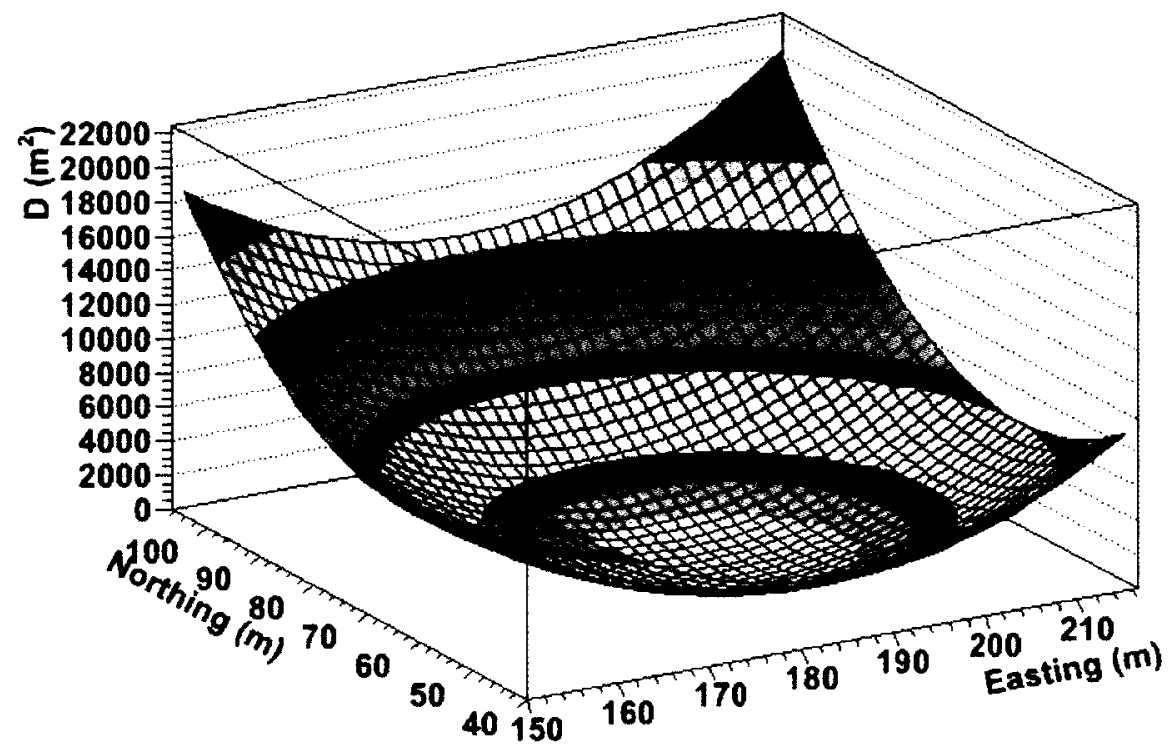

Figure 23: The distance function as a function of position shown in the form of a surface plot. 


\section{Chapter 5}

\section{Results and Analysis}

\subsection{Energy spectra for source identification}

\subsubsection{Background energy spectrum}

The distortion in post processed data for gross counts, comes mainly from the naturally occuring radionuclides. Therefore, before starting the experiment, a survey was performed with the same detector system for the natural background counts in the absence of the gamma source.

Artificial gamma sources typically emit photons with energies well below the energies of the characteristic photons from natural radionuclides, such as $1461 \mathrm{keV}$ from K-40, $1765 \mathrm{keV}$ for Bi-214 (from the uranium decay series) and $2615 \mathrm{keV}$ for Tl-208 (from the thorium decay series). The gamma photons get scattered by soil and that is one of the main reason for them to end up in lower energies where the range of energies of interest lies. Therefore, the energies of interest overlap with the energies of gammas down-scattered from the natural peaks.

The energies of interest fall in the energy windows $450-550 \mathrm{keV}$ and $600-710 \mathrm{keV}$, for Na-22 and Cs-137, respectively. Post processing analysis of the data was performed to get the total counts received in the absence of the source for the natural background 
in respective energy windows. A spectrum with the energy windows. as an example. and the averaged background counts in one of the energy window $(450-550 \mathrm{keV})$. thus measured, are shown in Fig. 24a and Fig. 24b. respectively.

a)

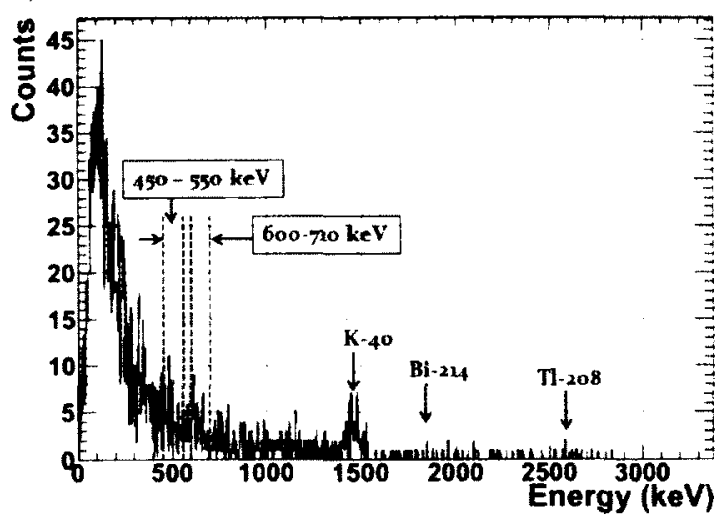

b)

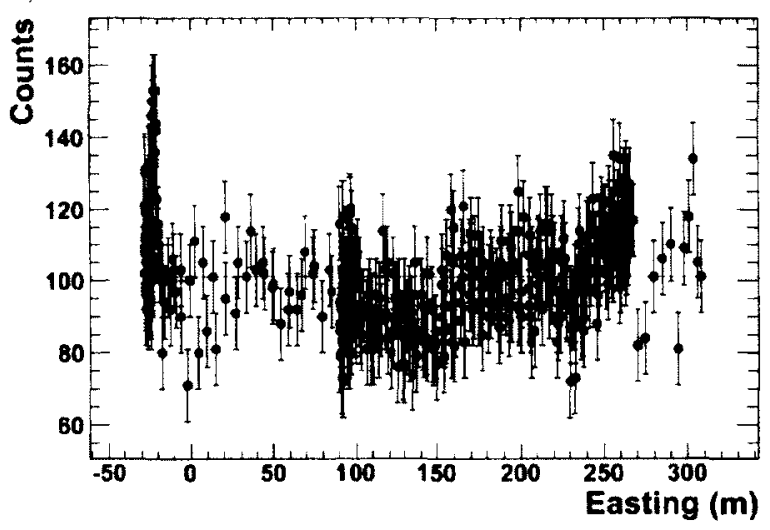

Figure 24: a) The energy spectrum of the background for one second. The energy windows of $450-550 \mathrm{keV}$ and $600-710 \mathrm{keV}$ are represented by vertical lines for Na-22 and Cs-137 respectively. Natural peaks are indicated by arrows. b) The measured average total counts for natural background in the energy window $450-550 \mathrm{keV}$ as a function of Easting along the truck's path. The solid line shows the mean of the counts.

The average of the background counts in the energy windows $450-550 \mathrm{keV}$ for Na-22 and 600-710 keV for Cs-137, were used in our calculations. The average of these total counts was then subtracted from the measured spectra which we obtained during post processing of the data in the gross count method.

Note that the directional method, which is based on count differences in the different detector components, should be relatively insensitive to the background. Therefore no background treatment was applied in the directional analysis.

\subsubsection{Na-22 energy spectrum}

An energy spectrum for Na-22, at position $\mathrm{C}$, is shown in Fig. 25. The spectrum is shown for a one second measurement. which is the sampling time, at the speed 


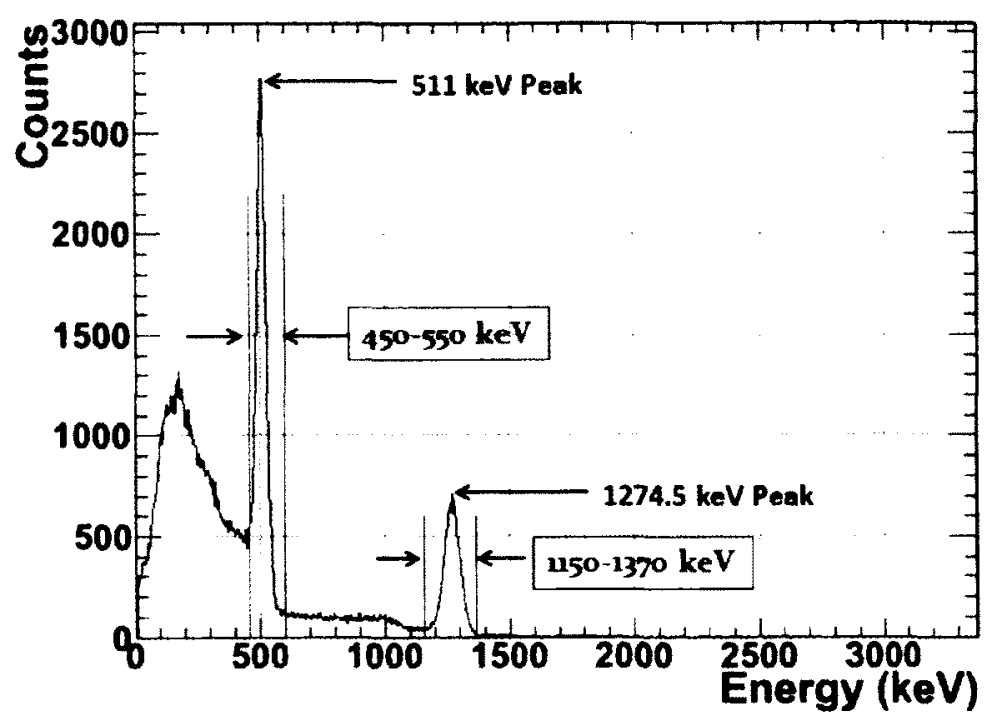

Figure 25: The energy spectrum for $\mathrm{Na}-22$, for one second with the source at position $\mathrm{C}$ and detector inside the truck on the road somewhere near the position of highest count rate. Two peaks at $511 \mathrm{keV}$ and $1274.5 \mathrm{keV}$ are the energies of the gammas given off by Na-22 in the energy windows of $450-550 \mathrm{keV}$ and $1150-1370 \mathrm{keV}$, respectively.

of $40 \mathrm{~km} / \mathrm{h}$, while passing by the source. The energy spectra were plotted and analysed to confirm the presence of the source, Na-22. The photopeak counts from the selected energy windows (450-550 keV) for $511 \mathrm{keV}$ gamma and another energy window (1150-1350 keV) were used to determine the type of source. The photopeaks corresponding to $511 \mathrm{keV}$ and $1274.5 \mathrm{keV}$ tell us the presence of $\mathrm{Na}-22$. It is a typical $\mathrm{Na}-22$ signature. The $511 \mathrm{keV}$ photopeak is due to a gamma photon that is produced as a result of positron-electron annihilation. The second photopeak at $1274.5 \mathrm{keV}$ is produced when a gamma comes off as a result of de-excitation of the daughter which decays to the ground state. The events that fall below the $511 \mathrm{keV}$ photopeak in the spectrum are due to the gammas which Compton scatter inside the detector and then escape. 


\subsubsection{Cs-137 energy spectrum}

Fig. 26 shows an energy spectrum for Cs-137. taken at position B while the detector was $12 \mathrm{~m}$ away inside the truck on the road. The energy window $(600-710 \mathrm{keV})$ is indicated. This spectrum is a one second measurement, taken at the speed of $40 \mathrm{~km} / \mathrm{h}$. The photopeak corresponding to the gamma energy of $662 \mathrm{keV}$ can be seen in Fig. 26. The events to the left of the photopeak correspond to escaping gammas. This spectrum represents a typical Cs-137 profile thus confirming the presence of Cs-137.

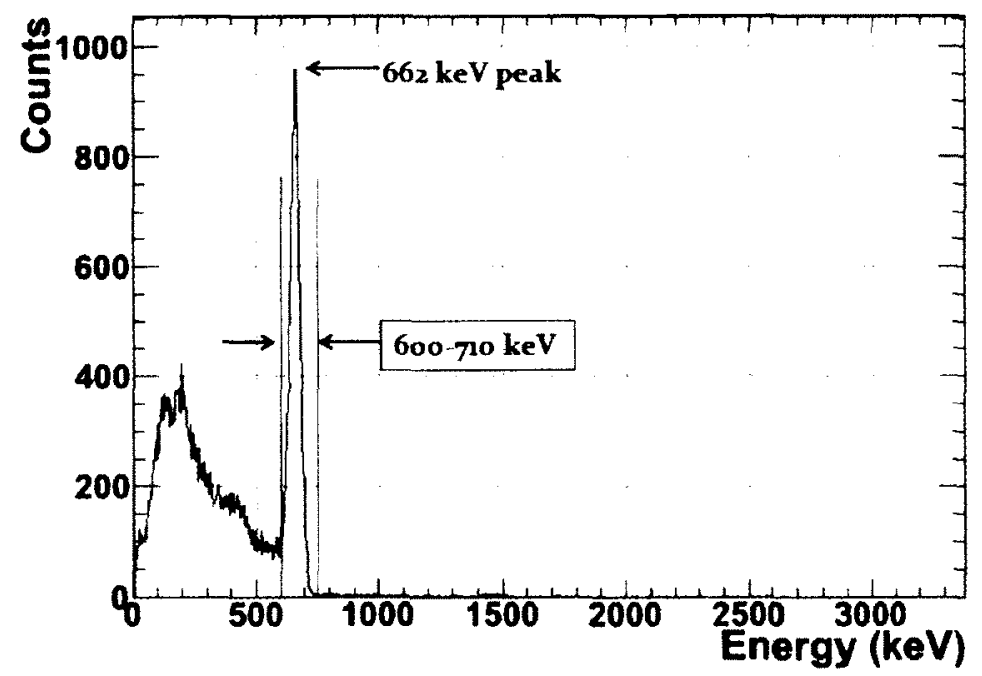

Figure 26: The energy spectrum of Cs-137, at position C for one second. The energy window $600-710 \mathrm{keV}$ is indicated. The photopeak is at $662 \mathrm{keV}$.

The energy spectra above have confirmed the presence of the Na-22 and Cs-137 sources and the subsequent step in the present work was to localize them.

\subsection{DGPS correction}

The instrument acquired data with a GPS antenna. Most GPS instruments give $7.8 \mathrm{~m}-15 \mathrm{~m}$ accuracy of horizontal positions. $95 \%$ of the time, on earth [43]. Modern 
ones, however. may give better accuracy with a clear sky view. But still, it is not considered very precise in many cases, for example, in scientific evaluations where localization of point sources is needed.

There are many sources of error in GPS acquired data. One source of error is the refraction in the atmosphere which alters the speed of the GPS signal. Another source of error is the distortion caused by the inherent electrical interference in the GPS itself. The error in the measured satellite's position would also cause error in computed positions.

The correction for the atmospheric error is performed by a method called differential correction employed in the Differential Global Positioning System (DGPS). The DGPS adds a local reference station to augment the information from satellites. This reference station transmits correction data in real time to the receiver included in the DGPS system.

In order to get better information on the coordinates of the truck's position during the experiment, the DGPS was used as well and these coordinates were applied during post processing of the data.

We occasionally had problems with the DGPS antenna power. So some of the data have the differential correction, but some does not. A DPGS can acheive an accuracy of $1 \mathrm{~m}$ to $5 \mathrm{~m}$. It improved the accuracy of the receiver used as can be seen from Fig. 27. There is still some residual left with the measurement of position which can be due to a non-optimal location of the satellites at the time of the survey. 

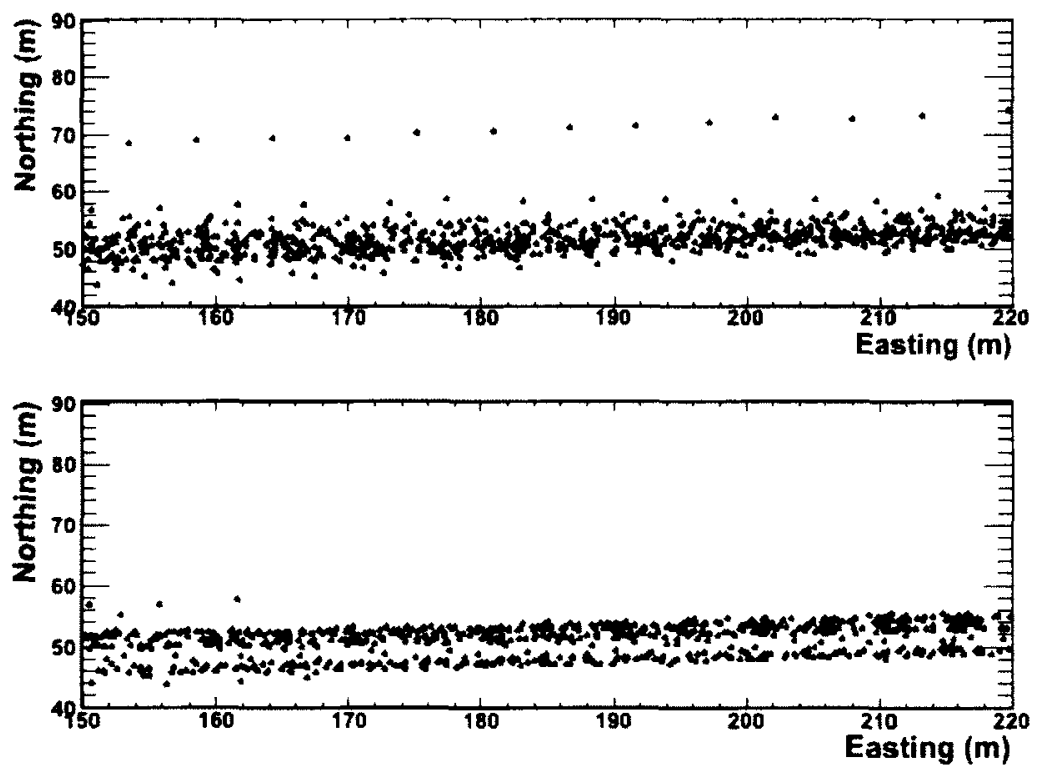

Figure 27: The DGPS error correction applied to improve the accuracy of the data. The data for which we had DGPS information are shown. The top figure shows the position data before the DGPS correction was applied and the bottom plot shows the position data after the DGPS correction was applied. 


\subsection{Lag correction}

In this experiment, the truck moves back and forth in an east-west direction with the detectors mounted inside. The detectors receive gamma counts as a function of position. Typical spectra are represented in Fig. 28 as a function of position.

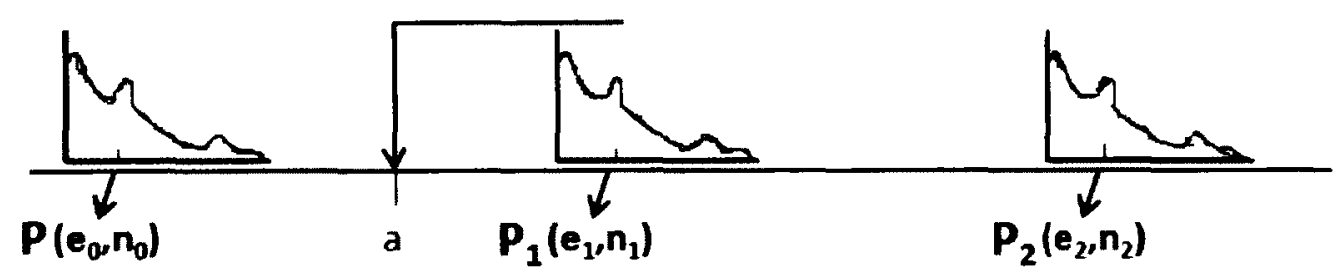

Figure 28: Schematics of typical spectra demonstrating the lag effect. The measured spectrum should actually be attributed to position 'a' marked in the figure.

The counts recieved at a position say, $\mathrm{P}_{1}\left(e_{1}, n_{1}\right)$ are lagged. They have been tagged with the geographic position $\mathrm{P}_{1}\left(e_{1}, n_{1}\right)$ corresponding to the truck's position at time $\mathbf{t}=\mathrm{t}_{1}$. But actually they come from every position between $\mathrm{P}\left(e_{0}, n_{0}\right)$ at time $\mathbf{t}=\mathrm{t}_{0}$ and $\mathrm{P}_{1}\left(e_{1}, n_{1}\right)$ at time $\mathbf{t}=\mathrm{t}_{1}$. Here, $e_{1}$ and $n_{1}$ represent the Easting and Northing of the point $P_{1}$. This lag effect is corrected using,

$$
\mathrm{l}_{e}=e_{0}+\frac{e_{1}-e_{0}}{2} ; \mathrm{l}_{n}=n_{0}+\frac{n_{1}-n_{0}}{2},
$$

where $l_{e}$ and $l_{n}$ give the Easting and Northing, after the lag correction has been applied, respectively.

Fig 29a and Fig. 29c, show the counts profile for the Na-22 source for two consecutive passes, one in the eastward direction and one in the westward direction, at two different positions ( $\mathrm{A}$ and $\mathrm{C}$ ), without the lag correction. One can see that the peak in counts is shifted in position by the lag effect. After the lag correction 
a)

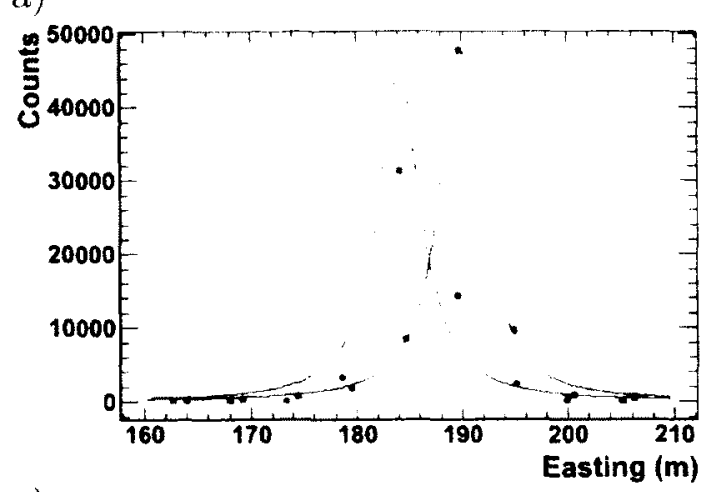

c)

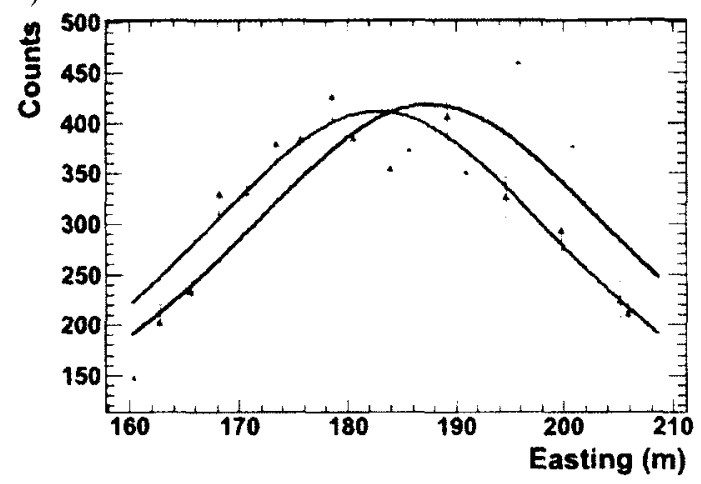

b)

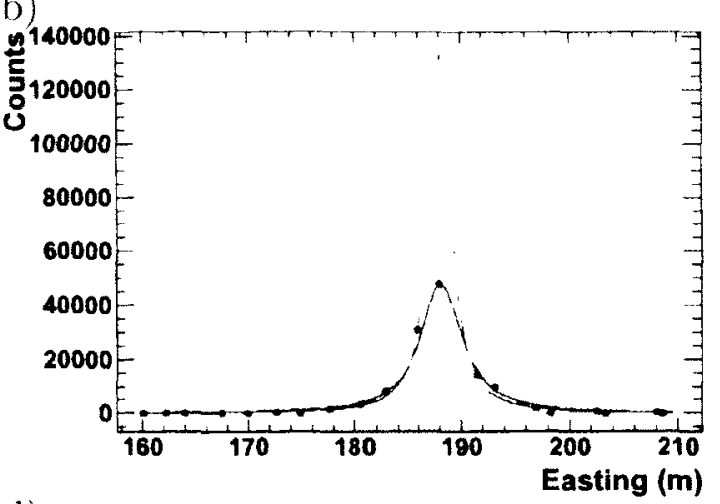

d)

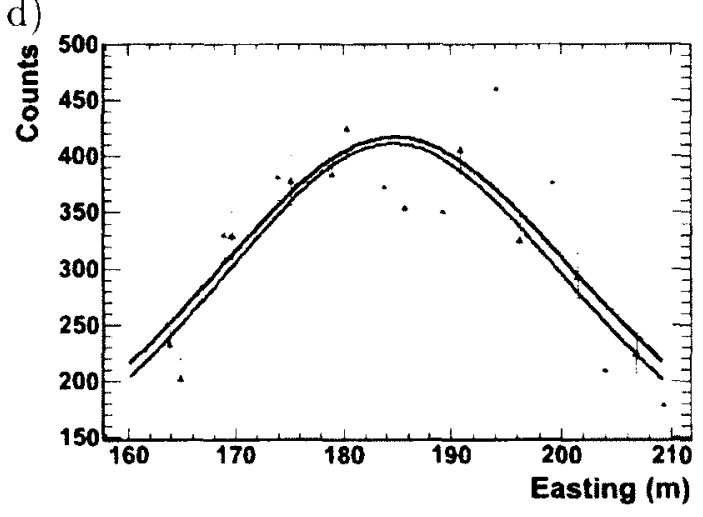

Figure 29: The counts profile for the Na-22 source for one pass in the energy window $450-550 \mathrm{keV}$ versus the position of the truck along the road at a) position A ( $2 \mathrm{~m}$ from the road), before the lag correction, b) position $\mathrm{A}$, after the lag correction was applied, c) position $\mathrm{C}$ ( $22 \mathrm{~m}$ from the road), before the lag correction and d) position $\mathrm{C}$, after the lag correction was applied. The curves represent tits to the counts profile - to be discussed in the next section.

has been applied, the two fitted curves for the two passes overlap as can be seen in Fig $29 \mathrm{~b}$ and Fig. 29d.

\subsection{Gross count method (GC)}

\subsubsection{Gross count plots for Na-22}

Background subtracted photopeak counts were plotted as a function of position for the Na-22 source for all positions. The highest photopeak is observed at position 
A $(2 \mathrm{~m})$, which is the closest position to the road. That means. the maximum counts are recorded when source is closest to the road. As the distance of the source from the road increases, the gross counts decrease sharply as necessitated by the inverse square law. Beyond position D $(32 \mathrm{~m})$, the count rate became insufficient for localization purposes. Hence, the positions $\mathrm{E}(42 \mathrm{~m})$ and $\mathrm{F}(52 \mathrm{~m})$ were not included in this analysis.

Fig. 30 shows the counts versus Easting profile for one pass, for the Na-22 source, at $20 \mathrm{~km} / \mathrm{h}$, for each position (A to D). Because the direction of motion of the truck was east-west, we use simply the easting measurement to represent the distance along the road. The measured data were fitted to the expression given by Eq. 9, and the results of the fit are overlaid on the data in Fig. 30. The distance of the source to the road, and the position of the source along the road, were extracted using the fit procedure. 
a)

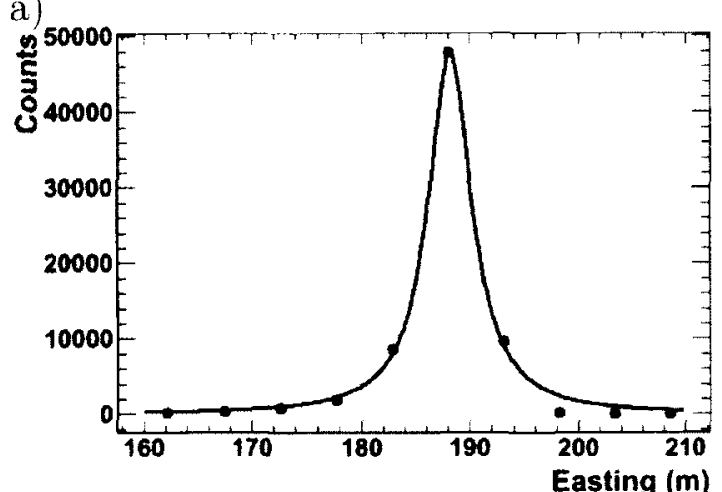

c)

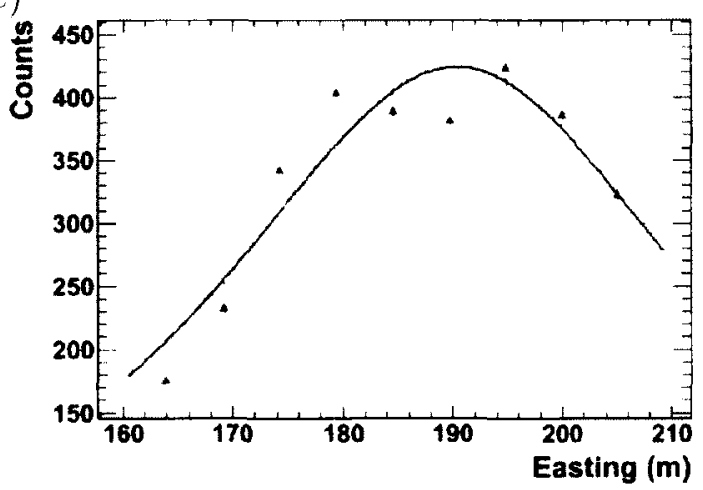

b)

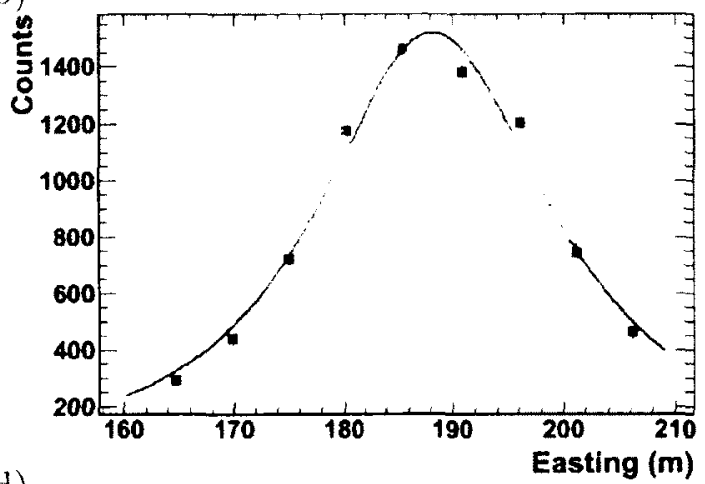

d)

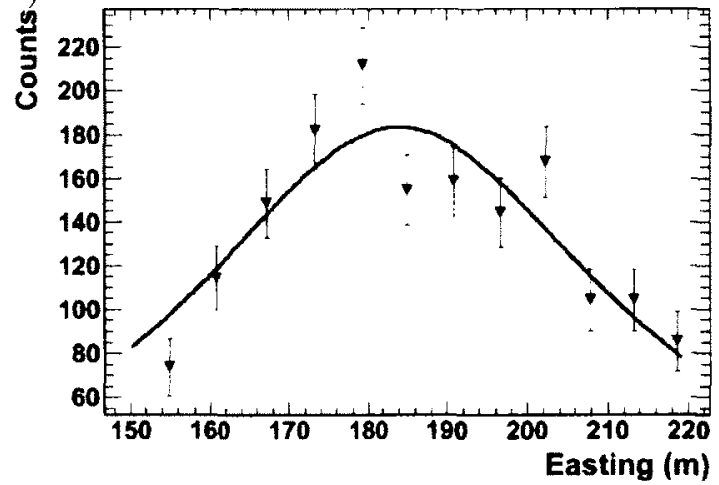

Figure 30: The counts profile versus the Easting for the $\mathrm{Na}-22$ source, at $20 \mathrm{~km} / \mathrm{h}$, for a) position $\mathrm{A}$, pass 3 , b) position $\mathrm{B}$, pass $3, \mathrm{c}$ ) position $\mathrm{C}$, pass 3 , and $\mathrm{d}$ ) position D, pass 3 . The curve shows the result of the fit of Eq. 9 to the counts profile. 
After measuring the source positions for all 16 passes, the reconstructed source positions were plotted, as shown in Fig. 31.
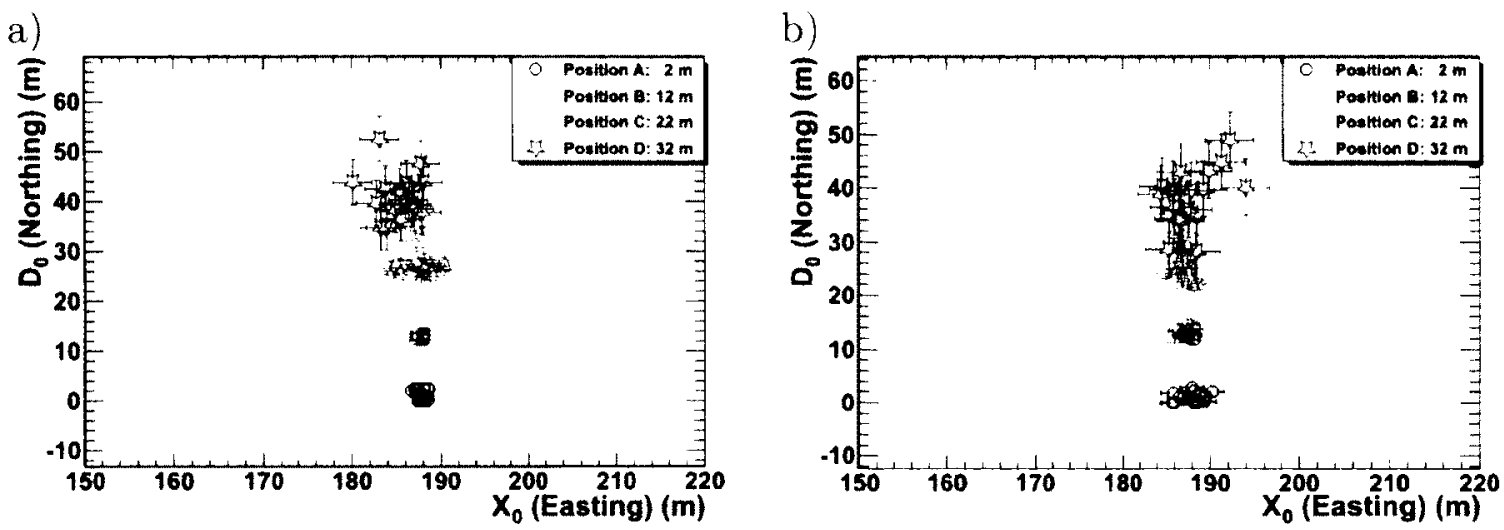

Figure 31: The reconstructed source positions (A to D) for 16 passes, for the $\mathrm{Na}-22$ source, shown for speeds of a) $20 \mathrm{~km} / \mathrm{h}$ and b) $40 \mathrm{~km} / \mathrm{h}$. The error bars represent the uncertainty on individual measurements as taken from the RMS deviation of the results over 16 passes.

Note that an additional offset of $55 \mathrm{~m}$ was applied to bring the Northing of the road to $0 \mathrm{~m}$. At $20 \mathrm{~km} / \mathrm{h}$, position $\mathrm{A}$ and $\mathrm{B}$ have been reconstructed well for 16 passes. Here, it needs to be mentioned that source positions $C$ and D are reconstructed in the sense that the results cluster at farther distances, as expected. However, there is a bias to farther distances. There is a shift in the larger-distance data (at position $\mathrm{C}$ and D) which we do not fully understand. The potential source of bias could be a residual lag effect or it could also be a residual effect of the detector's calibration.

The mean of the reconstructed source positions from 16 passes was taken for $\mathrm{Na}-22$ for each of the four positions (A to D), at 20 and $40 \mathrm{~km} / \mathrm{h}$, and plotted as shown in Fig 32 and Fig. 33. The mean was chosen to represent a typical individual measurement. The error bar which is shown is the RMS over sixteen passes, which represents the uncertainty on an individual measurement - not the error on the mean. The true source positions were obtained through a field survey using a Total Station (combination of traditional theodolyte and laser range finder). The results of these 
surveyed true source positions are also shown.

From Fig. 32, we see that as the source is moved farther from the road, the reconstructed source position also moves to longer distances. However. as the distance from the road is increased, a small deviation from the true source positions can be seen. For farther positions (C and D) or longer distances, the reconstructed source positions fall away from true source positions.
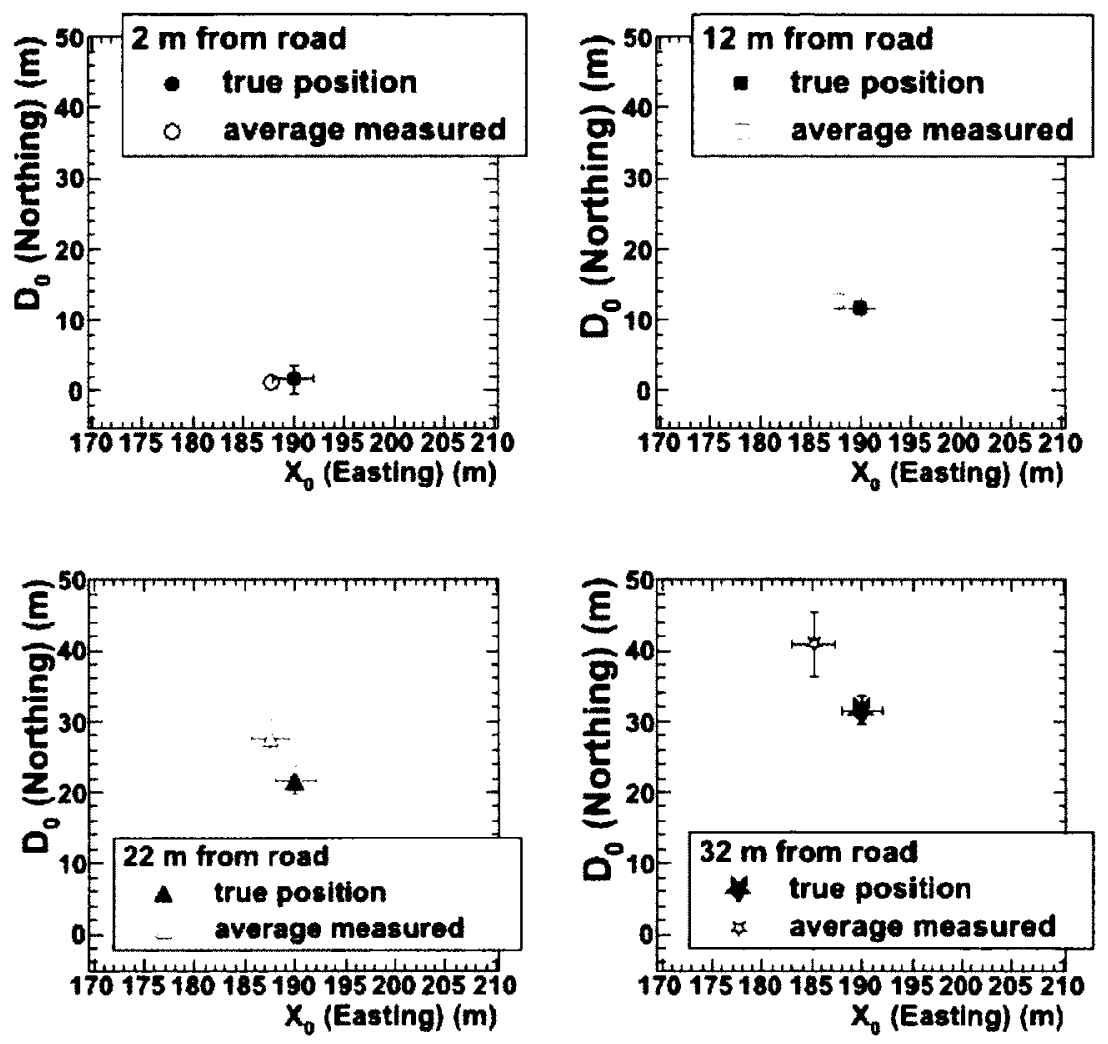

Figure 32: The mean of reconstructed source positions for 16 passes for position $\mathrm{A}$ ( $2 \mathrm{~m}$ from road), B (12 $\mathrm{m}$ from the road), C (22 $\mathrm{m}$ from the road) and D (32 $\mathrm{m}$ from the road), compared with true source positions, for $\mathrm{Na}-22$, at the speed of $20 \mathrm{~km} / \mathrm{h}$. The error bars show the RMS reconstructed position over 16 passes. 
Increasing the speed to $40 \mathrm{~km} / \mathrm{h}$. we are still able to recontruct the source position with a similar level of functionality, as shown in Fig. 33 .
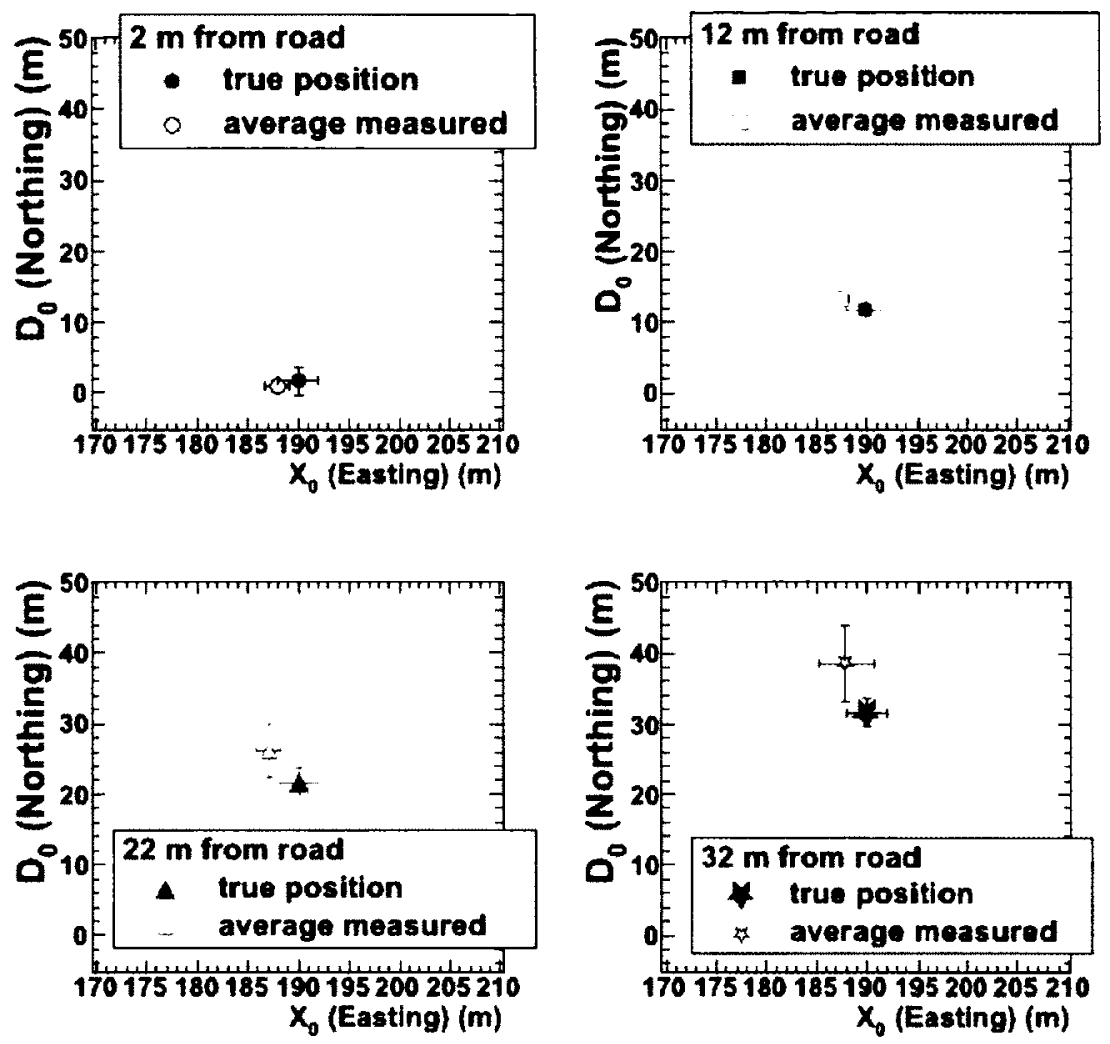

Figure 33: The mean of the reconstructed source positions for 16 passes for position A (2 $\mathrm{m}$ from road), B (12 $\mathrm{m}$ from the road), C (22 $\mathrm{m}$ from the road) and $\mathrm{D}$ (32 $\mathrm{m}$ from the road), compared with true source positions, for $\mathrm{Na}-22$, at the speed of $40 \mathrm{~km} / \mathrm{h}$. The error bars show the RMS reconstructed position over 16 passes. 
For $\mathrm{Na}-22$, at 20 and $40 \mathrm{~km} / \mathrm{h}$ speed, the mean of the extracted parameters from the fit are $\mathrm{N}, \mathrm{x}_{0}$ and $D_{0}$, summarized in Table 2 and Table 3 with the uncertainties in Easting $\left(\mathrm{RMS}_{\mathrm{X}_{0}}\right)$ and Northing $\left(\mathrm{RMS}_{D_{0}}\right)$. In the term $\mathrm{N}=\mathrm{S} \epsilon \mathrm{B}, \mathrm{S}$ is the source strength in Becquerel (Bq), $\epsilon$ is the efficiency for the detector, $B$ is the branching ratio for $\mathrm{Na}-22$. $D_{0}$ is the distance from the road in meters $(\mathrm{m})$ and $\mathrm{x}_{0}$ is the position along the road in meters $(\mathrm{m})$.

\begin{tabular}{lrrrrr}
\hline Position & $\mathrm{N}=\epsilon \mathrm{S}(\mathrm{Bq}) \mathrm{B}$ & $\mathrm{x}_{0}(\mathrm{~m})$ & $\mathrm{RMS}_{\mathrm{x}_{0}}(m)$ & $D_{0}(\mathrm{~m})$ & $\mathrm{RMS}_{D_{0}}(\mathrm{~m})$ \\
\hline $\mathrm{A}$ & $4.49 \times 10^{7}$ & 187.91 & 0.58 & 1.29 & 1.06 \\
$\mathrm{~B}$ & $3.69 \times 10^{7}$ & 187.72 & 0.44 & 12.83 & 0.43 \\
$\mathrm{C}$ & $5.04 \times 10^{7}$ & 187.43 & 1.92 & 27.56 & 2.57 \\
$\mathrm{D}$ & $4.96 \times 10^{7}$ & 185.38 & 2.14 & 41.02 & 4.59 \\
\hline
\end{tabular}

Table 2: For Na-22: at $20 \mathrm{~km} / \mathrm{h}$, the mean of the fit extracted results over sixteen passes for $\mathrm{N}, \mathrm{x}_{0}$ and $D_{0}$ with RMS deviations.

\begin{tabular}{lrrrrr}
\hline Position & $\mathrm{N}=\epsilon \mathrm{S}(\mathrm{Bq}) \mathrm{B}$ & $\mathrm{x}_{0}(\mathrm{~m})$ & $\mathrm{RMS}_{\mathrm{x}_{0}}(m)$ & $D_{0}(\mathrm{~m})$ & $\mathrm{RMS}_{D_{0}}(m)$ \\
\hline $\mathrm{A}$ & $7.73 \times 10^{7}$ & 188.32 & 1.27 & 0.92 & 0.95 \\
$\mathrm{~B}$ & $3.98 \times 10^{7}$ & 187.57 & 0.92 & 13.16 & 0.88 \\
$\mathrm{C}$ & $4.25 \times 10^{7}$ & 187.02 & 1.24 & 25.94 & 3.81 \\
$\mathrm{D}$ & $4.27 \times 10^{7}$ & 187.71 & 2.68 & 38.58 & 5.35 \\
\hline
\end{tabular}

Table 3: For $\mathrm{Na}-22$ : at $40 \mathrm{~km} / \mathrm{h}$, the mean of the fit extracted results over sixteen passes for $\mathrm{N}, \mathrm{x}_{0}$ and $D_{0}$ with RMS deviations.

Note that B for $511 \mathrm{keV}$ for $\mathrm{Na}-22$ is $170 \%$. We know that the Na-22 source strength in August of 2011 was $0.9 \mathrm{mCi}$ with an uncertainty $\sim 15 \%$. Therefore the effective intrinsic efficiency for the $511 \mathrm{keV}$ gammas was close to $100 \%$. This is understandable given that we have an $\sim 8^{\prime \prime}$ thickness of $\mathrm{NaI}(\mathrm{Tl})$.

We can say that the distance of the source to the road is approximated well at both speeds. 


\subsubsection{Gross count plots for Cs-137}

The same analysis was repeated for Cs-137. Background subtracted photopeak counts were plotted for the $\mathrm{Cs}-137$ source for all positions up to $\mathrm{C}$. Beyond position $\mathrm{C}$, the counts were insufficient for the analysis for this weaker source.

a)

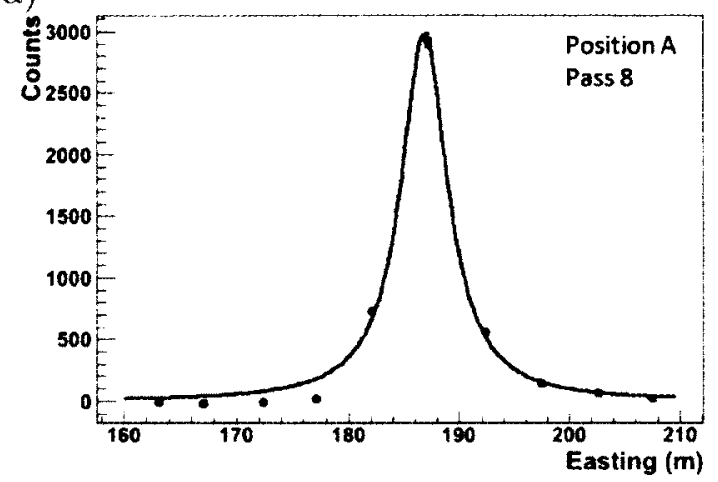

b)

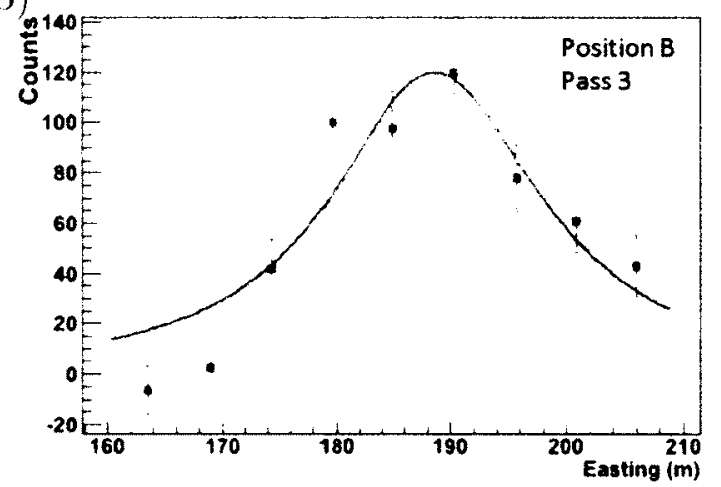

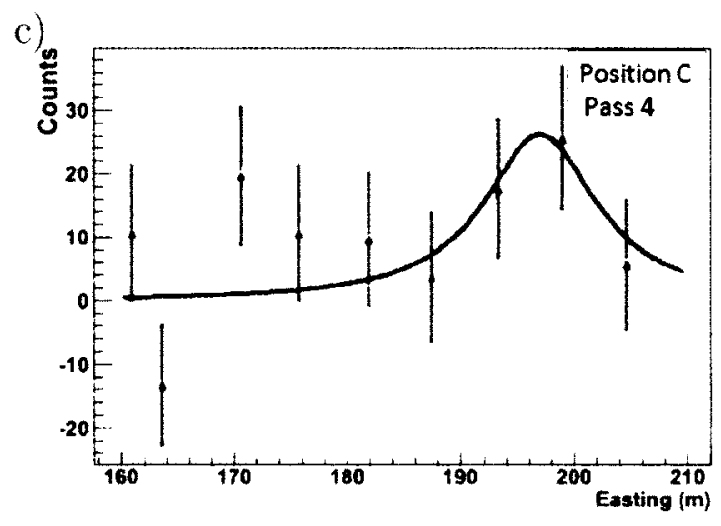

Figure 34: Gross counts in the energy window $550 \mathrm{keV}$ to $710 \mathrm{keV}$ for Cs-137 at $20 \mathrm{~km} / \mathrm{h}$ speed for a) position $A$ and pass $8, \mathrm{~b}$ ) position $B$ and pass 3 , and $\mathrm{c}$ ) position $\mathrm{C}$ and pass 4 , with the results of the fit to Eq. 9 overlaid. 
In similarity to the gross count method for Na-22. each data tile contained the information for 16 passes of the truck by the source. We had to extract the information for each pass. The measured data were then fitted to the expression given by Eq. 9 , and the distance of the source to the road was extracted along with the position on the road.

Curve fitting plots for each position up to position C. at $20 \mathrm{~km} / \mathrm{h}$, are shown in Fig. 34. The fitted data plots, again, show that the photopeak count rate drops sharply as the source moves away from the road.

Fig. 35 shows the mean of the reconstructed source positions for all passes. The near source positions have been reconstructed well particularly for position $\mathrm{B}$. There is a large uncertainty on the position along the road as well as on the distance from the road at position $\mathrm{C}$. This clearly shows that at this position, the count-rate becomes very small and farther distances would prove to be giving even more uncertainty for localization purposes. 

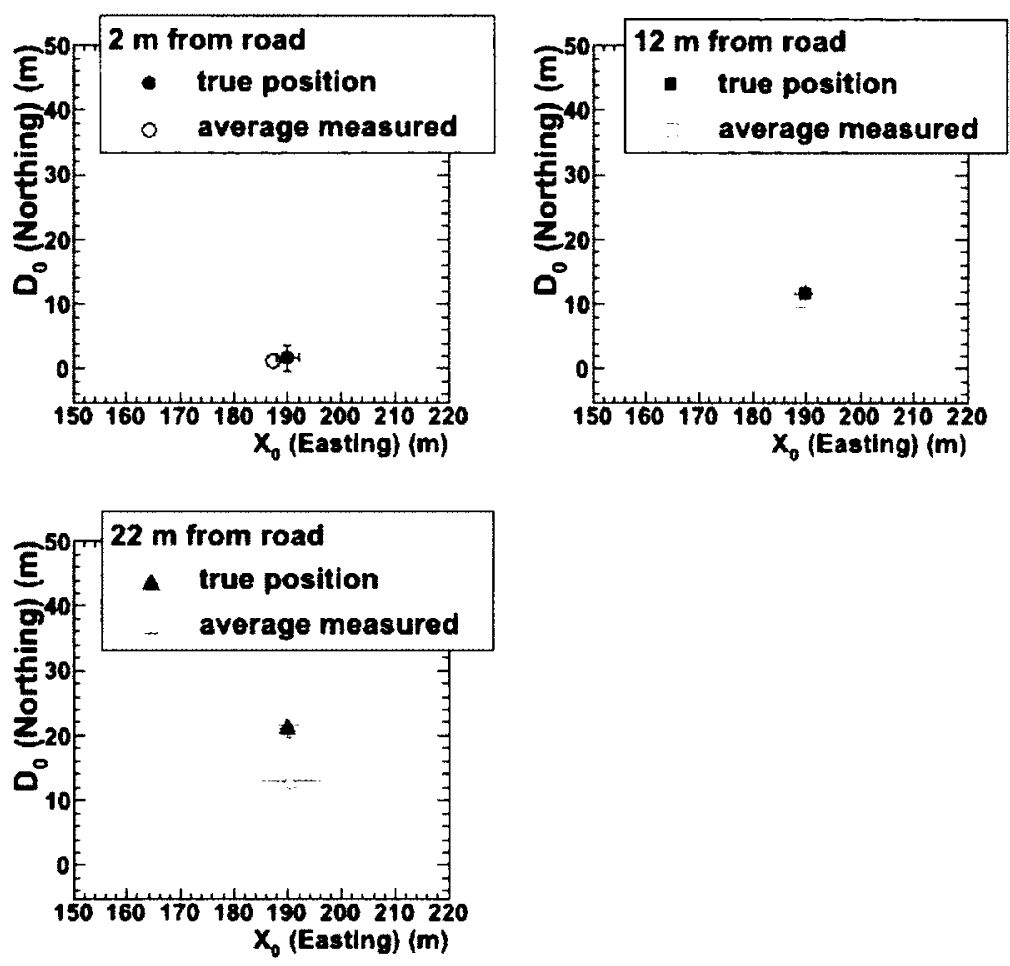

Figure 35: The mean of reconstructed source positions for position A ( $2 \mathrm{~m}$ from road), B (12 $\mathrm{m}$ from the road), C (22 $\mathrm{m}$ from the road), compared with true source positions, for Cs-137, at the speed of $20 \mathrm{~km} / \mathrm{h}$. The error bars show the RMS of the reconstructed positions over sixteen passes. 
The means of the fit extracted results over sixteen passes. for Cs-137 source up to position $\mathrm{C}$, at $20 \mathrm{~km} / \mathrm{h}$, are summarized in the Table 4 . which tell us that the source is localized well for near source positions. For farther source position C (22 m). there is a huge uncertainty on distance along the road and on the distance of the source from the road. This is due to the fact that the Cs-137 was a weaker source.

\begin{tabular}{lrrrrr}
\hline Position & $\mathrm{N}=\epsilon \mathrm{S}(\mathrm{Bq}) \mathrm{B}$ & $\mathrm{x}_{0}(\mathrm{~m})$ & $\mathrm{RMS}_{\mathrm{X}_{0}}(m)$ & $D_{0}(\mathrm{~m})$ & $\mathrm{RMS}_{D_{0}}(m)$ \\
\hline $\mathrm{A}$ & $3.41 \times 10^{6}$ & 187.05 & 0.62 & 2.67 & 0.98 \\
$\mathrm{~B}$ & $2.02 \times 10^{6}$ & 187.51 & 1.02 & 10.73 & 1.12 \\
$\mathrm{C}$ & $8.30 \times 10^{5}$ & 189.85 & 5.95 & 15.95 & 6.66 \\
\hline
\end{tabular}

Table 4: For Cs-137: at $20 \mathrm{~km} / \mathrm{h}$, the mean of the fit extracted results over 16 passes for $\mathrm{N}, \mathrm{x}_{0}$ and $D_{0}$ with RMS deviations.

In addition to being a weaker source, the branching ratio (B) for $662 \mathrm{keV}$ for Cs-137 is 85\%. We know that the Cs-137 source strength (S) in August of 2011 was $0.6 \mathrm{mCi}(0.02 \mathrm{GBq})$ with an uncertainty $\sim 15 \%$. Clearly, the averaged efficiency is very high.

\subsection{Directional method (DM)}

The directional method adds directionality to the count-rate based localization. The results consist of arrows, distance surface and subsequent reconstructed source position plots.

\subsubsection{Directional method plots for Na-22}

If the counts are very different from one side of the detector to the other, then the direction vector can be expected to be more significant, that is, it will be more likely to point in the direction of a source. In the present work, the arrow's length (counts 
based) is defined using Eq. 23. We use the inverse of the fractional uncertainty as a measure of the significance. Subsequently significance, $\xi$, is defined by Eq. 29 .

$$
\xi=\frac{|\vec{V}|}{\sqrt{\frac{u^{2} \cdot \sigma_{u}^{2}}{u^{2}+v^{2}}+\frac{v^{2} \cdot \sigma_{v}^{2}}{u^{2}+v^{2}}}}
$$

where $\sigma_{u}$ and $\sigma_{v}$ are statistical uncertainties on the components $\mathrm{u}$ and $\mathrm{v}$ which are based on counts.

The same data were analysed as for the gross count method. The relative total counts from four detectors, during a truck survey past the Na-22 source for all positions were obtained. Natural background was suppressed by including only those counts arising from the $511 \mathrm{keV}$ photopeak (a spectral window from $450 \mathrm{keV}$ to $550 \mathrm{keV}$ ) in the sums, $C_{i}$. The vector $\vec{V}$ was calculated and positions were determined, following the method outlined in Chapter 4.

\section{Directional method, Na-22 source, driving at $20 \mathrm{~km} / \mathrm{h}$}

The significance for each position was measured and plotted as shown in Fig. 36 . 

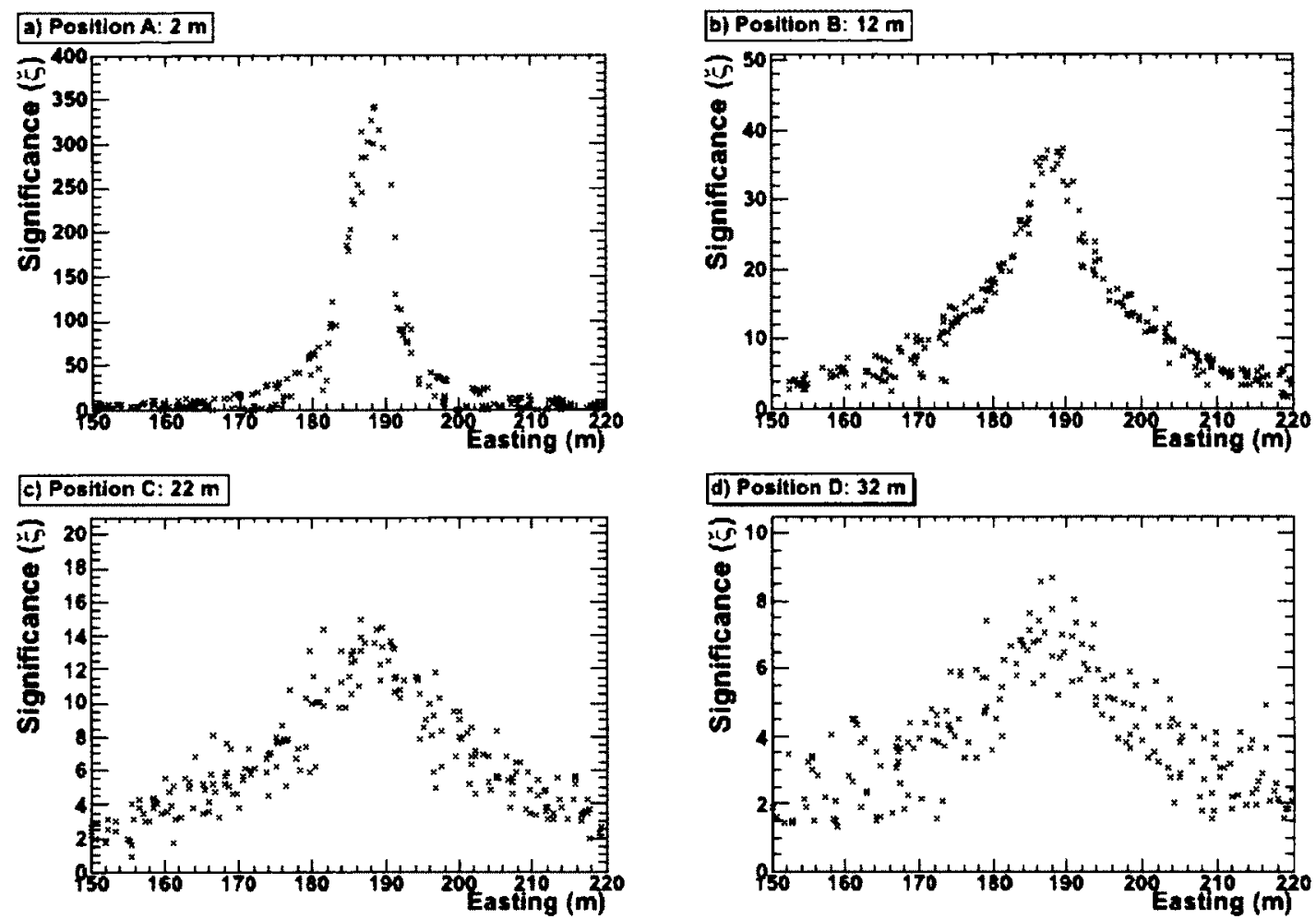

Figure 36: The measure of significance (Eq. 29) for source positions a) A, $2 \mathrm{~m}$ from the road b) B, $12 \mathrm{~m}$ from the road c) C, $22 \mathrm{~m}$ from the road and d) D, $32 \mathrm{~m}$ from the road. All passes are shown. 
The significance at position $\mathrm{A}$ is highest. as expected. At other positions the measure of significance results are better but the significance is reduced as the distance from the road has increased. Significance cut offs to reject or accept direction vectors (arrows) were introduced and the value of 3.5 was used.

a)

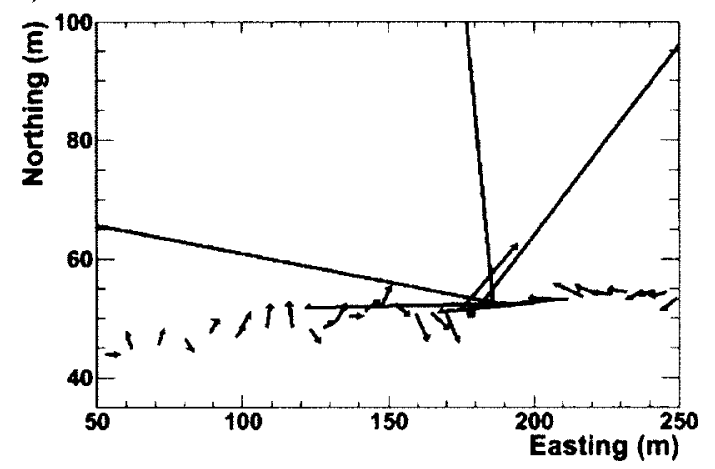

c)

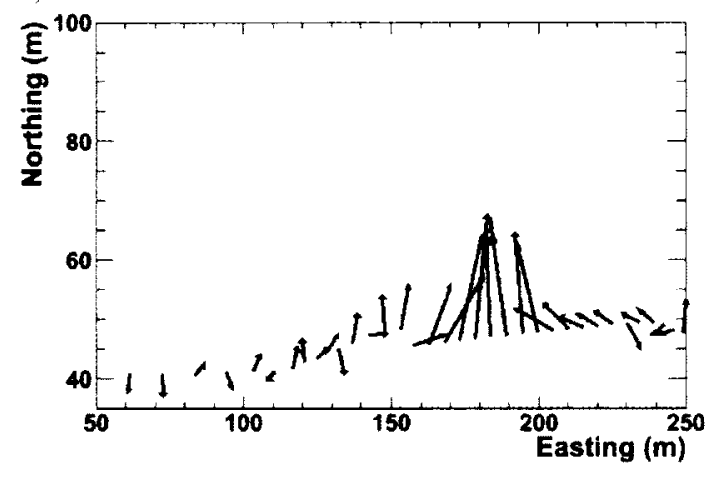

b)

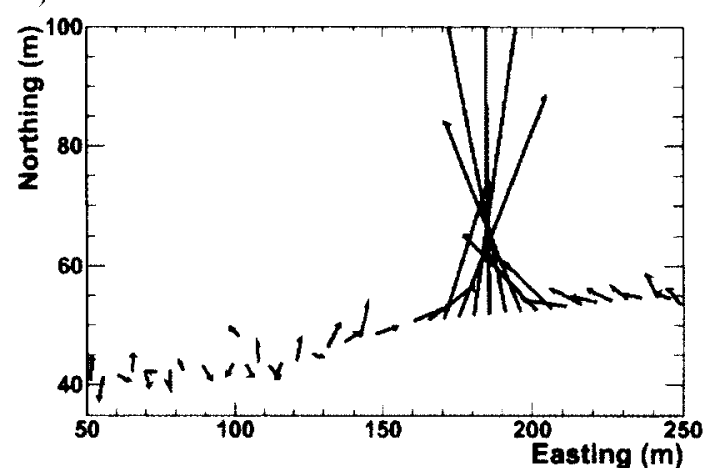

d)

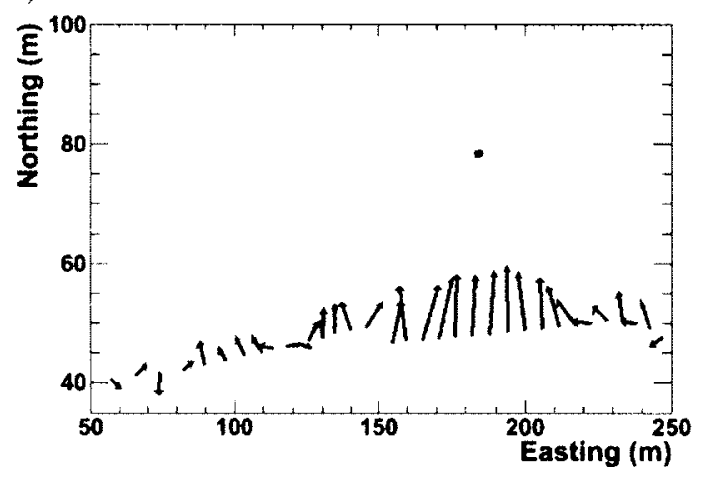

Figure 37: The direction arrows for one pass are shown versus position along the road for the $\mathrm{Na}-22$ source at $20 \mathrm{~km} / \mathrm{h}$, for the positions a) A, $2 \mathrm{~m}$ from the road, b) B, $12 \mathrm{~m}$ from the road, c) C, $22 \mathrm{~m}$ from the road and d) D, $32 \mathrm{~m}$ from the road. The reconstructed source position is given by the dot.

Fig. 37 shows the arrows pointing towards the probable source location for one pass, for each source location, at the truck's speed of $20 \mathrm{~km} / \mathrm{h}$. On either side of the plots, away from the closest approach to the source, natural background dominates and the arrows are small and point in random directions. As the truck passes by the source, maximal counts are observed, and the arrows are longer and point toward the source location. 
Note that for optimal display of the arrow's intersection length some of the longest arrows are cut off at the edge of the Fig. 37a and Fig. 37b.

Fig. 38 shows the distance function $D(x, y)$, given by Eq. 27, for one pass at $20 \mathrm{~km} / \mathrm{h}$ for positions $\mathrm{A}$ to $\mathrm{D}$. The distance function $\mathrm{D}(\mathrm{x}, \mathrm{y})$ varies smoothly on both axes. The minimum of the surface plot is obtained by minimizing algorithm. It has a single minimum as shown in Fig. 38. The dot represents that minimum, which is the optimum source location.
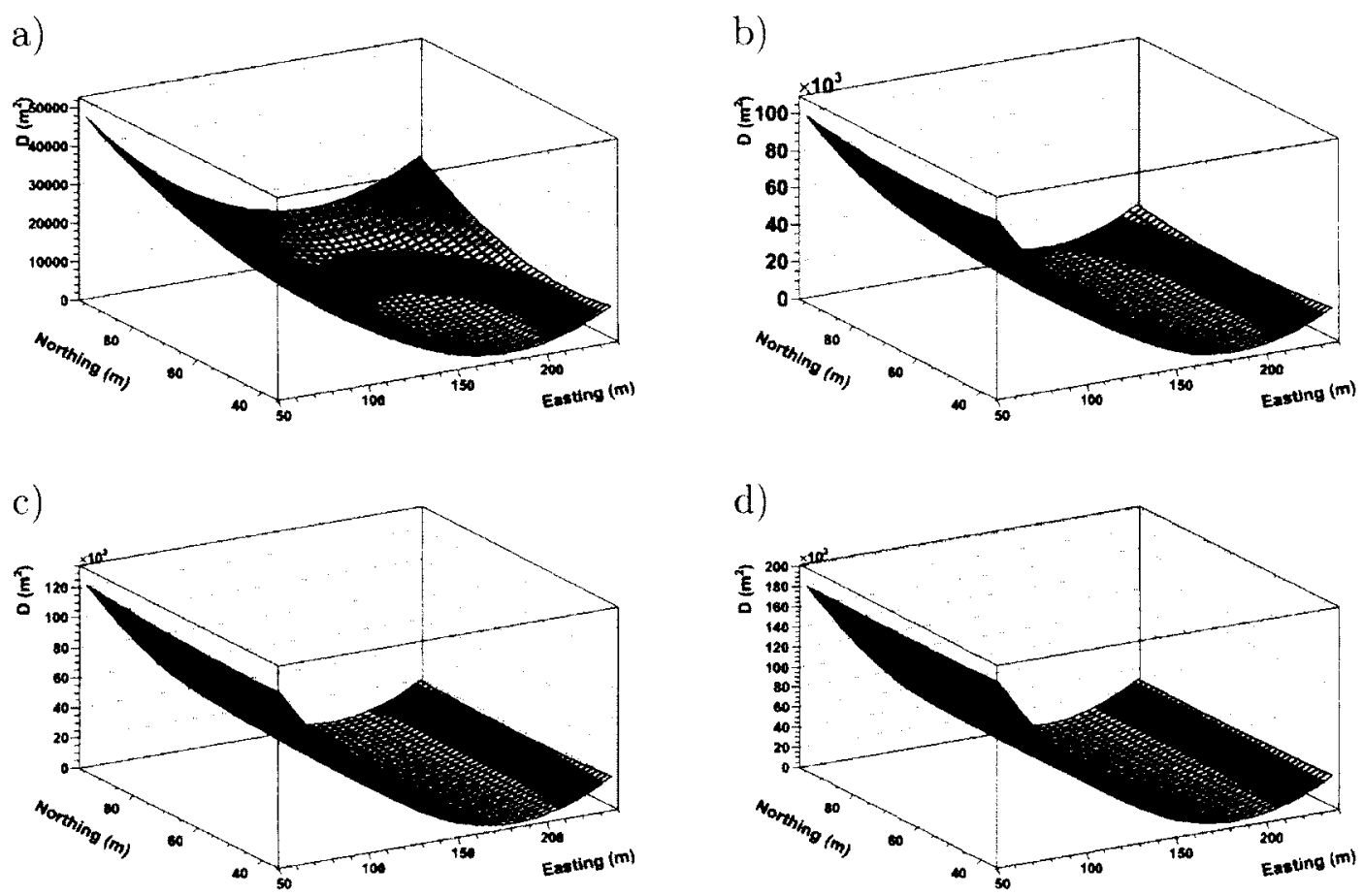

Figure 38: The distance function $\mathrm{D}(\mathrm{x}, \mathrm{y})$ versus Easting and Northing for the Na-22 source at $20 \mathrm{~km} / \mathrm{h}$, giving the measured source location, for one pass for the positions a) A, $2 \mathrm{~m}$ from the road, b) B, $12 \mathrm{~m}$ from the road c) C, $22 \mathrm{~m}$ from the road, d) D, $32 \mathrm{~m}$ from the road. The reconstructed source position is given by the dot. 
As with the gross count method, the measured reconstructed source positions for 16 passes, in the directional method were plotted. for the data at $20 \mathrm{~km} / \mathrm{h}$ speed, as shown in Fig. 39. It can be seen that the source positions are reconstructed reasonably well for near sources. As the distance increases, the distribution of reconstructed source positions show a spread.

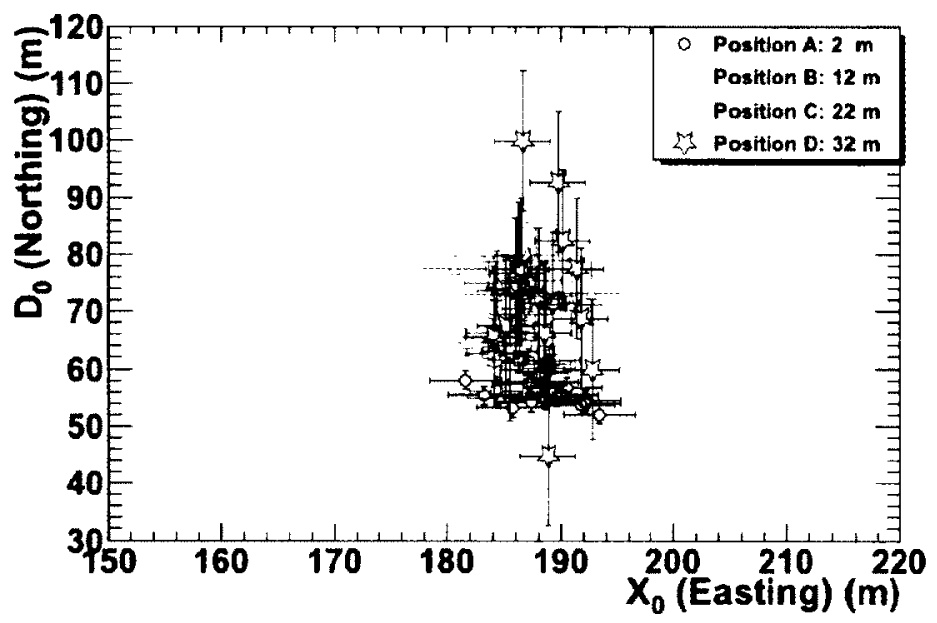

Figure 39: The measured reconstructed source positions for 16 passes for the source positions $A$ ( $2 \mathrm{~m}$ from the road), B (12 $\mathrm{m}$ from the road), C (22 $\mathrm{m}$ from the road) and $D(32 \mathrm{~m}$ from the road), at $20 \mathrm{~km} / \mathrm{h}$.

The mean of the measured reconstructed source positions for 16 passes was determined. These reconstructed source positions were then compared to the true source positions, as shown in Fig 40. The error bars represent the RMS of the distribution. 

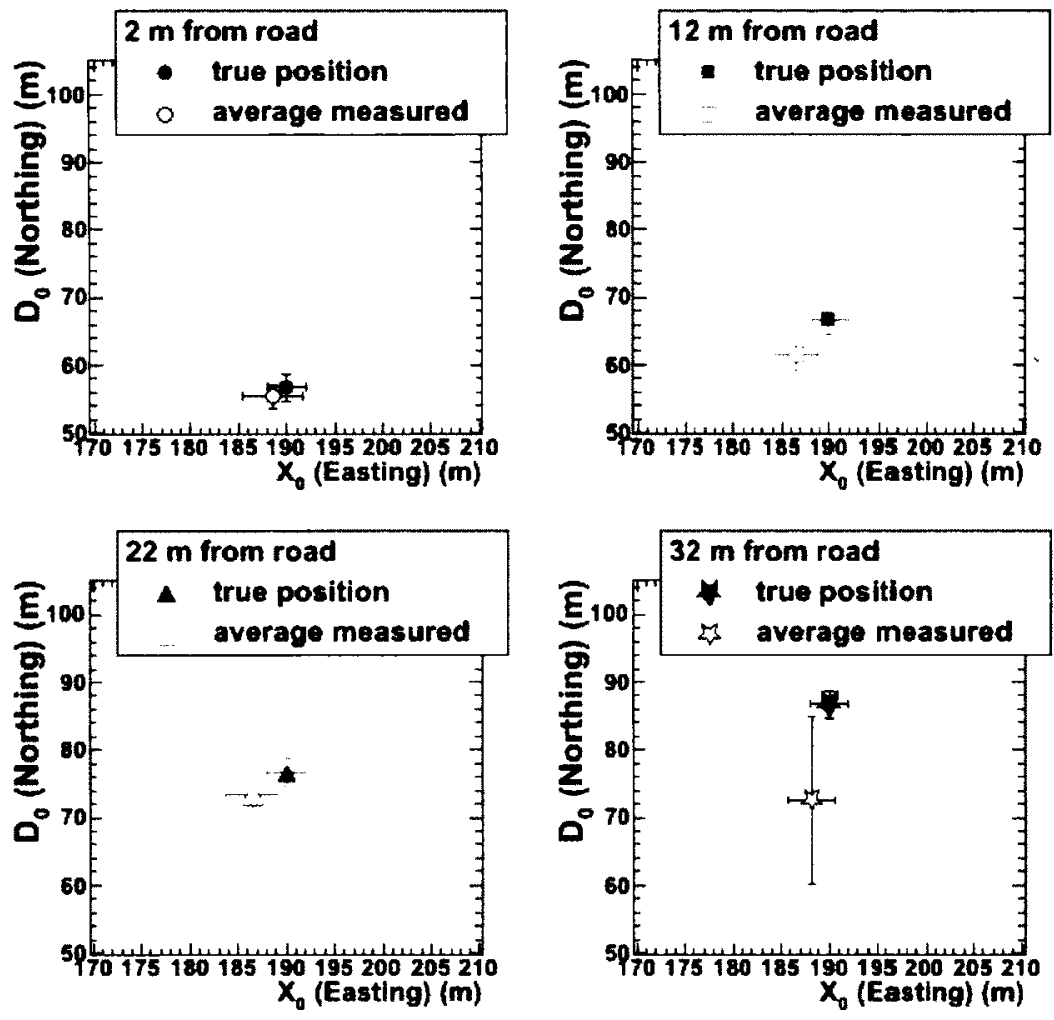

Figure 40: The mean of reconstructed source positions with the true source positions for position $\mathrm{A}$ ( $2 \mathrm{~m}$ from the road), position $\mathrm{B}$ ( $12 \mathrm{~m}$ from the road), position $\mathrm{C}$ (22 $\mathrm{m}$ from the road) and position $\mathrm{D}$ (32 $\mathrm{m}$ from the road), at $20 \mathrm{~km} / \mathrm{h}$, for $\mathrm{Na}-22$ source. The error bars show the RMS of the distributions of reconstructed source positions. 
At near distances, the spread of recontructed positions along the road is larger than the spread of the reconstructed positions perpendicular to the road. However, at larger distances, the spread of reconstructed positions along the road is smaller than the spread of reconstructed positions perpendicular to the road. Fig. 41 explains the reasons for this.

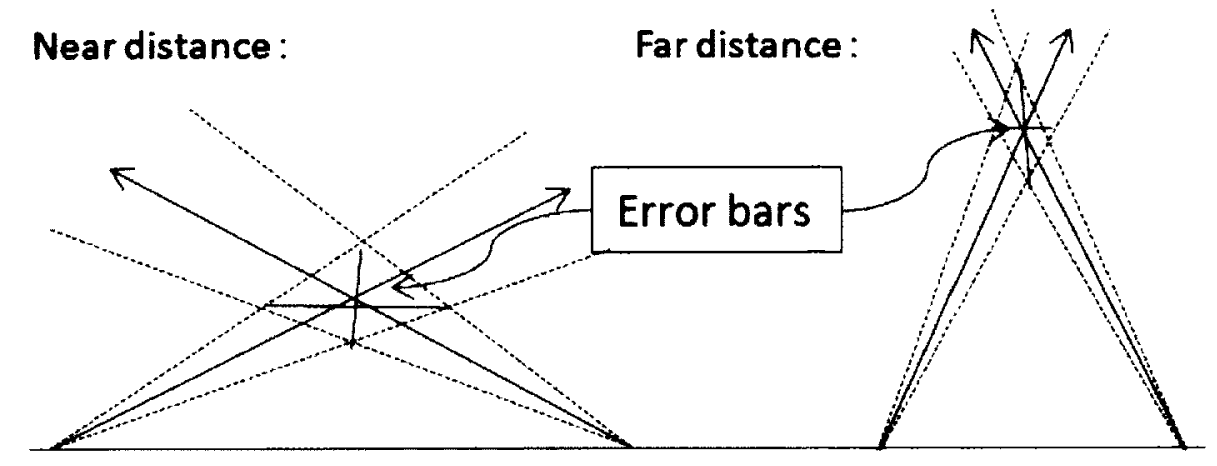

Figure 41: A demonstration of the error on position along the road and perpendicular from the road, assuming a roughly constant angular uncertainty for the measurements of both near and far sources. At near distances, the error along the road is large and perpendicular distance small. At far distances, vice versa.

At near distances, the arrows are nearly parallel to each other, pointing toward each other, and nearly parallel to the road. This results in the uncertainty on their crossing point being large along the road, and small perpendicular to the road. At far distances, the arrows are again nearly parallel to each other, pointing in the same direction, and perpendicular to the road. This results in the error on the distance along the road being small and on the distance from the road being large. The errors at large distance are also increased by the decreased statistical precision. 
Table 5, summarizes the means of the measured distances from the road as well as the positions on the road along with the deviations (uncertainties).

\begin{tabular}{lrrrr}
\hline Position & $\mathrm{x}_{0}(\mathrm{~m})$ & RMS $_{\mathrm{X}_{0}}(m)$ & $D_{0}(\mathrm{~m})$ & RMS $_{D_{0}}(m)$ \\
\hline $\mathrm{A}$ & 188.61 & 4.9 & 2.30 & 1.71 \\
$\mathrm{~B}$ & 186.56 & 3.05 & 11.10 & 2.39 \\
$\mathrm{C}$ & 186.58 & 5.66 & 22.21 & 5.66 \\
$\mathrm{D}$ & 184.49 & 12.37 & 39.89 & 12.37 \\
\hline
\end{tabular}

Table 5: For Na-22, at $20 \mathrm{~km} / \mathrm{h}$, the means of the measured values of $\mathrm{x}_{0}, D_{0}$ with RMS deviations.

\section{Directional method, Na-22 source, driving at $40 \mathrm{~km} / \mathrm{h}$}

There were a smaller number of arrows measured at $40 \mathrm{~km} / \mathrm{h}$ because the truck moves more quickly past the source. The direction vectors and surface plots are shown for select passes. Where there was an insufficient number of measurement positions for the minimization to work, the reconstructed source position could not be obtained.

Fig. 42 shows the direction vectors for the data collected at $40 \mathrm{~km} / \mathrm{h}$, for one pass. Where background dominates at the sides of the plot the arrows point in random directions. Where the signal dominates the count-rate, the arrows point toward the source location. Here again, the dot represents the optimum source location determined. 
a)

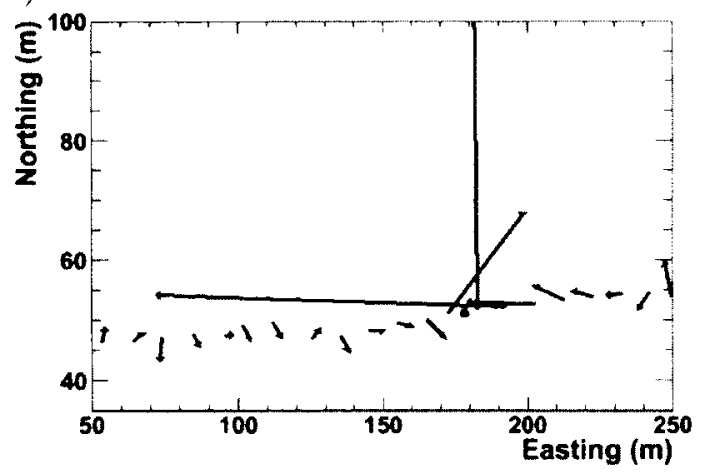

c)

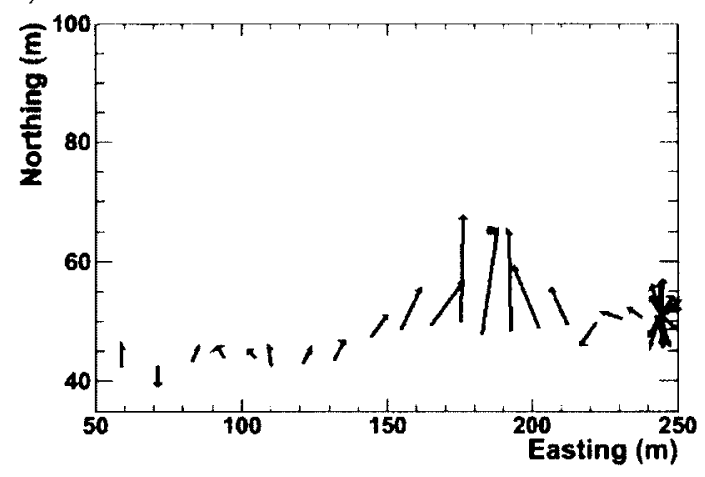

b)

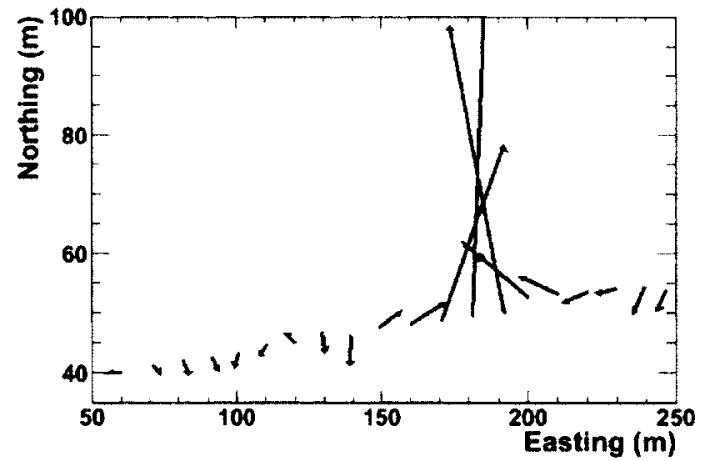

d)

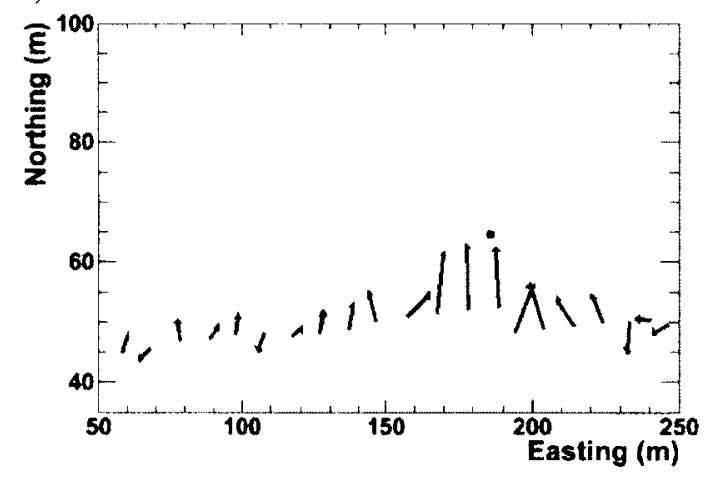

Figure 42: The direction arrows for one pass are shown versus position along the road for the $\mathrm{Na}-22$ source at $40 \mathrm{~km} / \mathrm{h}$, for the positions a) A, $2 \mathrm{~m}$ from the road, b) B, $12 \mathrm{~m}$ from the road, c) C, $22 \mathrm{~m}$ from the road, d) D, $32 \mathrm{~m}$ from the road. The reconstructed source position is given by the dot. For optimal display of the arrow's intersection length some of the longest arrows are cut off at the edge of a) and b). 
Fig. 43 shows the distance function $D(x, y)$ and the single mimimum of it. Again. the minimization of sum of the squares distance function gave the optimum source location. The same Eq. 27, as in $20 \mathrm{~km} / \mathrm{h}$ case, was miminized using Minuit [5].
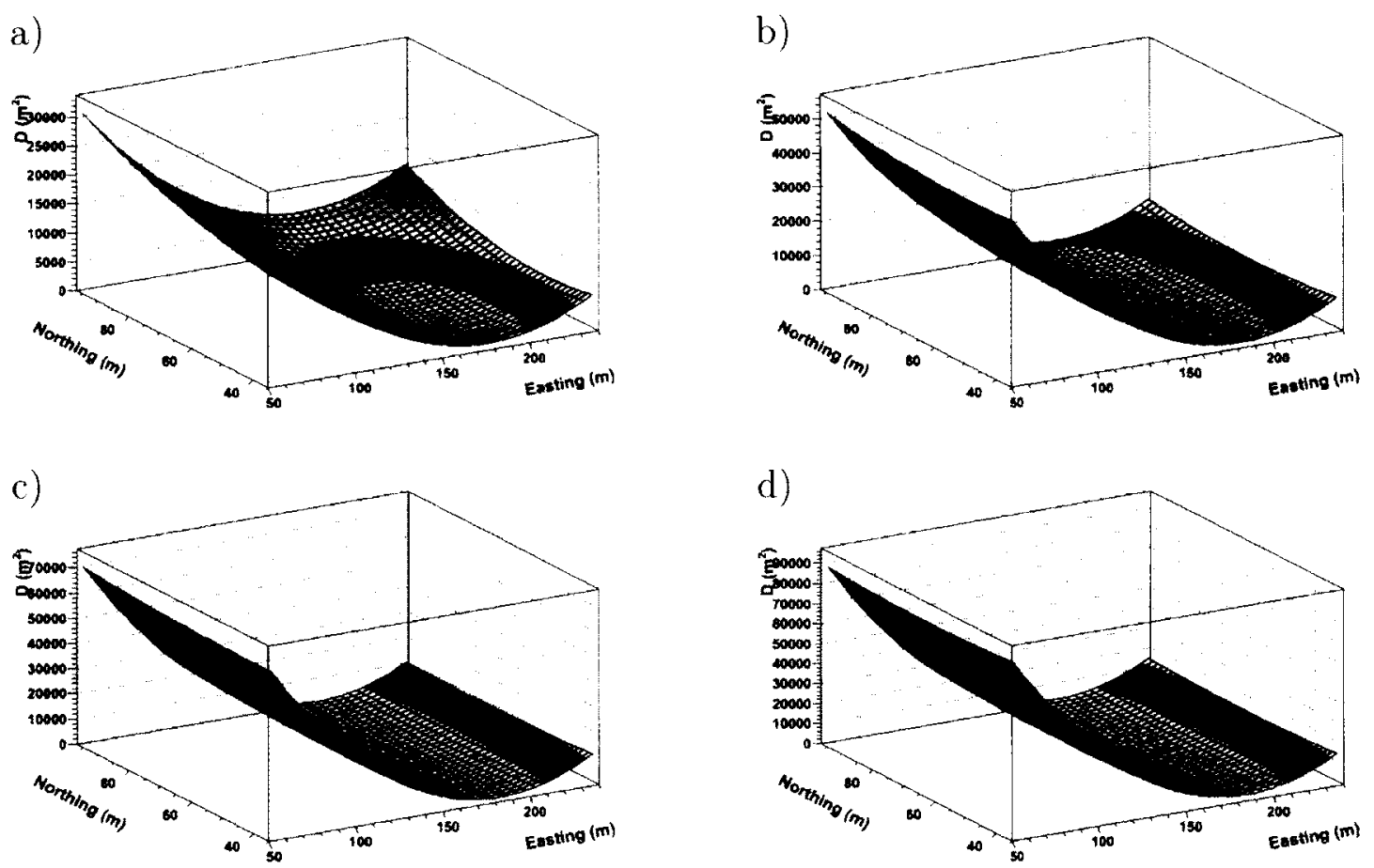

Figure 43: The distance function $\mathrm{D}(\mathrm{x}, \mathrm{y})$ versus Easting and Northing for Na-22 source at $40 \mathrm{~km} / \mathrm{h}$, giving optimum source location, for one pass for positions a) A, $2 \mathrm{~m}$ from the road b) B, $12 \mathrm{~m}$ from the road c) C, $22 \mathrm{~m}$ from the road d) D, $32 \mathrm{~m}$ from the road. The reconstructed source position is given by the dot. 
At $40 \mathrm{~km} / \mathrm{h}$. for many passes the number of measurements was insufficient for the directional method to converge. Without a sufficient number of position measurements converging. we do not have an estimate of the uncertainty on reconstructed source positions. Therefore, we do not present reconstructed source positions plots for $40 \mathrm{~km} / \mathrm{h}$.

\subsubsection{Directional method plots for Cs-137}

The Cs-137 source was much weaker than the Na-22 source. This meant that the statistical significance of the measurements was much lower. Fig. 44 shows the directional significance for Cs-137.
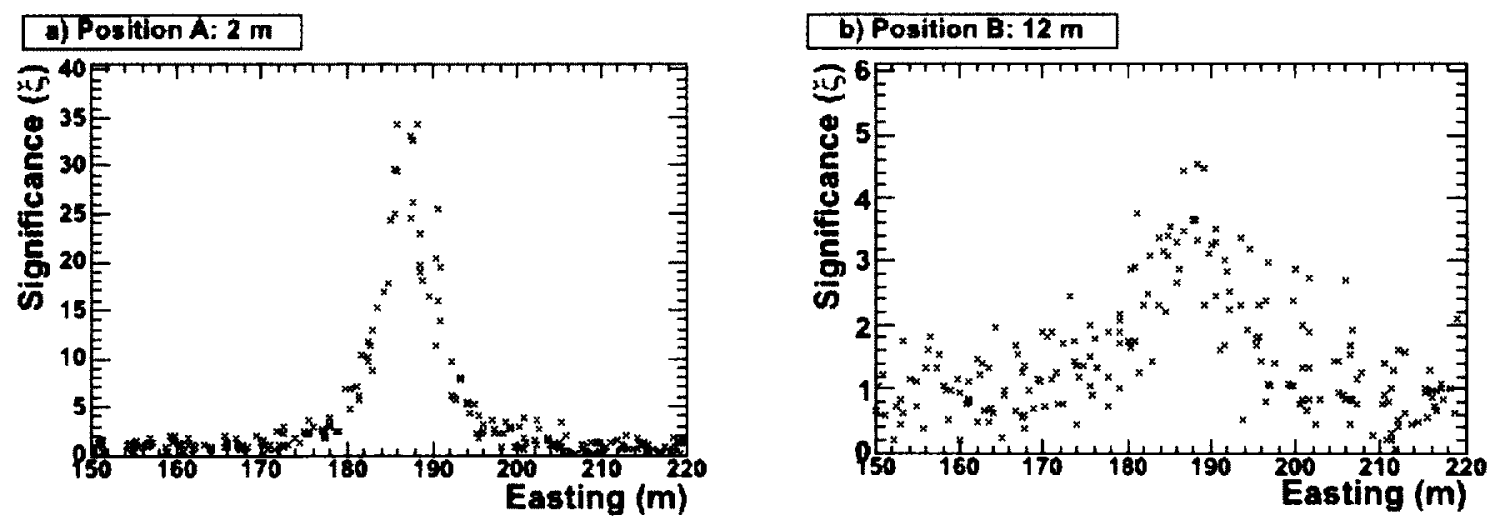

Figure 44: The measure of significance for Cs-137 at position a) A, $2 \mathrm{~m}$ from the road and b) B, at $12 \mathrm{~m}$ from the road.

Different significance cut-offs were tested. If the significance cut-off is set too high, then the arrows' significance is high and they would point towards the source, however, there would be a small number of arrows. If the significance cut-off is set too low, then a lot of arrows that do not point towards the source would be accepted and the localization would fail.

Fig. 45a and Fig. 45b show the reconstructed source positions for Cs-137 at position $\mathrm{A}$ and $\mathrm{B}$ with significance cut-off set at 1.8 and for position $\mathrm{A}$ set at 3.5 
a)

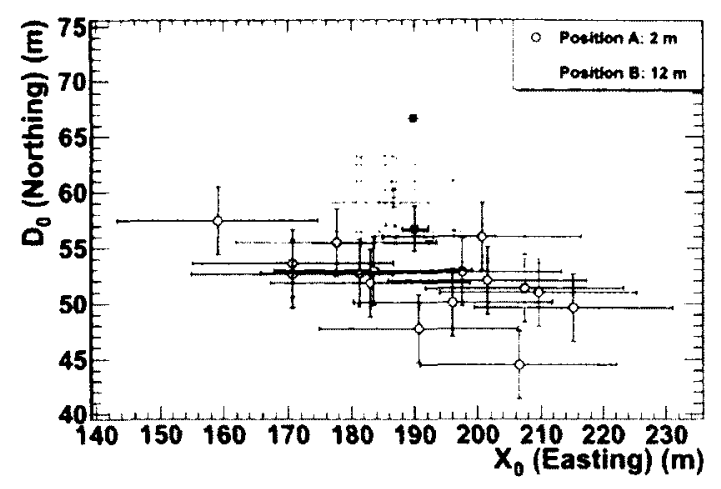

b)

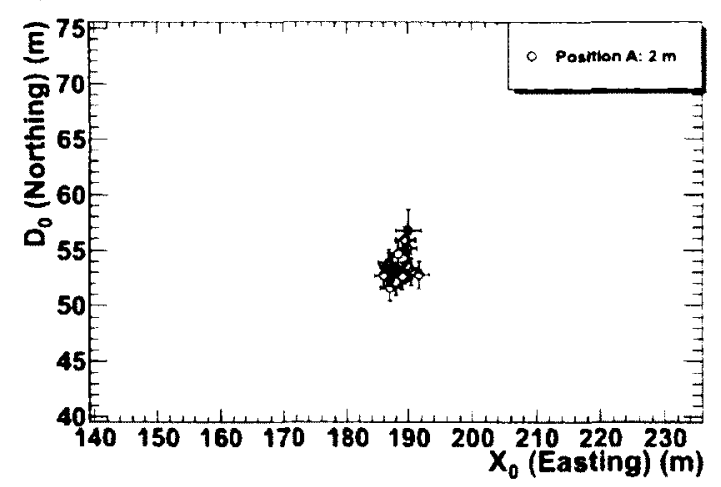

Figure 45: The reconstructed source positions for Cs-137, for 16 passes, at $20 \mathrm{~km} / \mathrm{h}$, at position $A$ and $B$ with a) significance $(\xi)$ cut-off set at 1.8 and b) significance $(\xi)$ cut-off set at 3.5 at position $\mathrm{A}$. The true positions are represented by filled markers.

respectively. The reconstructed source positions are just too far or random compared to the true source positions especially at the low cut-off value of 1.8 . With the low cut-off value of 1.8 , the reconstructed source positions do not compare well with the true source positions. With the high cut-off value of 3.5 , an insufficient number of arrows were available for the reconstruction for all positions except the closest one, position $A$.

Thus, the gross count method can reconstruct the source position and distance from the road for the weak source, while the directional method can not.

\subsection{Angular resolution measure}

The angular resolution of the whole system of the detector in motion can be charaterised using an angular resolution measure. The angular resolution measure includes, for example, the uncertainty due to the location of the vehicle. An average angular resolution of $\sim 3$ degrees was obtained for the directional system alone. as discussed in Chapter 4's directional calibration section. However, in the outdoor 


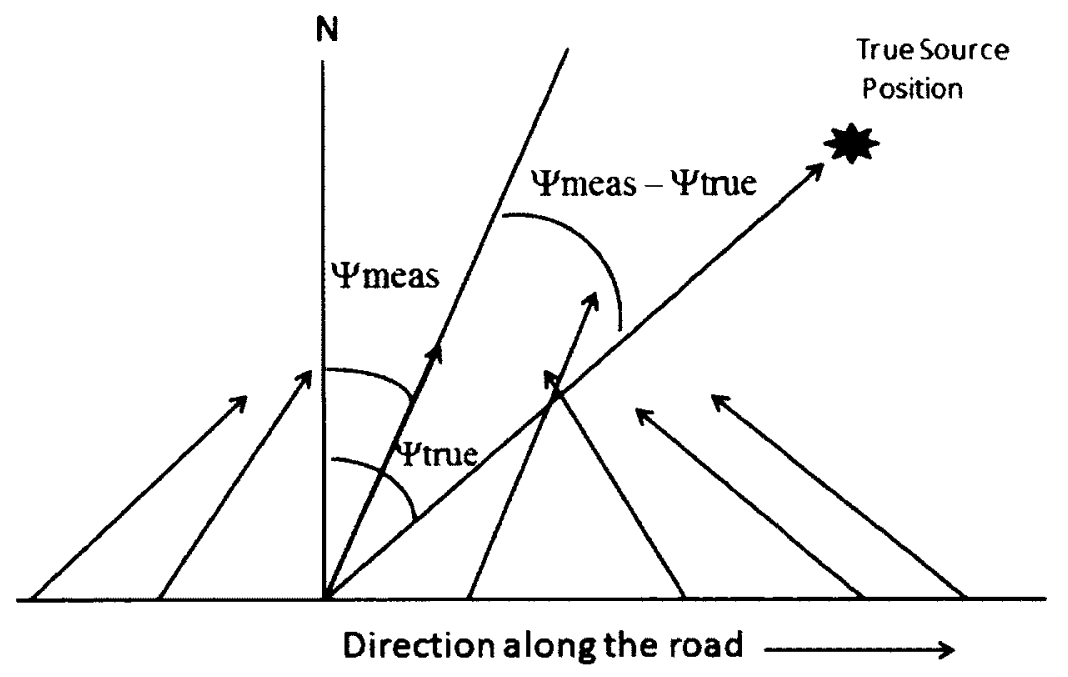

Figure 46: Schematic of the measured angle ( $\Psi_{\text {meas }}$ ) of the arrows with respect to the North along with angle difference $\left(\Psi_{\text {meas }}-\Psi_{\text {true }}\right)$ to the true source position.

situation with the detector in motion, the effective angular resolution is much worse.

The angles subtended by the arrows with the North ( $\left.\Psi_{\text {meas }}\right)$ direction as well as the angles between North and the line between the measured truck's position and the true source position ( $\left.\Psi_{\text {true }}\right)$, were measured as represented in Fig. 46.

The distribution of $\Psi_{\text {meas }}-\Psi_{\text {true }}$ is shown in Fig. 47 for all passes at $20 \mathrm{~km} / \mathrm{h}$ for the Na-22 source at positions A through D. 
a)

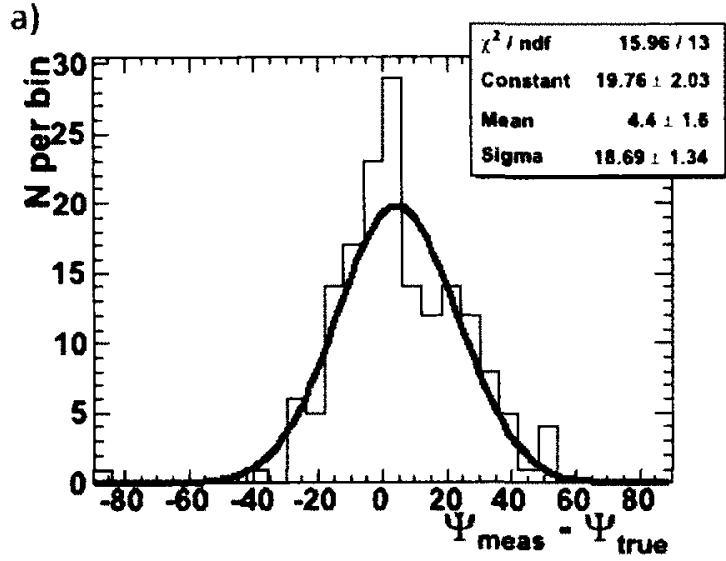

c)

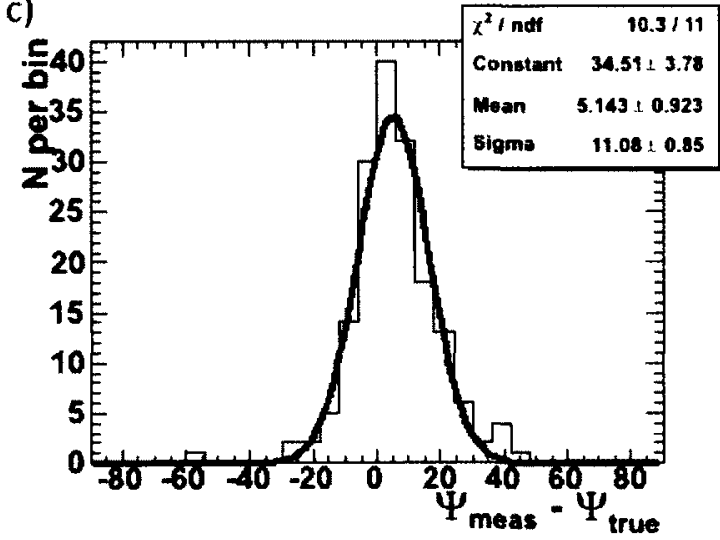

b)

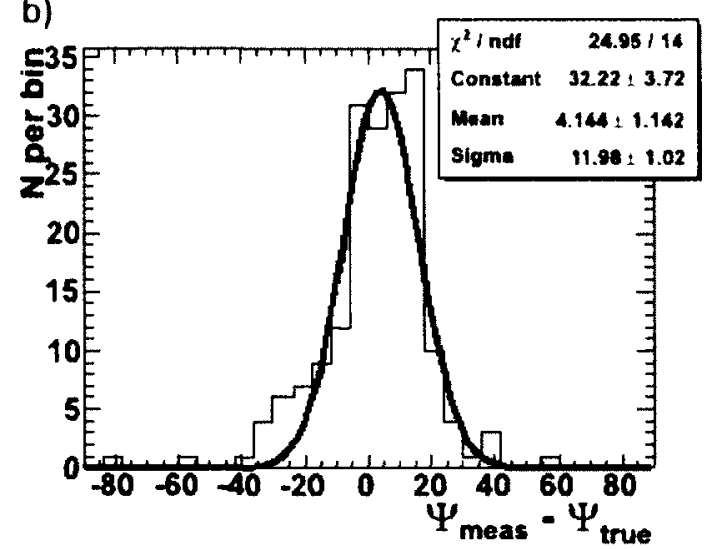

d)

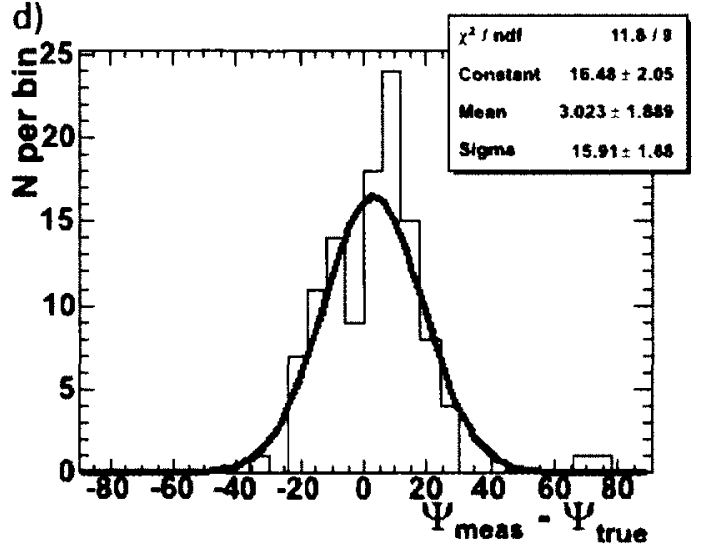

Figure 47: The angular resolution measure derived from the arrows distribution and the true source distribution for positions a) A, $2 \mathrm{~m}$ from the road, b) B, $12 \mathrm{~m}$ from the road, c) C, $22 \mathrm{~m}$ from the road, and d) D, $32 \mathrm{~m}$ from the road, fitted with a Gaussian. 
These distributions have been fit with a Gaussian distribution and the spread of the distributions, $\sigma$, is taken as the angular resolution measure. We find that the angular resolution measure varies from $\sim 11^{0}$ to $\sim 19^{\circ}$. It is significantly worse than the detector's angular resolution of $\sim 3^{0}$. Therefore, we can expect that improvements in other aspects of the measurements such as the measurement of the truck's position, could significantly improve the directional method.

\subsection{Comparison}

In the gross count method, the source positions are reconstructed correctly within uncertainties characterized by the RMS deviations (error bars) over 16 passes. For near source positions (position $A$ and $B$ ), the reconstructed source positions are measured best. The RMS width is around $1 \mathrm{~m}$, over 16 passes, in this case. For farther sources (position $\mathrm{C}$ and position D), the precision degrades to approximately $5 \mathrm{~m}$ due to statistical fluctuations in the count-rate. The precision on distance from the road $\left(D_{0}\right)$ and the precision on the position along the road $\left(x_{0}\right)$, degrades more (up to $10 \mathrm{~m}$ ).

In the directional method, taking into consideration the uncertainty on the position measurements given by the RMS deviation over sixteen passes, the reconstructed source positions correspond reasonably well to the true source positions. For near sources (position A), the precision on position on the $\operatorname{road}\left(x_{0}\right)$, is largest $(6 \mathrm{~m})$. The precision on Northing or distance from the road $\left(D_{0}\right)$, is around $4 \mathrm{~m}$. For far sources, the precision on position along the road $\left(x_{0}\right)$ reduces to approximately $5 \mathrm{~m}$. The precision on distance from the road $\left(D_{0}\right)$ degrades to its worst value, that is, around $25 \mathrm{~m}$. 


\section{Chapter 6}

\section{Discussion and conclusions}

Two methods are presented in this work to determine the location of a source at some distance from the road. Both methods have some merits which will be discussed in the following.

\subsection{Gross count method}

An analytic solution based on the inverse square law, with attenuation in air (see Eq. 9), was implemented to reconstruct source positions using the measurement data collected at different speeds and at four different distances of the source from the road. Fig. 32 and Fig. 33, showed that the reconstructed source positions are measured well, though with some spread from the true positions. The expected behaviour for the gross count method at a higher speed where there are fewer measurements would be to reduce the accuracy of the localization. However, with the speed increased from $20 \mathrm{~km} / \mathrm{h}$ to $40 \mathrm{~km} / \mathrm{h}$, there was no loss of precision and accuracy.

The uncertainty increases with the distance of the sources from the road, as expected from the loss of statistics. Systematic sources of uncertainty include,

- Non-isotropic source: Isotropic sources radiate in all directions uniformly throughout $4 \pi$ geometry. A non-isotropic source would not emit gammas 
uniformly in all directions as possibly in the present case.

- Background subtraction method: Background subtraction is important in any analysis. In the present work, the mean of the background counts was subtracted from the measured data. A better way to do this might have been to subtract background which depends on position.

- Non-isotropic detector acceptance: a function of the angle, that is. $\epsilon(\theta, \Phi)$. An efficiency function is required, which is a function of the angle around the detector. This can be achieved through Monte Carlo study and laboratory validation. This is beyond the scope of the present work.

- Uncorrected lag: The spectrum was placed at a location half a second back from where it was tagged. However, if the counts increase non-linearly, a more sophisticated procedure should have been used.

The results indicate an offset slightly to the left of the true source locations. This is probably a systematic uncertainty in the true results, which could come from non-optimal locations of the satellites in the sky, atmospheric disturbances of the GPS signal, or a limitation in the conversion from the Cartesian coordinates (ECEF) to UTM coordinates on the earth's surface.

Overall, the method provides a good spatial localization. In previous works, an accuracy of $\sim 15 \mathrm{~m}$ was achieved. It can be said that the present study achieved a better estimation (fewer meters) in source localization with some bias. The problem with the (inverse-square-law-based) gross count method is that it can not pinpoint the direction to the location of the source in real-time unless some directional capability is added. The directional method was developed to address this issue. 


\subsection{Directional Method}

This method is based on the measurement of the angle between the North direction and the line from the truck's position to the source. This angle is a function of the position of the truck. The method converges to results which are reasonable in that the uncertainty is small when the source location is close and it increases as the true distance from the road to the source increases.

A small uncertainty along the road was observed but there was a large uncertainty in the distance from the road, for sources at longer distances. Fig. 37d and Fig 42d showed that for the source at large distance, the directional vectors are all nearly orthogonal to the road (and therefore nearly parallel to each other), so there is a large uncertainty in where they cross.

Likewise for the dataset at $2 \mathrm{~m}$, although the uncertainty is very reasonable, the uncertainty on the distance along the road is larger than it is on the distance from the road. This is because the directional vectors are near parallel to each other (and pointing in opposite directions) along the road.

In the case of the Cs-137 source, for many passes the directional method did not converge due to the small number of statistics because of it being a weaker source and having a much lower branching ratio (85\%) compared to Na-22 (170\%). A stronger source may have given better results as in case of $\mathrm{Na}-22$, discussed above.

The offset of all of the measured results slightly to the left of the true location is likely due to a systematic mismeasurement of the true location, as discussed in the previous section. However, there is also a slight bias which is most apparent for the data at position $\mathrm{B}$ (at $12 \mathrm{~m}$ from the road) wherein the reconstructed positions tend to underestimate the distance to the source. This could be a limitation of the method, which uses an ad hoc estimator for the directional vector. If a better function for the correction of the measured direction could be found, the method might be improved. 
The sources of systematic uncertainty include.

- Resolving truck's position: The uncertainties associated with the measurement of the truck's position, discussed in the previous section. also affect the directional method.

- Background subtraction: An implicit assumption was made that the background has no directionality in the directional method. If the background has directionality, it may still be having an effect.

- Uncorrected lag: The measured truck's position may have been tagged wrongly due to the lag effect. The lag was corrected for but a better method maybe needed, as discussed in previous section.

- Detector's geometry: The angle of the source was estimated reasonably by the directional detector using arctangent of the differences in the count-rates, though, uncertainties in the measured angle from the true angle could be caused by the geometry of the detector. However, the detector geometry meant that there were large uncertainties in the measured angle for some measured angles for which we were unable to correct. A better correction to the angles as determined by this method, or a better initial estimation of these angles, could improve the directional method.

Overall, the method provides reasonable source localization especially at short distances.

\subsection{Conclusion}

Both the gross count and directional methods have reconstructed source positions reasonably well along the road and at a distance from the road, with an uncertainty 
of a few meters for a source strength of around $\sim 1 \mathrm{mCi}$ situated within $32 \mathrm{~m}$ of the road.

The gross count method turned out to be a better method for localizing near sources and gave very good precision for farther sources.

The directional method that was implemented did not provide an advantage in the precision of the measurements for source localization. However, the directional method does have several other advantages. For example, it does not require a separate background survey, it indicates the side of the road where the source is located, and it provides a real-time direction to the source.

\subsection{Outlook}

Source localization is a complex problem as there are many techniques available and choices have to be made. The results rely heavily on the choices made for the source strength, the vehicle's speed, the detector's efficiency, the algorithms developed and how they take care of the background. The sources used, Na-22 and Cs-137, were not very strong. The strength of the $\mathrm{Na}-22$ source was $0.9 \mathrm{mCi}$ and that of Cs-137

$0.6 \mathrm{mCi}$. Speeds of $20 \mathrm{~km} / \mathrm{h}$ and $40 \mathrm{~km} / \mathrm{h}$ were chosen as is the common practice in gamma survey spectrometry, though, other speeds could have been chosen. The detector's efficiency depends on many things such as the kind of crystals used and the geometry of the crystals. The way the methods are implemented could also have an effect.

This project can provide a basis for further source localization techniques both in scope and scale. Different scenarios and conditions can also be implemented and enhanced. For example, the sources involved in this project were not shielded. Future work should consider a situation where sources are shielded. Further, in the present study, the sources were stationary, while in numerous applications, the sources could 
be moving.

Finally, in this project, the sources were localized one at a time. However, in practice there is a crucial need to detect and localize multiple sources at once. especially in threat detection applications, where the prime concern is the national security.

The methods described here are off-line methods, which means that they are used during post processing of the data. These methods would be most useful for scientific research and analysis at an advanced level. The methods could be adjusted to provide real-time applications, where the localization of the sources could be performed in real time (on-line methods). The enhancements needed in this case include the consideration of all the scenarios mentioned above, and the addition of a graphical user interface to make it more user friendly. 


\section{Appendix A}

\section{Gross Count Method}

//Some functions and plotting

//GC - the fitting expression used and Gross count curves

Double_t GCFunction(Double_t $x$, Double_t $S$, Double_t X_0, Double_t D_0)

$\left\{\right.$ Double_t dist $=x-X_{-} 0 ;$ Double_t $R 2=$ dist $*$ dist $+D_{-} 0 * D_{-} 0$;

Double_t $f=S * A * \operatorname{epsilon} * \exp (-1$. $*$ Lambda*sqrt $(R 2)) /\left(4 * M \_P I * R 2\right)$;

return $f$;

\}

void GrosscountSS2ToMinimize(Int_t \&npar, Double_t* gin, Double_t \&f, Double_t* par, Int_t iflag) $\{$ Double_t $x, g$; Double_t ss2 =0; TArrow* arrow;

for (int $j=$ ArrowInfo. Ind $1 ; j<=$ ArrowInfo. Ind $2 ; j++)\{$

arrow $=($ TArrow $*$ ) Arrows $\cdot A t(j) ; x=\operatorname{arrow}->\operatorname{GetX1}()$;

$\mathrm{g}=\operatorname{GCFunction}(\mathrm{x}, \operatorname{par}[0], \operatorname{par}[1], \operatorname{par}[2]) ;$

ss $2+=(g-G r o s s C o u n t s[j]) *(g-G r o s s C o u n t s[j]) ;\} f=s s 2 ; \operatorname{return} ;\}$

void FindGrosscountCurve(int dataset, int pass)

\{if (pass $==0)\{/ /$ set indices of the arrows of a given dataset

ArrowInfo. Ind1 $=$ ArrowInfo.Pass [dataset -1$][0]$. Ind1;

ArrowInfo.Ind2 = ArrowInfo.Pass[dataset -1$]$ [ArrowInfo.Pass [dataset-1]. size ()-1]. Ind2; 
\} else $\{/ /$ set indices of the arrows of a given dataset/pass ArrowInfo.Ind1 $=$ ArrowInfo.Pass [dataset-1] [pass-1].Ind1; ArrowInfo.Ind2 $=$ ArrowInfo.Pass [dataset-1] [pass-1].Ind $2 ;\}$ // get starting values for $X_{-} 0$ and $D_{-} 0$ (mode of grosscounts) Double_t $D_{-} 0=0$; Double_t $X_{-} 0=0$; TArrow* arrow; for (int $j=$ ArrowInfo. Ind $1 ; j<=$ ArrowInfo. Ind $2 ; j++$ ) \{ if (GrossCounts $\left.[j]>D_{-} \_\right)\left\{D_{-} 0=\right.$ GrossCounts $[j]$; arrow $=($ TArrow $*)$ Arrows.$\left.\left.A t(j) ; X \_0=1+\operatorname{arrow}->\operatorname{GetX1}() ;\right\}\right\}$ TMinuit $*$ ptMinuit $=$ new $\operatorname{TMinuit}(3) ; / / 3$ parameters: $S, X_{-} 0, D_{-} 0$ ptMinuit->SetPrintLevel(0); if (GrosscountMinimization==1) ptMinuit->SetFCN (GrosscountChi2ToMinimize) ; if (GrosscountMinimization==2) ptMinuit->SetFCN(GrosscountSS2ToMinimize); Double_t arglist [10];

Int_t ierflg $=0 ; \operatorname{arglist}[0]=1$;

ptMinuit->mnexcm("SET ERR", arglist,1,ierflg);

// starting values and step sizes for parameters static Double_t vstart [3] $=\left\{2000000, X_{-} 0,30\right\}$; static Double_t step $[3]=\{1,0.1,0.1\}$; ptMinuit->mnparm(0, "S", vstart[0], step [0], 0, 0, ierflg); ptMinuit->mnparm(1, "X_0", vstart [1] , step [1] , 0, 0, ierflg); ptMinuit->mnparm(2, "D", vstart [2], step [2] , 0, 0, ierflg); // peform minimization $\operatorname{arglist}[0]=500$; $\operatorname{arglist}[1]=1.0$; ptMinuit->mnexcm ("MIGRAD" , arglist, 3, ierflg); double fParamValo, fParamVal1, fParamVal2; double fParamErro,fParamErr1,fParamErr2; ptMinuit->GetParameter (0,fParamVa10,fParamErr0); 
ptMinuit->GetParameter (1,fParamVal1,fParamErr1);

ptMinuit->GetParameter (2,fParamVal2,fParamErr2);

if (pass $==0)\{$ TGrosscountParameters params;

params $. S=f$ ParamValo; params $\cdot X_{-} 0=f$ ParamVal1;

params.D_O = abs (fParamVal2);ArrowInfo.Grosscount.push_back (params);

\} else \{ArrowInfo.Pass[dataset-1] [pass-1].S = fParamVal0;

ArrowInfo.Pass [dataset-1] [pass-1] .X_0 = fParamVal1;

ArrowInfo.Pass [dataset-1] [pass-1].D_0 = abs (fParamVal2);

\}$\}$

void FindAllGrosscountCurves() ffor (int $i=0 ; i<$ ArrowInfo. Ndataset; $i++$ ) \{

FindGrosscountCurve $(i+1,0)$;

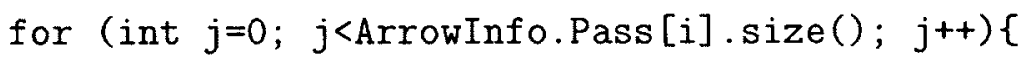

FindGrosscountCurve $(i+1, j+1) ;\}\}\}$

//Plotting Functions for passes at any Position from A to D

void DrawGrosscountPass(int dataset, int pass)

$\{$ int $\mathrm{n} 1=$ ArrowInfo.Pass[dataset-1] [0]. Ind1;

int N2 = ArrowInfo.Pass [dataset-1] [ArrowInfo.Pass [dataset-1]. $\operatorname{size}()-1]$. Ind2;

Double_t ymin $=0$; Double_t ymax $=0$;

TArrow* arrow;

arrow $=($ TArrow $*)$ Arrows. At (n1);Double_t xmin $=\operatorname{arrow}->\operatorname{GetX1}()$;

Double_t $x \max =\operatorname{arrow}->\operatorname{GetX1}()$;

for (int $i=n 1 ; i<=N 2 ; i++)\{$ arrow $=$ (TArrow*) Arrows.At $(i)$;

Double_t $x=\operatorname{arrow}->\operatorname{GetX1}()$; if $(x<\operatorname{xmin})\{x \operatorname{xmin}=x$;

else $\{$ if $(x>x \max )\{x \max =x ;\}\}$

Double_t $c=$ GrossCounts $[i]$; if $(c>y \max )\{\operatorname{ymax}=c ;\}$

\} // draw grosscounts 
char $s[50] ;$ sprintf ( $s, " G r o s s$ count, set $\% s$, pass $\% d "$, ArrowInfo. Names [dataset-1].c_str(), pass);

TCanvas *c1=new $\operatorname{TCanvas}(s, s)$;

gPad->SetLeftMargin(0.15); gPad->SetBottomMargin(0.15);

TMultiGraph *mg $=$ new TMultiGraph(); Double_t $x[200], y[200]$;

Double_t step $=(x \max -x \min ) / 200$; for (Int_t $i=0 ; i<200 ; i++)\{$

$x[i]=x \min +i *$ step;

$\mathrm{y}[\mathrm{i}]=$ GCFunction $(\mathrm{x}[\mathrm{i}]$, ArrowInfo.Pass [dataset-1] [pass-1].S,

ArrowInfo.Pass [dataset-1] [pass-1] . X_0,

ArrowInfo.Pass [dataset-1] [pass-1].D_0);

TGraph *gr1 = new $\operatorname{TGraph}(200, \mathrm{x}, \mathrm{y})$;

gr1->SetLineColor(dataset); gr1->SetLineWidth (3);

mg->Add (gr1, "L");

Double_t $x 2$ [MAXREC], y2 [MAXREC], err_x2 [MAXREC], err_y2 [MAXREC];

int $i=0$; for (int $k=$ ArrowInfo.Pass [dataset-1] [pass-1]. Ind 1;

$\mathrm{k}<=$ ArrowInfo. Pass [dataset -1$]$ [pass -1$]$.Ind2; $\mathrm{k}++)\{$

arrow $=($ TArrow $*)$ Arrows. At $(k)$;

$\mathrm{x} 2[\mathrm{i}]=\operatorname{arrow}->\operatorname{GetX} 1() ; \mathrm{y} 2[\mathrm{i}]=\operatorname{GrossCounts}[\mathrm{k}] ; \operatorname{err} \mathrm{x} 2[\mathrm{i}]=0$.;

err_y2 $[i]=\operatorname{sqrt}($ GrossCounts $[\mathrm{k}]+$ BckgndData. MeanBackground);

i++; \}Int_t $n=$ ArrowInfo.Pass[dataset-1] [pass-1].Ind2

- ArrowInfo. Pass [dataset-1] [pass-1]. Ind1 +1;

TGraphErrors *gr2 = new TGraphErrors (n, x2, y2,err_x2, err_y2);

gr2->SetMarkerStyle(19+dataset); gr2->SetMarkerSize(1.);

gr2->SetMarkerColor(dataset); gr2->SetLineColor(dataset);

gr2->SetLineWidth (2); mg->Add(gr2,"P"); mg->Draw ("ALP");

mg->SetTitle(s); mg->GetXaxis()->SetLabelSize(0.05);

mg $\rightarrow$ GetYaxis ()->SetLabelSize(0.05); mg->GetXaxis()->SetTitleSize(0.06); 
mg->GetYaxis()->SetTitleSize(0.06);mg->GetXaxis()->SetTitle("Easting(m)"); mg->GetYaxis()->SetTitle("Counts"); mg->GetYaxis()->SetTitleOffset(1); \}//Mean of reconstructed source position with deviations //Position A Only

TCanvas *c2=new TCanvas ("cGC4", "Mean Northing vs Easting",600.,600.); c2->Divide $(2,2) ;$ TGraphErrors *gr $=$

new TGraphErrors (ArrowInfo. Ndataset, meanE, meanN, sdE, sdN); gr->SetTitle("Gross count RMS"); c2->cd(1);gPad->SetLeftMargin(0.15); gPad->SetBottomMargin(0.15); h->Draw();

TGraphErrors *gr1 = new TGraphErrors(1); $\operatorname{gr1->\operatorname {SetPoint}}(1, \operatorname{meanE}[0], \operatorname{meanN}[0]) ; \operatorname{gr} 1->\operatorname{SetPointError}(1, \operatorname{sdE}[0], \operatorname{sdN}[0])$; gr1->SetMarkerColor(1); gr1->SetLineColor(1); gr1->SetMarkerStyle (24);

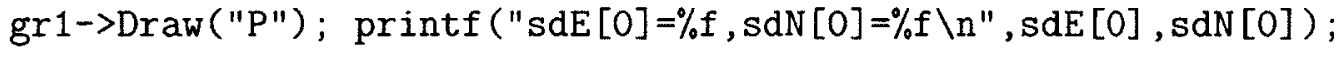

TGraphErrors *A_true; A_true = new TGraphErrors(1); A_true->SetPoint (1,456690-xoff , 1.682);A_true->SetPointError (1, 2, 2); A_true->SetMarkerSize(1);A_true->SetMarkerStyle (20); A_true->Draw ("P") ; printf ("sdE[0] =\%f, sdN [0] =\%f $\backslash \mathrm{n} ", \operatorname{sdE}[0], \operatorname{sdN}[0]$ ); TLegend* $\operatorname{leg} 2=$ new $\operatorname{TLegend}(0.9,0.67,0.64,0.90)$; leg2->SetHeader("2 m from road"); leg2->SetFillColor(0); leg2->SetBorderSize(1); leg2->AddEntry(A_true, "true position", "P"); leg2->AddEntry (gr1, "average measured", "P"); leg2->Draw(); 


\section{Appendix B}

\section{Directional Method}

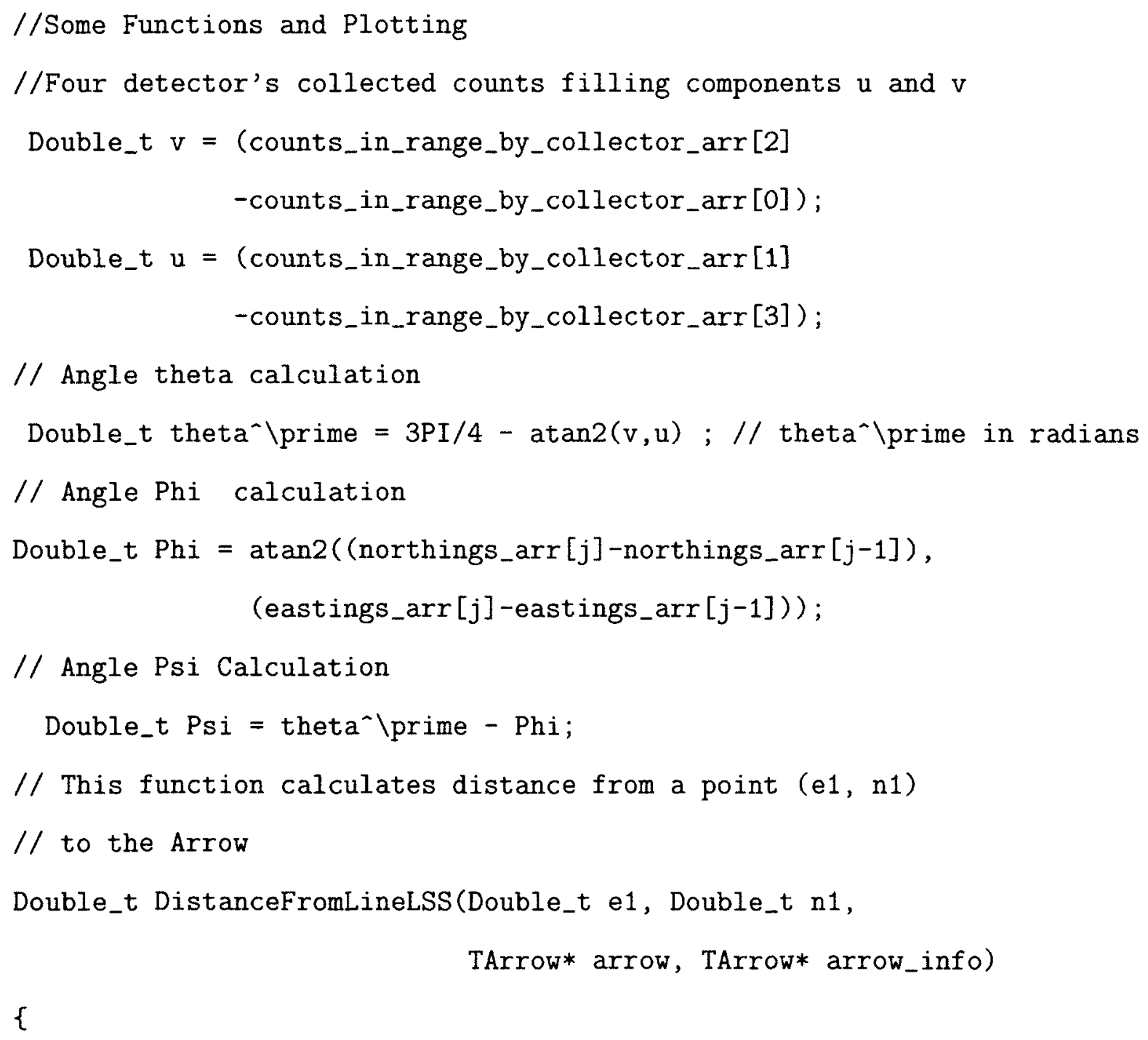




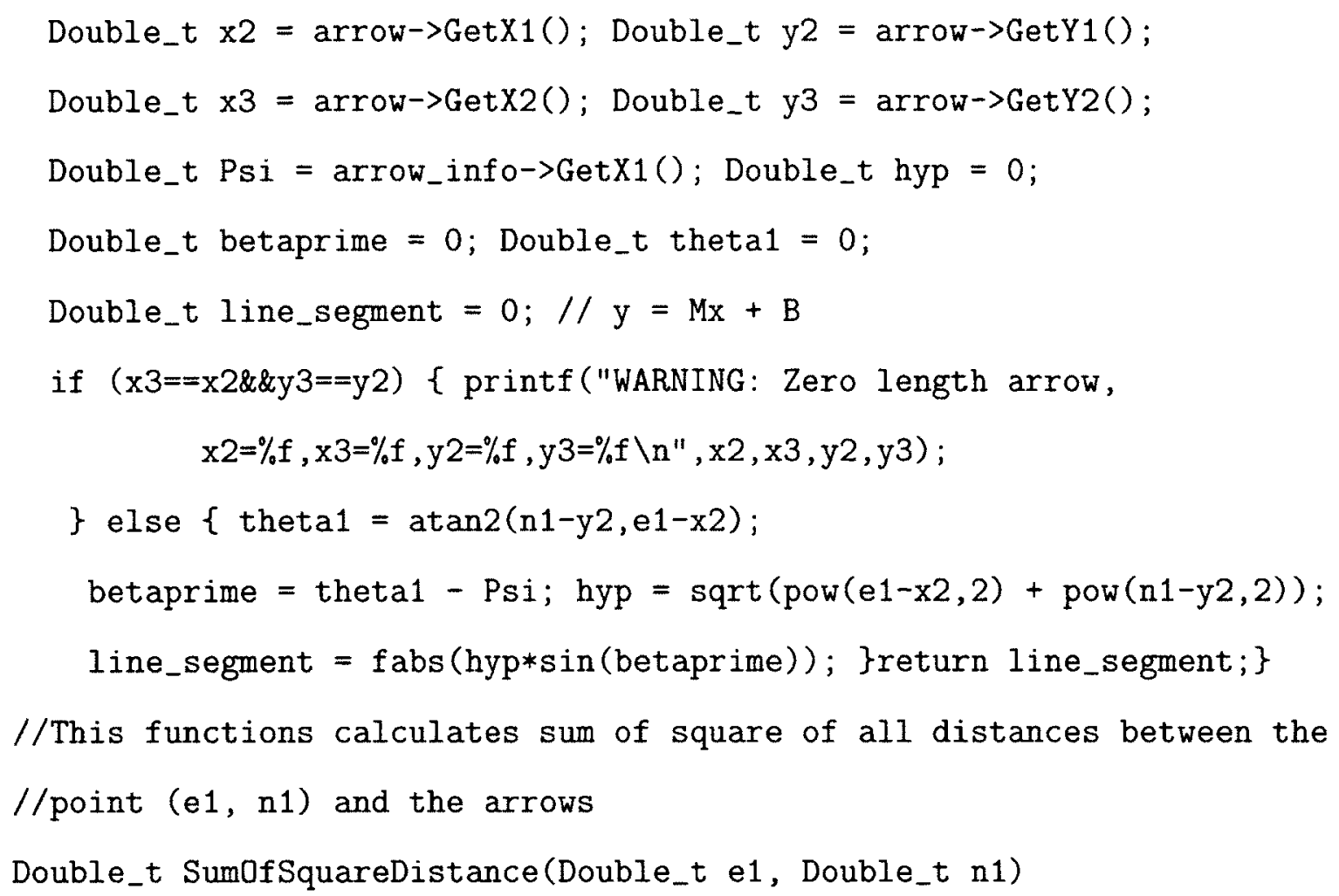


if (arrowlen>0\&\&dist $>0) \quad\{s s 2+=$ dist*dist; $\}$ \}return ss 2 ;

// This function draws all the arrows

void DrawData()\{ TArrow* arrow; Double_t* stats = ArrowStats () ;

gR00T->Reset (); TCanvas *c11=new TCanvas ("c11", "Data",600.,600.);

//c11->SetLeftMargin(0.14);TH2F* h; h = (TH2F *) gROOT->FindObject(" $h ")$;

if ( $h$ ) $h \rightarrow \operatorname{Delete}() ; h=$ new $\operatorname{TH} 2 F(" h ", " ", 100, x \min , x \max , 100$, ymin, ymax);

h->SetStats(false); $h->$ Draw () ; Double_t this_x=0; Double_t this_y=0;

for (int $j=0 ; j<$ NRecords $; j++)$ \{arrow $=($ TArrow $*$ ) Arrows $\cdot A t(j)$;

this_X $=\operatorname{arrow}->\operatorname{GetX1}()$; this_y $=\operatorname{arrow}->\operatorname{GetY} 1()$;

if (this_y>ymin \&\& this_y<ymax \&\& this_x>xmin \&\& this_ $x<x m a x)\{$ arrow->SetLineWidth(3); $\operatorname{arrow}->$ SetLineColor ((1));

arrow->SetFillColor (0); $\operatorname{arrow}->\operatorname{Draw}() ;\}\}$

h->GetXaxis ()->SetNdivisions (505); h->GetXaxis ()->SetNoExponent ();

h->GetYaxis()->SetNdivisions (505); h->GetYaxis()->SetNoExponent ();

h->GetXaxis()->SetLabelSize(0.05); h->GetYaxis()->SetLabelSize(0.05);

h->GetXaxis() $\rightarrow$ SetTitle ("Easting (m)");

h->GetXaxis()->SetTitleSize(0.06); h->GetXaxis()->SetTitle0ffset (0.85);

h->GetYaxis() $\rightarrow$ SetTitle ("Northing(m)");

h->GetYaxis () $\rightarrow$ SetTitleSize(0.06); h->GetYaxis ()->SetTitleOffset (0.85);

TGraph * GSource;

GSource = new TGraph (1); GSource->SetPoint (1, source [0], source [1]);

GSource->SetMarkerSize (1.2);GSource->SetMarkerStyle (20);

GSource->SetMarkerColor(3);GSource->SetFillColor(3);

GSource->Draw ("P");

$/ /$ This function draws 3D of the sum of square distance

void DrawSum0fSquares3D() \{Int_t nps $=20 ; / /$ sqrt of number of points

Double_t $\mathrm{x}, \mathrm{y}, \mathrm{z} ;$ Double_t* stats $=\operatorname{ArrowStats}()$; 


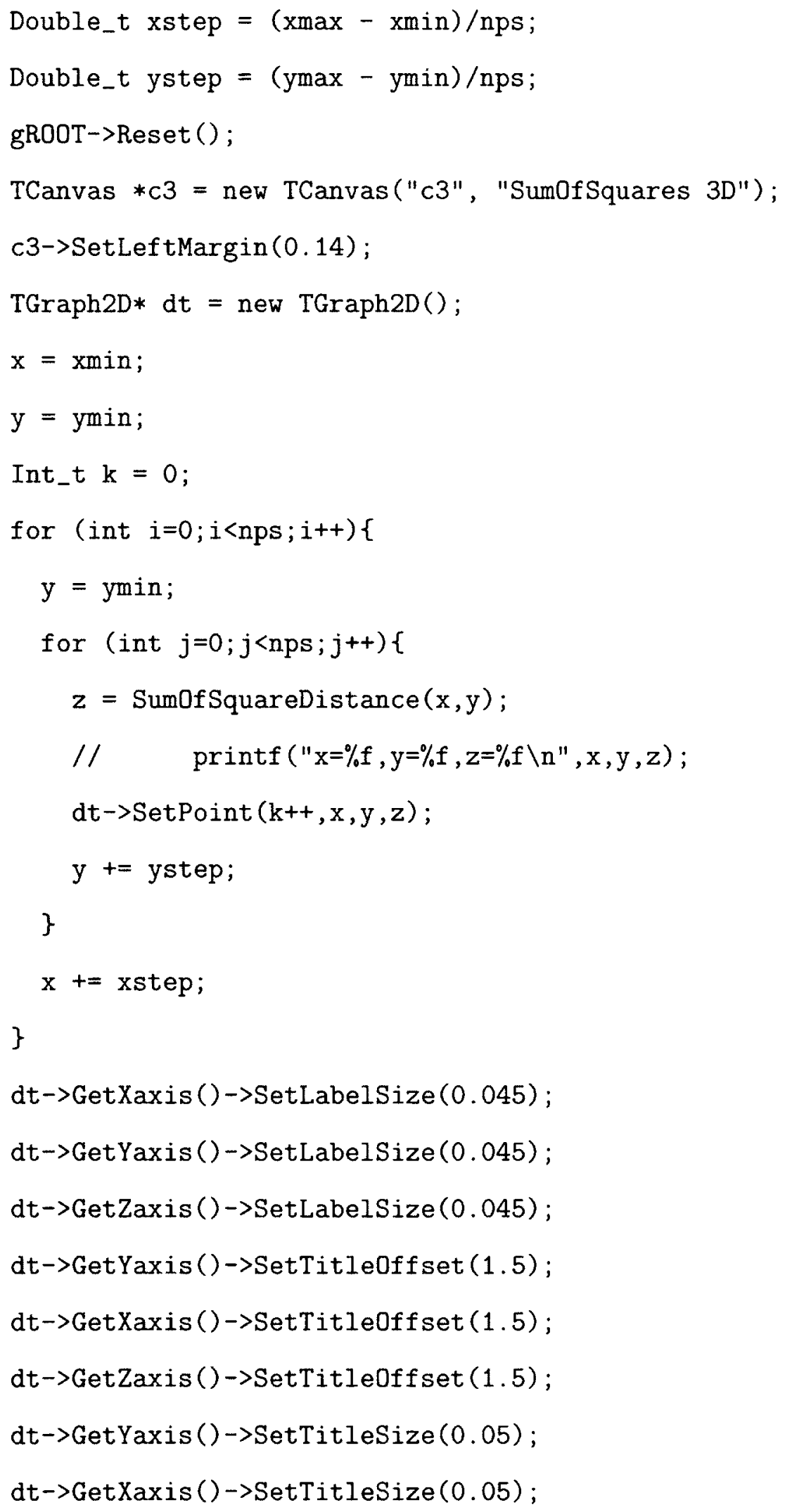




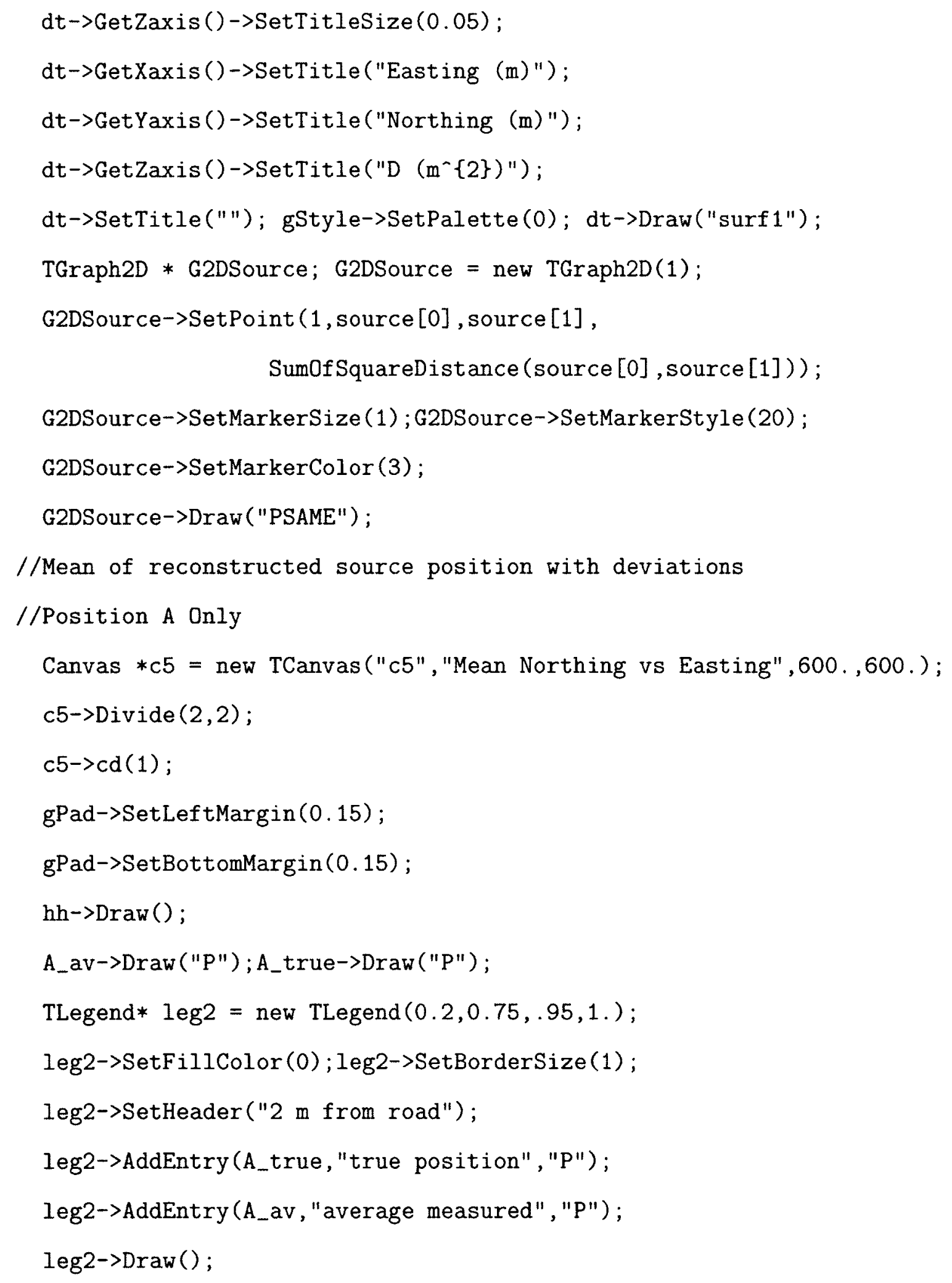




\section{References}

[1] International Atomic Energy Agency, Measures to strengthen international co-operation in nuclear, radiation and waste safety. The action plan for the safety of radiation sources and the security of radioactive material., Vienna IAEA Report GC/2000/34-GC(44)/7.

[2] Helle-Karina Aage and Uffe Korsbech, Handbook on Mobile Gamma-Ray Spectrometry, Technical University of Denmark, Lyngby, NT-65, Dec 2003 and revised (2004).

[3] International Atomic Energy Agency, Air-borne gamma ray spectrometer surveying, Vienna IAEA Technical reports Series 323.

[4] International Commision on Radiation Units and Measurements, Gamma-ray Spectrometry in the Environment, ICRU, Bethesda, Maryland, Report 53.

[5] F. James and M. Roos, Minuit: A System for Function Minimization and Analysis of the Parameter Errors and Correlations, Comput. Phys. Commun. 10 (1975) 343-367.

[6] Thomas Hjerpe and Christer Samuelsson, A Comparison between Gross and Net Count Methods when Searching for Orphan Radioactive Sources, Health Physics 84(2) (2003) 203-211.

[7] Thomas Hjerpe, Robert R. Finck and Christer Samuelsson, Statistical Data Evaluation in Mobile Gamma Spectrometry: An Optimization of on-line Search Strategies in the Scenario of Lost Point Sources, Health Physics 80(6) (2001) $563-70$.

[8] L. E. Sinclair, M. S. Khan, 2012 IEEE Symposium on Radiation Measurements and Applications, IEEE conference, City Center Marriott, Oakland, California, U.S.A. 
[9] G. F. Knoll, Radiation Detection and Measurement, 3rd edition, John Wiley \& Sons, Hoboken (2000).

[10] H. E. Johns and J. R. Cunningham, The Physics of Radiology. 4th edition. Charles C Thomas. Toronto (1983).

[11] The National Institute of Standards and Technology (NIST), XCOM: Photon Cross Sections Database; www.nist.gov/pml/data/xcom/ (2011).

[12] Doug Reilly and Norbert Enselin. Passive Nondestructive Assay of Nuclear Materials, United States Nuclear Regulatory Commission, US Government Printing Office-NUREG/CR-5550 LA-UR-90-732 (1991).

[13] Idaho National Laboratory, Gamma-Ray Spectrometry Center, US department of energy; http://www.inl.gov/gammaray/catalogs (1997).

[14] Karl H. Lieser, Nuclear and Radiochemistry: Fundamentals and Applications, John Wiley \& Sons, Weinheim (1997).

[15] G. Nelson and D. Reilly, Gamma-ray Interactions with matter, in Passive Nondestructive Analysis of Nuclear Materials, Los Alamos National Laboratory (1991).

[16] C. R. Nave, Department of Physics and Astronomy, Georgia State University; http://hyperphysis.phy-astr.gsu.edu/hbase/hph.html (2010).

[17] J. R. Clynch, Earth Coordinates, The University of New South Wales, Sydney, 2052.

[18] Minnesota Geospatial Information Office, Military Map Reading 201, http://www.mngeo.state.mn.us/.

[19] Demis website, An example of UTM zones over Europe, Rotterdamsweg 183C 2629 HD Delft, The Netherlands, http://www.demis.nl/../examples.

[20] MapTools website, Tools for plotting GPS coordinates, http://www.maptools.com/usingutm.

[21] Charles F. F. Karney, Transverse Mercator with an accuracy of a few nanometers, J. Geod 85 (2011) 475-485.

[22] William R. Leo, Techniques for Nuclear and Particle Physics Experiments, Springer, Berlin (1987). 
[23] Stephen A. Dyer. Survey of Instrumentation and Measurement. John Wiley and Sons, New York (2001).

[24] P. Martreau and R. Berndt, Handbook of Gamma Spectrometry Methods for non-destructive Assay of nuclear materials, EUR 19822 EN. Joint Research centre, Ispra-4th revised ed. (2010).

[25] R. B. Murray, IEEE Trans. Nucl. Sci. NS-22 (1975) 54.

[26] R. G. Kauf et al, IEEE Trans. Nucl. Sci. NS-17(3) (1970) 82.

[27] P. Zanzonico and Sherman Heller. The intraoperative gamma probe: Basic principles and choices available, Seminars in Nuclear medicine (2000) 33-48.

[28] Radiation Solutions Inc., Gamma-neutron User Manual, Mississauga (2008).

[29] Q. Bristow, The application of air-borne gamma-ray spectrometry in the search for radioactive debris from the Russian Satellite Cosmos 954, Current Research, Geological Survey of Canada Part B, Paper 78-1B (1978) 151-162.

[30] D. C. W Sanderson and J. M. Ferguson, The European capibility for environmental airborne gamma ray spectrometry, Radiat. Prot. Dosim. 73 (1997) 213-218.

[31] A. J. Cresswell and D. C. W Sanderson, The use of difference spectra with filtered rolling average background in mobile gamma spectrometry measurements, Nuclear Instruments and Methods in Physics Research A 607 (2009) 685-694.

[32] T. Hjerpe and C. Samuelsson, Shielded and unshielded geometries in the search for orphan sources, Applied Radiation and Isotopes 64 (2006) 551-555.

[33] P. Coll, Aspects Relating to the Calculation of doses in moving Radiation field., Health Physics 49(6) (1985) 1139-1146.

[34] M. Dowdall, M. A. Smethurst, R. Watson, A. Mauring, H. K. Aage, K. G. Anderson and S. E. Palsson, Car-borne gamma spectrometry: A virtual exercise in emergency response, Journal of Environmental RadioActivity 107 (2012) 68-77.

[35] Daniel J. Lingenfelter, Jeffrey A. Fessler, Clayton D. Scott and Zhong He, Benefits of Position-Sensitive Detectors for Source Detection with known background, IEEE Nuclear Science Symposium Conference Record N13 (2009) 126. 
[36] Carey L. Larsson. Harry Ing. Salah Djeffal. Hugh R. Andrews and Ming Zhang. Directional Gamma ray probe. US7470909 B2 United States Patent.

[37] J. Uher, C. Frojdh, J. Jakubek, S. Pospisil, G. Thungstrom and Z. Vykydal. Directional Radiation Detector, IEEE Nuclear Science Symposium Conference Record N24 (2007) 54.

[38] Audrey MacLeod, Patrick Boyle, David Hanna, Patrick Saull. Henry Seyward and Laurel Sinclair, All-scintillator Compton Gamma Imager, Physics in Canada, Vol. 65, No. 3.

[39] P. R. B. Saull, L. E. Sinclair, H. C. J. Seywerd, D. S. Hanna, P. J. Boyle and A. M. L. Macleod, First demonstration of a Compton gamma imager based on silicon photomultipliers, Nucl. Instr. Meth. A 679 (2012) 89-96.

[40] L. E. Sinclair, D. S. Hanna, A. M. L. Macleod and P. R. B. Saull, Simulations of a scintillator Compton Gamma Imager for Safety and Security, IEEE Trans. Nucl. Sci. 56, Iss 3 (2009) 1262-1268.

[41] Adam Wayne Lackie, A Directional Algorithm for the Electronically Collimated Radiation Detector- Master's Thesis, Louisiana State University and Agricultural and Mechanical College, Baton Rouge (2007).

[42] Google Inc. (2011), "Google Earth (version 5.1.3533.1731)", www.google.com/earth/index.html, 2011.

[43] Federal Aviation Administration, "Global Positioning System", Global Navigation Satellite Systems Group ; http://www.faa.gov/.../gnss/gps/, 2012. 